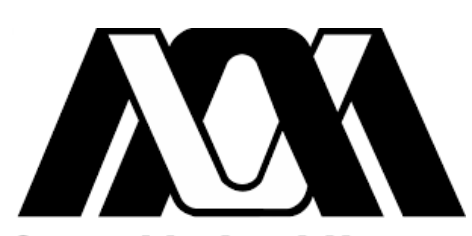

Casa abierta al tiempo

UNIVERSIDAD AUTÓNOMA METROPOLITANA - IZTAPALAPA DIVISIÓN DE CIENCIAS BÁSICAS E INGENIERÍA

\title{
ESTUDIO DE FAMILIAS DE SUCESIONES DE POLINOMIOS ORTOGONALES \\ UTILIZANDO MATRICES INFINITAS
}

\author{
Tesis que presenta \\ María Ivonne Arenas Herrera \\ Para obtener el grado de \\ Doctora en Ciencias Matemáticas
}

Director de tesis

\section{Dr. Luis Verde Star}

Sinodales:

Presidente: Dr. Carlos Enrique Signoret Poillon Secretario:

Vocal:

Vocal:

Vocal:
Dr. Roberto Quezada Batalla Dra. Martha Takane Imay Dr. Manuel Domínguez de la Iglesia Dr. Baltazar Aguirre Hernández
UAM-Iztapalapa UAM-Iztapalapa Instituto de Matemáticas UNAM Instituto de Matemáticas UNAM UAM-Iztapalapa 
En primer lugar quiero expresar mi infinito agradecimiento al director de esta tesis, Dr. Luis Verde Star, por aceptarme como su alumna de doctorado, por todos los conocimientos que me ha transmitido durante este proceso y por su amistad.

Agradezco a los sinodales que revisaron este trabajo de tesis: Dra. Martha Takane Imay, Dr. Manuel Domínguez de la Iglesia, Dr. Roberto Quezada Batalla, Dr. Baltazar Aguirre Hernández y Dr. Carlos Enrique Signoret Poillon, gracias por el tiempo dedicado, por sus comentarios y sugerencias aportadas.

Mi agradecimiento al Consejo Nacional de Ciencia y Tecnología (CONACyT), por otorgarme la beca y en general por el apoyo que brinda a la formación de capital humano científico y tecnológico.

Quiero agradecer de manera muy especial a Miguel González Vázquez por su amor incondicional, comprensión y apoyo durante estos nueve años juntos.

Finalmente, agradezco a mi madre María Emma y mis hermanos Carlos Antoni y Pablo Fernando por su amor y apoyo, siempre están en mi pensamiento. 


\section{Contenido}

Introducción $\quad$ VII

1 Caracterización y construcción de los polinomios ortogonales clásicos utilizando $\begin{array}{ll}\text { un enfoque matricial } & \mathbf{1}\end{array}$

1.1 Semi-matrices infinitas inferiores . . . . . . . . . . . . . . . . . . . . 1

1.2 Sucesiones de polinomios . . . . . . . . . . . . . . . . . 7

1.3 Sucesiones de polinomios ortogonales clásicos . . . . . . . . . . . . . . . 9

1.4 Construcción explícita de las matrices de polinomios ortogonales clásicos . . . . . . . 13

2 La clase de Hahn extendida de sucesiones de polinomios ortogonales discretos 17

2.1 Construcción explícita de matrices de polinomios ortogonales discretos . . . . . . 17

2.2 Ecuaciones en diferencias . . . . . . . . . . . . . . . . . . . . 20

3 La clase de Hahn extendida de sucesiones de polinomios $q$-ortogonales 25

3.1 Coeficientes de la relación de recurrencia de sucesiones de polinomios $q$-ortogonales . 25

3.2 Caracterización de la clase de Hahn $\mathcal{J}_{q} \ldots \ldots \ldots$. . . . . . . . . . . . 28

4 Polinomios ortogonales $q$-clásicos que tienen sucesiones simples de momentos 33

4.1 Sucesiones de polinomios ortogonales $q$-clásicos . . . . . . . . . . . . . . . 34

4.2 Familias de sucesiones $q$-ortogonales con sucesiones simples de momentos . . . . . . 36

4.3 Otras familias de polinomios $q$-ortogonales con momentos simples . . . . . . . . . . 40

5 Representación de matrices doblemente infinitas como series de Laurent no$\begin{array}{ll}\text { conmutativas } & 43\end{array}$

5.1 Matrices doblemente infinitas . . . . . . . . . . . . . . . . . . . . 44

5.2 Representación por diagonales y series de Laurent no-conmutativas . . . . . . . . . 48

5.3 Algunas propiedades del anillo $\mathfrak{L} \ldots \ldots \ldots \ldots$. . . . . . . . . . . . . . 52

$\begin{array}{lr}\text { Conclusiones } & 57\end{array}$

$\begin{array}{lr}\text { Referencias } & 59\end{array}$ 
La teoría de sucesiones de polinomios ortogonales constituye un tema clásico del análisis matemático y está relacionada con diversas e importantes ramas de la Matemática, como por ejemplo, las funciones especiales, las fracciones continuas, la interpolación y la aproximación, la integración numérica, la probabilidad, los métodos numéricos, la combinatoria y las ecuaciones diferenciales y en diferencias finitas. De hecho, su importancia dentro de la Física-Matemática comienza a finales del siglo XVIII, cuando se trataba de resolver problemas relativos a la mecánica celeste. La teoría de fracciones continuas da lugar al nacimiento de la teoría general sobre polinomios ortogonales. Quizás los trabajos de T. J. Stieltjes Jr. (1856-1894) sobre fracciones continuas permiten ver de una manera clara dicha relación con los polinomios ortogonales, ya que, Stieltjes en su famoso ensayo Recherches sur les fractions continues [29], desarrolló la teoría general de las $S$-fracciones, demostrando que bajo ciertas condiciones sobre sus parámetros, la sucesión de denominadores, $\left\{p_{n}\right\}_{n \geq 0}$, de dichas fracciones formaba una sucesión de polinomios ortogonales. Posteriormente el trabajo realizado por el matemático ruso P. L. Chebyshev, el cual estudió una gran variedad de problemas relacionados con los polinomios ortogonales, llegando a ellos al tratar de resolver ciertos problemas aplicados. Es así, que tanto a Stieltjes como a Chebyshev se les consideren los padres de la teoría de polinomios ortogonales que estaba por llegar a principios del siglo XX quedando consolidada en 1939 con la aparición de la monografía Orthogonal Polynomials de Gabor Szegö [30].

Dentro de la gran familia que forman los polinomios ortogonales, se encuentran los polinomios ortogonales clásicos (Hermite, Laguerre y Jacobi) los cuales satisfacen, según probó E. J. Routh [27] en 1885, una ecuación diferencial. Años más tarde S. Bochner [7] en 1929 probó lo mismo. Es bien conocido el hecho de que los teoremas de caracterización indican las principales propiedades que caracterizan a las familias clásicas de polinomios ortogonales. Una de las versiones más conocidas, y a su vez de las más antiguas, se debe a Sonin, la cual consiste en definir los polinomios clásicos como los únicos polinomios ortogonales que satisfacen la propiedad que sus derivadas, $\left\{p_{n}^{\prime}\right\}_{n \geq 0}$, también son ortogonales, demostrando que los polinomios de Jacobi, Laguerre y Hermite son clásicos.

Dicha propiedad, fue redescubierta por W. Hahn en 1935, quien también recuperó los polinomios de Bessel, no considerados por Sonin.

Es así como muchos ejemplos particulares de polinomios ortogonales aparecieron, en diferentes lugares y tiempos, al resolver problemas muy diversos y que se asocian con nombres como Stieltjes, Gauss, Legendre, Jacobi, Hermite, Bessel, Laguerre y Tchebyshev. La teoría general se desarrolló entre 1900 y 1935, pero el desarrollo de la computación generó alrededor de 1960 un nuevo interés en el tema, principalmente por las necesidades de los métodos numéricos y la teoría de aproximación. Desde entonces el estudio de los polinomios ortogonales, sus generalizaciones y sus aplicaciones, se ha realizado de manera continua por un grupo cada vez más numeroso de investigadores en diversas 
partes del mundo. Frecuentemente se realizan eventos internacionales sobre polinomios ortogonales y temas afines.

Una característica importante de las sucesiones de polinomios ortogonales se traduce en el hecho de que cada sucesión de polinomios ortogonales está determinada por una relación de recurrencia, llamada recurrencia de tres términos. Esto es equivalente a decir que cada sucesión de polinomios ortogonales mónicos queda determinada por un par de sucesiones de números $\left\{\beta_{k}: k \geq 0\right\} \mathrm{y}$ $\left\{\alpha_{k}: k \geq 0\right\}$, con la única condición de que $\alpha_{k} \neq 0$ para $k \geq 0$. Tales sucesiones son los coeficientes en la recurrencia de tres términos. Por lo tanto existe una infinidad de sucesiones de polinomios ortogonales. Pero no todas son relevantes o útiles. Por este motivo se estudian familias de sucesiones que satisfacen algunas condiciones adicionales que las hacen relevantes. El ejemplo más simple, como ya se mencionó anteriormente, es la familia de sucesiones $\left\{p_{k}(t): k \geq 0\right\}$ de polinomios ortogonales tales que la sucesión $\left\{p_{k}^{\prime}(t): k \geq 0\right\}$ de derivadas es también ortogonal. Esta familia incluye las conocidas sucesiones de Laguerre, Jacobi, Bessel y Hermite, que usualmente son llamadas sucesiones clásicas. Los polinomios de Legendre y Tchebyshev son casos especiales de los polinomios de Jacobi.

En la literatura, un problema general en la teoría de polinomios ortogonales es la caracterización de familias que satisfacen algunas condiciones adicionales, por ejemplo, una ecuación diferencial o en diferencias finitas. Este problema se comenzó a estudiar alrededor de 1925, por Bochner, Hahn y Favard, y continúa siendo de interés para muchos investigadores.

Debido al origen de los polinomios ortogonales dentro del análisis matemático, la teoría se ha desarrollado utilizando herramientas analíticas, y los aspectos algebraicos del tema casi no se han estudiado. Recientemente L. Verde mostró en [34] que una parte considerable de la teoría general se puede obtener usando herramientas puramente algebraicas del álgebra lineal en el contexto de una álgebra de matrices infinitas. En ese trabajo se caracterizan las sucesiones clásicas y se encuentra una fórmula explícita para los coeficientes de la relación de recurrencia de todas las sucesiones clásicas. En el artículo [36] se extiende ese trabajo para incluir a las familias de polinomios ortogonales discretos y los $q$-ortogonales.

La organización de esta Tesis es la siguiente:

\section{Capítulo 1}

Se introduce el concepto de semi-matriz infinita inferior, y se define el índice para estas matrices infinitas $A$ así como propiedades de éste. Se define el conjunto de las matrices semi-inferiores y se dan ejemplos explícitos de matrices en dicho conjunto, así como elementos particulares y características en este conjunto que serán usados con frecuencia. Se introduce el conjunto de matrices triangulares inferiores invertibles y posteriormente las matrices banda y sus propiedades; así como también se habla de las matrices de Toeplitz, matrices mónicas y matrices diagonalmente similares. Mediante la base de potencias se expresan sucesiones de polinomios con coeficientes reales construyendo una matriz cuyas entradas son los coeficientes de los polinomios de la sucesión. Se demuestra que una sucesión de polinomios mónicos cuya matriz asociada $A$ es ortogonal con respecto a algún funcional lineal si y sólo si la matriz $L$ es una matriz en banda. Además se obtiene una relación de recurrencia a tres términos igualando las $k$-ésimas filas de una ecuación matricial y se obtienen fórmulas explícitas para los coeficientes de la relación de recurrencia.

Se introduce el concepto de matriz de polinomios ortogonales (MPO) mónicos y se demuestra cuándo una $M P O$ cumple con ser clásica, se caracterizan las $M P O$ clásicas por medio de un par de sucesiones que corresponden a los coeficientes de la relación de recurrencia a tres términos. 
Finalmente, se consideran familias de polinomios relativamente simples obtenidas al dar ciertos valores a los parámetros relacionados con la relación de recurrencia.

\section{Capítulo 2}

Este Capítulo está dedicado al estudio de ciertas familias de polinomios ortogonales discretos. Se comienza definiendo el operador en diferencias $\Delta_{w}$ actuando sobre polinomios con coeficientes complejos, utilizando la base de Newton se construye la representación matricial de dicho operador así como la matriz de representación del operador multiplicación, se define la clase de Hahn y se caracterizan sus elementos en términos de sus matrices de coeficientes de la relación de recurrencia. Posteriormente se demuestra que el conjunto de matrices de la clase de Hahn satisfacen una ecuación matricial caracterizada por una matriz diagonal y polinomios $h$ y $f$, de grados uno y a lo más dos, respectivamente. A continuación se obtienen fórmulas explícitas tanto para los elementos de dicha matriz diagonal cuyos elementos son llamados los eigenvalores, así como para los coeficientes de los polinomios $h$ y $f$. Se demuestra el usualmente llamado teorema de Hahn.

\section{Capítulo 3}

El Capítulo 3 contiene fórmulas explícitas para los coeficientes de la relación de recurrencia de las sucesiones de polinomios $q$-ortogonales de la clase de Hahn extendida. Se define la actuación del operador $q$-derivada, $\mathcal{D}_{q}$, sobre el conjunto de los polinomios ortogonales con coeficientes complejos. Se introduce la matriz de representación de dicho operador, $D_{q}$, y se define la clase de Hahn para éste. Se incluye un resultado que recoge la caracterización de los polinomios $q$-ortogonales, al construir una matriz $E_{q}$ de representación con respecto a la base canónica de monomios de la función $p(x) \rightarrow p(q x)$, obteniendo que una matriz $A$ se encuentra en la clase de Hahn extendida si satisface una ecuación matricial de la forma $Y A E_{q}=A B$ para alguna matriz $B=D_{q}\left(h(X)+D_{q} f(X)\right)$, donde $h$ y $f$ son polinomios de grados uno y a lo más dos, respectivamente.

\section{Capítulo 4}

En el Capítulo 4 se estudian las sucesiones de momentos de polinomios ortogonales $q$-clásicos obteniendo una relación de recurrencia de tres términos con coeficientes variables que dependen de los coeficientes de la ecuación en $q$-diferencias satisfecha por los polinomios y los resultados alcanzados aparecen en [4]. Considerando los casos en los cuales la relación de recurrencia se simplifica se obtienen algunas familias de polinomios ortogonales $q$-clásicos que tienen fórmulas explícitas simples para los momentos, expresadas en términos de los parámetros en la ecuación en $q$-diferencias. Se comienza definiendo una $(0,2)$-matriz banda $C$, y se visualizan algunos de sus elementos, se introduce la matriz diagonal de índice cero $\Lambda$, y se dice que una sucesión de polinomios $q$-clásicos es ortogonal si la matriz $A$ asociada a ellos, satisface una ecuación matricial de la forma $A C=\Lambda A$, para algún par de polinomios $h$ y $g$. Con ayuda de la matriz $C$ se demuestra que la sucesión de momentos $\mu_{k}$ satisface una relación de recurrencia a tres términos.

Se construyen varios ejemplos de familias de polinomios ortogonales $q$-clásicos que tienen sucesiones simples de momentos. Con ayuda de cinco parámetros se encuentran fórmulas explícitas para los momentos y los coeficientes de la relación de recurrencia. Se observa que dando un valor particular a ciertos parámetros la matriz $C$, se convierte en bidiagonal y entonces esto permite obtener una relación de recurrencia a dos términos. Así, dando algunos valores apropiados a los parámetros se obtienen familias de polinomios $q$-ortogonales tales como: los polinomios $q$-Krawtchouk no estándar, 
que no han sido considerados en la literatura de polinomios ortogonales, los polinomios $q$-Bessel, los polinomios $q$-Laguerre/Wall pequeños y los polinomios $q$-Jacobi grandes.

Finalmente, se estudian familias de polinomios ortogonales que satisfacen la ecuación en $q$-diferencias donde los coeficientes de los términos constantes de dos polinomios $h$ y $f$ son iguales a cero, obteniendo que todos los momentos con $n$ impar son iguales a cero, llevando a obtener familias de polinomios q-ortogonales con momentos muy simples.

\section{Capítulo 5}

En el Capítulo 5 se extiende el estudio de las matrices infinitas al estudio de matrices doblemente infinitas, donde los índices de los elementos de éstas corren sobre el conjunto de los números enteros, los resultados alcanzados aparecen en [5]. Se comienza presentando el conjunto de matrices doblemente infinitas, $\mathfrak{L}$, y se mencionan propiedades y características. Se definen dos conjuntos: un conjunto de matrices diagonales de índice $m, \mathcal{D}_{m}$, y el segundo, un conjunto de vectores columna infinitos, $\mathcal{V}$, y se demuestra que los elementos de $\mathfrak{L}$ están conectados con series de Laurent. Se presenta la multiplicación de matrices doblemente infinitas, y se habla de una traslación por $m$ posiciones en dirección diagonal de los elementos de matrices bi-infinitas, y se define a $A^{[m]}$ como el desplazamiento diagonal de $A$ por $m$ pasos haciendo uso de matrices de desplazamiento $S$. Se enumeran algunas propiedades básicas del conjunto de matrices doblemente infinitas.

Se introduce una representación por diagonales de los elementos de $\mathfrak{L}$, la cual parece una serie de Laurent no-conmutativa, se encuentra una expresión para la multiplicación en $\mathfrak{L}$ usando la representación por diagonales. Se demuestra una propiedad que permite hablar sobre la invertibilidad de los elementos del conjunto de matrices doblemente infinitas.

Usando la representación diagonal de los elementos de $\mathfrak{L}$, se demuestran teoremas con algunas propiedades relacionadas con similaridad y conmutatividad. Se prueba que las matrices bi-infinitas también se pueden expresar como una serie de Laurent con un desplazamiento ponderado en lugar de usar matrices de desplazamiento $S$. Por último, se definen dos tipos de derivadas para matrices doblemente infinitas.

La presente Tesis ha sido escrita en LATEX. Cada uno de los Capítulos de la Tesis comienza con una introducción donde se describe el contenido del mismo. Se dividen éstos en secciones, que a su vez pueden contener subsecciones. Las referencias bibliográficas, preparadas con BibTEX aparecen citadas en orden alfabético de acuerdo al idioma castellano. 
CAPÍTULO 1

\section{CARACTERIZACIÓN Y CONSTRUCCIÓN DE LOS POLINOMIOS ORTOGONALES CLÁSICOS UTILIZANDO UN ENFOQUE MATRICIAL}

Debido al origen de los polinomios ortogonales dentro del Análisis Matemático, la teoría se ha desarrollado utilizando herramientas analíticas, y los aspectos algebraicos del tema casi no se han estudiado. En este capítulo, usando un enfoque matricial se estudian las sucesiones de polinomios ortogonales, es decir, usando herramientas puramente algebraicas del álgebra lineal en el contexto de un álgebra de matrices infinitas llamadas semi-matrices inferiores, se puede obtener una parte considerable de la teoría general de los polinomios ortogonales. Se quiere mostrar que el enfoque matricial produce resultados generales sobre sucesiones de polinomios. No se quiere reconstruir toda la teoría de polinomios ortogonales, sólo queremos ocuparnos de la caracterización y construcción de polinomios ortogonales clásicos. Se relacionarán matrices con sucesiones de polinomios y se caracterizarán las sucesiones ortogonales en términos de matrices. Se obtendrán fórmulas explícitas para los coeficientes de recurrencia de todas las sucesiones clásicas.

\subsection{Semi-matrices infinitas inferiores}

Se consideran matrices infinitas $A=\left[a_{j, k}\right]$ donde los índices corren en los enteros no negativos y las entradas son números reales.

Definición 1.1 Se dice que $A$ es una semi-matriz inferior si existe un entero $m$ tal que $a_{j, k}=0$ siempre que $j-k<m$. Se denota por $\mathcal{L}$ el conjunto de las semi-matrices inferiores.

Se dice que la entrada $a_{j, k}$ está en la $n$-ésima diagonal de $A$ si $j-k=n$. Además, si $m>n$ entonces la $m$-ésima diagonal se encuentra por debajo de (a la izquierda de) la $n$-diagonal. Se dice que un elemento distinto de cero en $\mathcal{L}$ es una matriz diagonal si todo elemento distinto de cero se encuentra en una sola diagonal. Se tiene que $\mathcal{L}$ es un espacio vectorial real con la adición natural de matrices y multiplicación por escalares, además es cerrado bajo la multiplicación de matrices.

Definición 1.2 Sea $A \in \mathcal{L}$ distinta de cero y sea $m$ el entero mínimo tal que $A$ tiene al menos una entrada distinta de cero en la $m$-ésima diagonal. Entonces se dice que $A$ tiene índice $m$ y se denota por ind $(A)=m$. Se define el índice de la matriz cero como infinito. 
Sean $A, B \in \mathcal{L}$, con $\operatorname{ind}(A)=m$ y $\operatorname{ind}(B)=n$. Entonces el producto de $C=A B$ es un elemento bien definido de $\mathcal{L}$ y

$$
c_{i, k}=\sum_{j=k+n}^{i-m} a_{i, j} b_{j, k}, \quad i-k \geq m+n .
$$

Además el $\operatorname{ind}(A B) \geq m+n$. Si $A \in \mathcal{L}$ entonces una condición suficiente (pero no necesaria) para que $A$ tenga una inversa bilateral (funciona como inversa por la derecha e inversa por la izquierda) es que $\operatorname{ind}(A)=0$ y $a_{k, k} \neq 0$ para $k \geq 0$. En algunos de los ejemplos siguientes se representan las semi-matrices infinitas escribiendo solamente una sección finita de ellas, para simplificar la escritura.

EJEMPLO 1.1 La siguiente matriz $A$ es una semi-matriz inferior infinita donde $\operatorname{ind}(A)=0, j-k<$ 0 , y cuenta con las diagonales $0,1,2,3,4, \ldots$, esto es:

$$
A=\left(\begin{array}{ccccc}
a_{00} & 0 & 0 & 0 & 0 \\
a_{10} & a_{11} & 0 & 0 & 0 \\
a_{20} & a_{21} & a_{22} & 0 & 0 \\
a_{30} & a_{31} & a_{32} & a_{33} & 0 \\
a_{40} & a_{41} & a_{42} & a_{43} & a_{44}
\end{array}\right) .
$$

Ejemplo 1.2 Otra semi-matriz inferior es la siguiente matriz $B$, donde $\operatorname{ind}(B)=-1, j-k<-1$ y cuenta con las diagonales $-1,0,1,2,3, \ldots$, es decir, $B$ tiene la siguiente forma:

$$
B=\left(\begin{array}{cccc}
b_{00} & b_{01} & 0 & 0 \\
b_{10} & b_{11} & b_{12} & 0 \\
b_{20} & b_{21} & b_{22} & b_{23} \\
b_{30} & b_{31} & b_{32} & b_{33}
\end{array}\right)
$$

Denotando por $\mathcal{G}$ al conjunto de matrices triangulares inferiores invertibles, donde $\mathcal{G}$ es un grupo bajo la multiplicación de matrices, la unidad es la matriz identidad $I$ cuyas entradas en la diagonal 0 (diagonal principal) son igual a 1 y todo el resto de las entradas es cero. Se definen a continuación algunos elementos particulares de $\mathcal{L}$ que serán usados frecuentemente.

Sea $X$ la matriz diagonal de índice -1 con $x_{j, j+1}=1$ para $j \geq 0$. Denotando por $\hat{X}$ la matriz transpuesta de $X$. Se observa que $\hat{X}$ es diagonal con índice igual a 1 . Nótese que $X \hat{X}=I$ y $\hat{X} X=J$, donde $J$ es la matriz diagonal de índice cero cuya entrada en la posición $(0,0)$ es igual a cero, y sus entradas en las posiciones $(j, j)$ igual a 1 para $j \geq 1$. Es decir, $J$ es diferente de la matriz identidad $I$ en la posición $(0,0)$. Además, $\hat{X}$ es una matriz inversa derecha para $X$ pero no una matriz inversa izquierda.

EJemplo 1.3 Sean $X=\left(\begin{array}{llll}0 & 1 & 0 & 0 \\ 0 & 0 & 1 & 0 \\ 0 & 0 & 0 & 1\end{array}\right), y \quad \hat{X}=\left(\begin{array}{lll}0 & 0 & 0 \\ 1 & 0 & 0 \\ 0 & 1 & 0 \\ 0 & 0 & 1\end{array}\right)$, entonces
$X \hat{X}=\left(\begin{array}{lll}1 & 0 & 0 \\ 0 & 1 & 0 \\ 0 & 0 & 1\end{array}\right)=I, \quad \widehat{X} X=\left(\begin{array}{llll}0 & 0 & 0 & 0 \\ 0 & 1 & 0 & 0 \\ 0 & 0 & 1 & 0 \\ 0 & 0 & 0 & 1\end{array}\right)=J$. 
Si $m$ es un entero positivo entonces $X^{m}$ es la matriz diagonal de indice $-m$ con todas sus entradas en dicha diagonal iguales a 1 . Análogamente, $\hat{X}^{m}$ es diagonal de indice $m$ y todas sus entradas en la $m$-ésima diagonal son iguales a 1 .

EJEMPLO 1.4 Sea $X=\left(\begin{array}{lll}0 & 1 & 0 \\ 0 & 0 & 1 \\ 0 & 0 & 0\end{array}\right)$ entonces

$$
X^{2}=\left(\begin{array}{lll}
0 & 1 & 0 \\
0 & 0 & 1 \\
0 & 0 & 0
\end{array}\right)\left(\begin{array}{lll}
0 & 1 & 0 \\
0 & 0 & 1 \\
0 & 0 & 0
\end{array}\right)=\left(\begin{array}{lll}
0 & 0 & 1 \\
0 & 0 & 0 \\
0 & 0 & 0
\end{array}\right),
$$

como se puede observar, se tiene una matriz diagonal donde $\operatorname{ind}\left(X^{2}\right)=-2$. Luego, el índice de $\hat{X}^{2}$ es 2 .

$$
\hat{X}^{2}=\left(\begin{array}{lll}
0 & 0 & 0 \\
1 & 0 & 0 \\
0 & 1 & 0
\end{array}\right)\left(\begin{array}{lll}
0 & 0 & 0 \\
1 & 0 & 0 \\
0 & 1 & 0
\end{array}\right)=\left(\begin{array}{lll}
0 & 0 & 0 \\
0 & 0 & 0 \\
1 & 0 & 0
\end{array}\right) .
$$

Por otro lado, se denota por $D$ la matriz de indice 1 con entradas $d_{j, j-1}=j$ para $j \geq 1$. Denotando por $\hat{D}$ la matriz diagonal de indice -1 con entradas $\hat{d}_{j-1, j}=1 / j$ para $j \geq 1$. Es decir,

$$
D=\left(\begin{array}{cccccc}
0 & 0 & 0 & 0 & 0 & \ldots \\
1 & 0 & 0 & 0 & 0 & \ldots \\
0 & 2 & 0 & 0 & 0 & \ldots \\
0 & 0 & 3 & 0 & 0 & \ldots \\
0 & 0 & 0 & 4 & 0 & \ldots \\
\vdots & & & & \ddots &
\end{array}\right), \quad \hat{D}=\left(\begin{array}{cccccc}
0 & 1 & 0 & 0 & 0 & \ldots \\
0 & 0 & 1 / 2 & 0 & 0 & \ldots \\
0 & 0 & 0 & 1 / 3 & 0 & \ldots \\
0 & 0 & 0 & 0 & 1 / 4 & \\
0 & 0 & 0 & 0 & 0 & \ddots \\
\vdots & & & & & \ddots
\end{array}\right)
$$

Es fácil ver que $\hat{D} D=I, D \hat{D}=J$. Otras identidades útiles son $X D-D X=I, J \hat{X}=\hat{X}, J D=D$, $\hat{D} J=\hat{D}$ y $X J=X$.

Sea $A \in \mathcal{G}$. Un simple cálculo muestra que $X A^{-1} \hat{X}$ es inversa bilateral (funciona como inversa derecha e inversa izquierda) de $X A \hat{X}$ y también que $\hat{D} A^{-1} D$ es inversa bilateral de $\hat{D} A D$.

Obsérvese que $X A \hat{X}$ se obtiene de $A$, es decir, por desplazamiento de ésta una posición a la izquierda y después por desplazar una posición hacia arriba, de manera que la 0-ésima fila y columna desaparecen.

Es importante resaltar el efecto de multiplicar un elemento de $\mathcal{L}$ por alguna de las matrices $X$ o $\hat{X}$, esta multiplicación puede ser por la derecha o izquierda. Se quiere decir lo siguiente, sea $B \in \mathcal{L}$ de la forma

$$
B=\left(\begin{array}{cccc}
b_{00} & b_{01} & 0 & 0 \\
b_{10} & b_{11} & b_{12} & 0 \\
b_{20} & b_{21} & b_{22} & b_{23} \\
b_{30} & b_{31} & b_{32} & b_{33}
\end{array}\right),
$$

el efecto de multiplicar $B$ por $X$ a la derecha, es que ésta traslada la matriz $B$ una columna a la derecha, y el efecto de multiplicar $B$ por $X$ a la izquierda es trasladar la matriz $B$ una fila hacia 
arriba, esto es,

$$
\begin{aligned}
& B X=\left(\begin{array}{cccc}
b_{00} & b_{01} & 0 & 0 \\
b_{10} & b_{11} & b_{12} & 0 \\
b_{20} & b_{21} & b_{22} & b_{23} \\
b_{30} & b_{31} & b_{32} & b_{33}
\end{array}\right)\left(\begin{array}{cccc}
0 & 1 & 0 & 0 \\
0 & 0 & 1 & 0 \\
0 & 0 & 0 & 1 \\
0 & 0 & 0 & 0
\end{array}\right)=\left(\begin{array}{cccc}
0 & b_{00} & b_{01} & 0 \\
0 & b_{10} & b_{11} & b_{12} \\
0 & b_{20} & b_{21} & b_{22} \\
0 & b_{30} & b_{31} & b_{32}
\end{array}\right) \\
& X B=\left(\begin{array}{cccc}
0 & 1 & 0 & 0 \\
0 & 0 & 1 & 0 \\
0 & 0 & 0 & 1 \\
0 & 0 & 0 & 0
\end{array}\right)\left(\begin{array}{cccc}
b_{00} & b_{01} & 0 & 0 \\
b_{10} & b_{11} & b_{12} & 0 \\
b_{20} & b_{21} & b_{22} & b_{23} \\
b_{30} & b_{31} & b_{32} & b_{33}
\end{array}\right)=\left(\begin{array}{cccc}
b_{10} & b_{11} & b_{12} & 0 \\
b_{20} & b_{21} & b_{22} & b_{23} \\
b_{30} & b_{31} & b_{32} & b_{33} \\
b_{40} & b_{41} & b_{42} & b_{43}
\end{array}\right) \text {. }
\end{aligned}
$$

Por otro lado, el efecto de multiplicar un elemento de $\mathcal{L}$ por la matriz $\hat{X}$ es el siguiente, multiplicar $B$ por $\hat{X}$ a la derecha, traslada una columna a la izquierda las entradas de $B$; y el efecto de multiplicar $B$ por $\hat{X}$ a la izquierda traslada una fila hacia abajo las entradas de $B$, es decir,

$$
\begin{aligned}
& B \hat{X}=\left(\begin{array}{cccc}
b_{00} & b_{01} & 0 & 0 \\
b_{10} & b_{11} & b_{12} & 0 \\
b_{20} & b_{21} & b_{22} & b_{23} \\
b_{30} & b_{31} & b_{32} & b_{33}
\end{array}\right)\left(\begin{array}{cccc}
0 & 0 & 0 & 0 \\
1 & 0 & 0 & 0 \\
0 & 1 & 0 & 0 \\
0 & 0 & 1 & 0
\end{array}\right)=\left(\begin{array}{cccc}
b_{01} & 0 & 0 & 0 \\
b_{11} & b_{12} & 0 & 0 \\
b_{21} & b_{22} & b_{23} & 0 \\
b_{31} & b_{32} & b_{33} & 0
\end{array}\right) \\
& \hat{X} B=\left(\begin{array}{cccc}
0 & 0 & 0 & 0 \\
1 & 0 & 0 & 0 \\
0 & 1 & 0 & 0 \\
0 & 0 & 1 & 0
\end{array}\right)\left(\begin{array}{cccc}
b_{00} & b_{01} & 0 & 0 \\
b_{10} & b_{11} & b_{12} & 0 \\
b_{20} & b_{21} & b_{22} & b_{23} \\
b_{30} & b_{31} & b_{32} & b_{33}
\end{array}\right)=\left(\begin{array}{cccc}
0 & 0 & 0 & 0 \\
b_{00} & b_{01} & 0 & 0 \\
b_{10} & b_{11} & b_{12} & 0 \\
b_{20} & b_{21} & b_{22} & b_{23}
\end{array}\right) .
\end{aligned}
$$

Las matrices $D$ y $\hat{D}$ producen los mismos efectos que las matrices $\hat{X}$ y $X$, respectivamente, claro salvo unas constantes. Entonces el efecto de multiplicar un elemento de $\mathcal{L}$ por éstas, es el mismo que producen $X$ y $\hat{X}$, es decir, se tienen traslaciones por filas y columnas hacia arriba, abajo, derecha o izquierda.

Lema 1.3 Sea $A \in \mathcal{G}$. Entonces $A^{-1} J A D=D$.

Demostración. Véase que $A^{-1} J A$ es diferente de $J$ sólo en la 0-ésima columna y entonces $A^{-1} J A D$ es el producto de las matrices

$$
\left(\begin{array}{ccccc}
0 & 0 & 0 & 0 & \ldots \\
* & 1 & 0 & 0 & \ldots \\
* & 0 & 1 & 0 & \ldots \\
* & 0 & 0 & 1 & \ldots \\
\vdots & & & & \ddots
\end{array}\right)\left(\begin{array}{ccccc}
0 & 0 & 0 & 0 & \ldots \\
1 & 0 & 0 & 0 & \ldots \\
0 & 2 & 0 & 0 & \ldots \\
0 & 0 & 3 & 0 & \ldots \\
\vdots & & & & \ddots
\end{array}\right)
$$

lo cual es igual a $D$.

Definición 1.4 Se dice que $A \in \mathcal{L}$ está en banda si existe un par de enteros $(m, n)$ tal que $n \leq m$ y todas las entradas distintas de cero de $A$ se encuentran entre las diagonales de índices $n$ y $m$. En tal caso se dice que $A$ es una $(n, m)$-matriz banda. 
Conviene destacar que el conjunto de matrices banda es cerrado bajo la multiplicación y adición, pero una matriz banda puede tener una matriz inversa que no es matriz banda. La transpuesta de una $(n, m)$-matriz banda es una $(-m,-n)$-matriz banda.

Por otro lado, el conjunto de las semi-matrices inferiores $\mathcal{L}$ contiene a las matrices de la forma

$$
T=\sum_{k=0}^{\infty} c_{k} \hat{X}^{k}+\sum_{k=1}^{m} c_{-k} X^{k}=\left(\begin{array}{ccccc}
c_{0} & c_{-1} & c_{-2} & c_{-3} & \cdots \\
c_{1} & c_{0} & c_{-1} & c_{-2} & \cdots \\
c_{2} & c_{1} & c_{0} & c_{-1} & \cdots \\
c_{3} & c_{2} & c_{1} & c_{0} & \cdots \\
\vdots & \vdots & \vdots & \vdots & \ddots
\end{array}\right),
$$

donde los coeficientes $c_{j}$ son reales y $m$ es un entero positivo, a estas matrices se les conoce como matrices de Toeplitz. Nótese que, si $c_{-m} \neq 0$ entonces $i n d(T)=-m$. La 0-ésima columna y la 0-ésima fila de $T$ son respectivamente,

$$
\left(c_{0}, c_{1}, c_{2}, \ldots, c_{n}, \ldots\right)^{t}, \quad \text { y } \quad\left(c_{0}, c_{-1}, c_{-2}, \ldots, c_{-m}, 0,0, \ldots,\right) .
$$

Proposición 1.5 Sea $A \in \mathcal{L}$ con ind $(A)=n$ tal que $A X=X A$. Si $n \leq 0$ entonces $A$ es un polinomio en $X$ de grado $-n$, y si $n \geq 1$ entonces $A=0$.

Demostración. Como $X \hat{X}=I$ la ecuación $A X=X A$ produce

$$
A=X A \hat{X}
$$

entonces vemos que $A$ debe ser una matriz de Toeplitz.

La 0-ésima columna de $X A$ es $\left(a_{10}, a_{20}, a_{30}, \ldots,\right)^{t}$. Ésta es la 0-ésima columna de $A$ desplazada un lugar hacia arriba. La 0-ésima columna de $A X$ tiene entradas cero. Por lo tanto, $a_{j, 0}=0$ para $j \geq 1$, y como $A$ es Toeplitz, todas las entradas de $A$ abajo de la diagonal de índice cero deben ser cero. Por tanto, si $n>0$ entonces $A=0$ y si $n \leq 0$ se debe tener

$$
A=\sum_{k=0}^{-n} a_{0, k} X^{k} .
$$

Corolario 1.6 Sea $L=B^{-1} X B$ para algún $B \in \mathcal{G}$. Sea $A \in \mathcal{L}$ tal que $A L=L A$. Entonces, si $\operatorname{ind}(A)=n \leq 0$ entonces $A$ es un polinomio en $L$ de grado $-n$, y si $\operatorname{ind}(A) \geq 1$ entonces $A=0$.

Demostración. De $A L=L A$ y $L=B^{-1} X B$ se obtiene que $B A B^{-1}$ conmuta con $X$, por la Proposición 1.5 ésta es cero cuando $n>0$, y entonces $A=0$. Si $n \leq 0$ la proposición da como resultado $B A B^{-1}=g(x)$, un polinomio en $X$ de grado $-n$. En este caso se obtiene

$$
A=B^{-1} g(x) B=g\left(B^{-1} X B\right)=g(L) .
$$

Definición 1.7 Sea $A \in \mathcal{L}$ con índice $m$, decimos que $A$ es mónica si todas las entradas de la diagonal de índice $m$ son iguales a 1 .

Se denota por $\mathcal{T}$ el conjunto de $(-1,1)$-matrices banda cuyas entradas en las diagonales de índice 1 y -1 no son todas cero. Las $(-1,1)$-matrices banda también pueden llamarse matrices tridiagonales. Nótese que el índice de $\mathcal{T}$ es -1 y que $\mathcal{T}$ es cerrado bajo transposición. 
EJEMPLo 1.5 Sea $C$ una matriz tridiagonal,

$$
C=\left(\begin{array}{llll}
0 & 1 & 0 & 0 \\
1 & 0 & 0 & 0 \\
0 & 1 & 0 & 0
\end{array}\right),
$$

entonces su matriz transpuesta sigue siendo una matriz tridiagonal, esto es,

$$
C^{t}=\left(\begin{array}{lll}
0 & 1 & 0 \\
1 & 0 & 1 \\
0 & 0 & 0 \\
0 & 0 & 0
\end{array}\right)
$$

Definición 1.8 Sean $A, B \in \mathcal{L}$. Se dice que $A$ y $B$ son diagonalmente similares si y sólo si existe una matriz diagonal invertible de índice cero $\mathrm{T}$, tal que $B=\mathrm{T}^{-1} A \mathrm{~T}$.

Proposición 1.9 (a) Si $L \in \mathcal{T}$ entonces $L$ es diagonalmente similar a un elemento mónico de $\mathcal{T}$.

(b) Si $L \in \mathcal{T}$ es mónico entonces hay una única matriz mónica $A \in \mathcal{G}$ tal que $A^{-1} L A=X$.

(c) Si $L, M \in \mathcal{T}$ entonces ellas son similares una a la otra.

(d) Si $L \in \mathcal{T}$ entonces $L$ es diagonalmente similar a $L^{t}$.

(e) Si $L \in \mathcal{T}$ es mónico y tiene entradas positivas en la diagonal de índice 1 entonces $L$ es diagonalmente similar a un elemento simétrico de $\mathcal{T}$.

\section{Demostración.}

(a) Definimos la sucesión $\rho$ como sigue: $\rho_{0}=1$ y $\rho_{k}=\ell_{0,1} \ell_{1,2} \ldots \ell_{k-1, k}$ para $k \geq 1$. Sea $\delta_{\rho}=$ $\operatorname{diag}\left(\rho_{0}, \rho_{1}, \rho_{2}, \ldots\right)$, la matriz diagonal de índice cero cuya entrada en $(k, k)$ es $\rho_{k}$. Un cálculo simple muestra que $\delta_{\rho} L \delta_{\rho}^{-1}$ es un elemento mónico de $\mathcal{T}$.

(b) Sea $L$ un elemento mónico de $\mathcal{T}$. Queremos encontrar un mónico $A=\left[a_{j, k}\right]$ en $\mathcal{G}$ tal que $L A=A X$. La igualdad de las entradas en la posición $(j+k, k)$ de $L A$ y $A X$ nos dan la ecuación

$$
\ell_{j+k, j+k-1} a_{j+k-1, k}+\ell_{j+k, j+k} a_{j+k, k}+a_{j+k+1, k}=a_{j+k, k-1} .
$$

Resolviendo para $a_{j+k+1, k}$ vemos que esta entrada en la $j+1$ diagonal puede ser calculada si tenemos $a_{j+k-1, k}$, la cual está en la $j-1$ diagonal, $a_{j+k, k}$ la cual está en la $j$ diagonal, y $a_{j+k, k-1}$, la cual está en la entrada anterior en la $j+1$ diagonal. Además podemos encontrar la entrada de $A$ por diagonales, empezando con la diagonal de índice 1, porque todas las entradas en la diagonal de índice cero son iguales a 1 . Podemos también encontrar las entradas en $A$ por filas.

(c) Se sigue inmediatamente de (b).

(d) Sea $\rho_{0}=1 \mathrm{y}$

$$
\rho_{k}=\frac{\ell_{0,1} \ell_{1,2} \cdots \ell_{k-1, k}}{\ell_{1,0} \ell_{2,1} \cdots \ell_{k, k-1}}, \quad k \geq 1 .
$$

Definimos $\Delta_{\rho}=\operatorname{diag}\left(\rho_{0}, \rho_{1}, \rho_{2}, \ldots\right)$. Un cálculo simple da

$$
\Delta_{\rho} L \Delta_{\rho}^{-1}=L^{t}
$$


(e) Sea $\gamma_{0}=1$ y $\gamma_{k}=\sqrt{\ell_{1,0} \ell_{2,1} \cdots \ell_{k, k-1}}$ para $k \geq 1$. Definimos $\delta_{\gamma}=\operatorname{diag}\left(\gamma_{0}, \gamma_{1}, \gamma_{2}, \ldots\right)$. Entonces es fácil ver que $\Delta_{\gamma}^{-1} L \Delta_{\gamma}$ es simétrica.

\subsection{Sucesiones de polinomios}

Sea $\left\{p_{k}\right\}_{k \geq 0}$ una sucesión de polinomios con coeficientes reales tal que $\operatorname{grad}\left(p_{k}(x)-x^{k}\right)<k$, y por esta razón, se dice que es una sucesión de polinomios mónicos. Se usa la base de potencias $\left\{x^{k}, k \geq 0\right\}$ para expresar los polinomios $p_{k}$ de la forma

$$
p_{k}(x)=\sum_{j=0}^{k} a_{k j} x^{j}, \quad a_{k k}=1, \quad k \geq 0 .
$$

Se usan los coeficientes $a_{k j}$ para formar la matriz infinita $A=\left(a_{k j}\right)$. Nótese que $A$ es un elemento mónico de $\mathcal{G}$. Así, se dice que $A$ es la matriz asociada con la sucesión $\left\{p_{k}\right\}_{k \geq 0}$, y tiene la forma

$$
A=\left(\begin{array}{cccccc}
1 & 0 & 0 & 0 & 0 & \ldots \\
a_{10} & 1 & 0 & 0 & 0 & \ldots \\
a_{20} & a_{21} & 1 & 0 & 0 & \ldots \\
a_{30} & a_{31} & a_{32} & 1 & 0 & \ldots \\
\vdots & \vdots & \vdots & \vdots & \vdots & \ddots
\end{array}\right)
$$

La $k$-ésima fila de $A$ corresponde a los coeficientes del polinomio $p_{k}(x)$. Es fácil ver que la $k$-ésima fila de $A X$ corresponde a los coeficientes de $x p_{k}(x)$, y la $k$-ésima fila de $A D$ corresponde a los coeficientes de $p_{k}^{\prime}(x)$, como se puede ver enseguida:

$$
A X=\left(\begin{array}{ccccc}
0 & 1 & 0 & 0 & \ldots \\
0 & a_{10} & 1 & 0 & \ldots \\
0 & a_{20} & a_{21} & 1 & \ldots \\
0 & a_{30} & a_{31} & a_{32} & \ldots \\
\vdots & \vdots & \vdots & \vdots & \ddots
\end{array}\right), \quad A D=\left(\begin{array}{ccccc}
0 & 0 & 0 & 0 & \ldots \\
1 & 0 & 0 & 0 & \ldots \\
a_{21} & 2 & 0 & 0 & \ldots \\
a_{31} & 2 a_{32} & 3 & 0 & \ldots \\
\vdots & \vdots & \vdots & \vdots & \ddots
\end{array}\right) .
$$

Definición 1.10 Sea $\mu$ un funcional lineal definido en el espacio de todos los polinomios. La sucesión de polinomios $\left\{p_{k}\right\}_{k \geq 0}$ es ortogonal con respecto a $\mu$ si y sólo si

$$
\mu\left(p_{k} p_{n}\right)=\gamma_{k} \delta_{k, n}, \quad k, n \in \mathbb{N},
$$

donde $\gamma_{k}$ son constantes reales distintas de cero. Si todos los $\gamma_{k}$ son positivos se dice que $\mu$ es un funcional lineal positivo.

Los momentos de $\mu$ son los números $\mu_{j}=\mu\left(x^{j}\right)$, para $j \geq 0$. Se define el vector columna de momentos por $\nu=\left(\mu_{0}, \mu_{1}, \mu_{2}, \ldots\right)^{t}$. Entonces

$$
\mu\left(p_{k}(x)\right)=\sum_{j=0}^{k} a_{k j} \mu_{j},
$$

es el $k$-ésimo elemento del vector columna $A \nu$ y

$$
\mu\left(x^{m} p_{k}(x)\right)=\sum_{j=0}^{k} a_{k j} \mu_{j+m},
$$


es el $k$-ésimo elemento del vector columna $A X^{m} \nu$.

Como cada $x^{n}$ es una combinación lineal de $p_{0}, p_{1}, \ldots, p_{n}$, la condición de ortogonalidad (1.1) es equivalente a

$$
\mu\left(x^{m} p_{k}(x)\right)=\left\{\begin{array}{l}
0, \quad \text { si } \quad 0 \leq m<k \\
\gamma_{k}, \quad \text { si } \quad m=k
\end{array}\right.
$$

Teorema 1.11 Sea $\left\{p_{k}\right\}_{k \geq 0}$ una sucesión de polinomios mónicos y sea $A$ la matriz asociada. Entonces, la sucesión $\left\{p_{k}\right\}_{k \geq 0}$ es ortogonal con respecto a algún funcional lineal si y sólo si la matriz $L=A X A^{-1}$ está en $\mathcal{T}$, es decir, $L=A X A^{-1}$ es una $(-1,1)$-matriz banda.

Demostración. Suponga que la sucesión $\left\{p_{k}\right\}_{k \geq 0}$ es ortogonal con respecto al funcional $\mu$, con vector de momentos $\nu$. Para $j \geq 0$ sea $e_{j}$ el vector columna infinito con la $j$-ésima entrada igual a 1 y todos los otros elementos iguales a cero. De la definición de $L$ se tiene $L^{m} A=A X^{m}$ para $m \geq 0$. Por lo tanto $L^{m} A \nu=A X^{m} \nu$, para $m \geq 0$. Por la condición de ortogonalidad (1.1) se ve que la $k$-ésima entrada de $A X^{m}$ es cero si $0 \leq m<k$ y ésta es $\gamma_{m} \neq 0$ si $m=k$. Por lo tanto, $A X^{m} \nu$ está en el subespacio generado por $\left\{e_{0}, e_{1}, \ldots, e_{m}\right\}$. La condición de ortogonalidad también da $A \nu=\gamma_{0} e_{0}$, y además $\gamma_{0} L^{m} e_{0}$ está en el subespacio generado por $\left\{e_{0}, e_{1}, \ldots, e_{m}\right\}$ y se puede escribir como una combinación lineal de éstos

$$
\gamma_{0} L^{m} e_{0}=\gamma_{m} e_{m}+\sum_{j=0}^{m-1} c_{m, j} e_{j}
$$

para algunos coeficientes $c_{m, j}$. Entonces

$$
\gamma_{0} L^{m+1} e_{0}=\gamma_{m} e_{m}+L \sum_{j=0}^{m-1} c_{m, j} e_{j}=\gamma_{m+1} e_{m+1}+\sum_{j=0}^{m} c_{m+1, j} e_{j}
$$

Por lo tanto

$$
L e_{m}=\frac{\gamma_{m+1}}{\gamma_{m}} e_{m+1}+\sum_{j=0}^{m} g_{m, j} e_{j},
$$

para algunos coeficientes $g_{m, j}$. Esto significa que todas las entradas de $L$ abajo de la diagonal de índice 1 son iguales a cero, y sus elementos en la diagonal de índice 1 para $m \geq 0$ son

$$
\ell_{m+1, m}=\frac{\gamma_{m+1}}{\gamma_{m}}
$$

Como $A$ y $A^{-1}$ son mónicas de índice cero y $X$ es mónica de índice -1 , es claro que $L$ es $(-1,1)$ matriz banda, mónica y pertenece a $\mathcal{T}$.

Ahora suponga que $\left\{p_{k}\right\}_{k \geq 0}$ es una sucesión de polinomios con matriz asociada $A$ en $\mathcal{G}$ tal que $L=A X A^{-1}$ es mónica de índice -1 . Además $L$ es $(-1,1)$-matriz banda, $L^{m}$ es $(-m, m)$-matriz banda y tiene entradas distintas de cero en su diagonal de índice $m$. De las ecuaciones $A X^{m} \nu=$ $L^{m} A \nu=L^{m} e_{0}$ se observa que la condición de ortogonalidad (1.2) se satisface y los polinomios $p_{k}$ son ortogonales con respecto al funcional $\mu$ cuyos momentos son las entradas en la 0-ésima columna de $A^{-1}$. Luego, de la ecuación $L^{m} e_{0}=A X^{m} \nu$, se tiene

$$
e_{0}^{t} L^{m} e_{0}=\left(e_{0}^{t} A\right) X^{m} \nu=e_{0}^{t} X^{m} \nu,
$$

y esto dice que la entrada $(0,0)$ de $L^{m}$ es igual a $\mu_{m}$. Por lo anterior, la sucesión de momentos se puede obtener de $L$, es decir, la entrada $(0,0)$ de las matrices $L^{m}$ corresponde al $m$-ésimo momento. 
La matriz $L$ del teorema anterior tiene la forma

$$
L=\left(\begin{array}{ccccccc}
\beta_{0} & 1 & 0 & 0 & 0 & 0 & \cdots \\
\alpha_{1} & \beta_{1} & 1 & 0 & 0 & 0 & \cdots \\
0 & \alpha_{2} & \beta_{2} & 1 & 0 & 0 & \cdots \\
0 & 0 & \alpha_{3} & \beta_{3} & 1 & 0 & \cdots \\
\vdots & \vdots & \vdots & \vdots & \vdots & \vdots & \ddots
\end{array}\right), \quad \text { donde } \alpha_{k}=\frac{\gamma_{k}}{\gamma_{k-1}}, \text { para } k \geq 1
$$

Igualando las $k$-ésimas filas de la ecuación matricial $L A=A X$, se obtiene la relación de recurrencia

$$
\alpha_{k} p_{k-1}(x)+\beta_{k} p_{k}(x)+p_{k+1}(x)=x p_{k}(x), \quad \text { para } \quad k \geq 1
$$

La relación de recurrencia (1.4) se utiliza para calcular los polinomios cuando conocemos $L$. Nótese que se tienen las condiciones iniciales $p_{0}(x)=1$ y $p_{1}(x)=x-\beta_{0}$.

Para $Z \in \mathcal{L}$ y $k \geq 1$ se define la $k$-sección de $Z$ como la matriz finita $k \times k Z_{(k)}=\left(z_{j, i}\right)$ donde $0 \leq j \leq k-1$ y $0 \leq i \leq k-1$. Tomando el producto de la $k$-sección de las matrices $A^{-1}, L$, y $A$ se obtiene

$$
A_{(k)}^{-1} L_{(k)} A_{(k)}=\left(\begin{array}{cccccc}
0 & 1 & 0 & 0 & \cdots & 0 \\
0 & 0 & 1 & 0 & \cdots & 0 \\
0 & 0 & 0 & 1 & \cdots & 0 \\
\vdots & \vdots & \vdots & \vdots & \ddots & \vdots \\
-a_{k, 0} & -a_{k, 1} & -a_{k, 2} & -a_{k, 3} & \cdots & -a_{k, k-1}
\end{array}\right) .
$$

Esta es la matriz compañera del polinomio mónico $p_{k}(x)=a_{k, 0}+a_{k, 1} x+a_{k, 2} x^{2}+\cdots+a_{k, k-1} x^{k-1}+x^{k}$. Por lo tanto, $p_{k}(x)$ es el polinomio característico de $L_{(k)}$. Es importante recordar que las matrices compañeras son no-derogatorias (una matriz cuyo polinomio característico y polinomio mínimo son iguales, es decir los valores propios tienen todos multiplicidad geométrica 1) y en consecuencia, las raíces de $p_{k}(x)$ son simples. Por otro lado, si $L$ tiene entradas positivas en la diagonal de índice 1 , es decir, $\alpha_{k}>0$ para $k \geq 1$, entonces $L$ es similar a una matriz simétrica y entonces $L_{(k)}$ es también similar a una matriz simétrica. Además, en tal caso todos los eigenvalores de $L_{(k)}$ son reales y entonces $p_{k}(x)$ tiene $k$ raíces reales distintas. En $(1.5)$ usamos $\left(A_{(k)}\right)^{-1}=\left(A_{(k)}^{-1}\right)$, que se cumple para $A \in \mathcal{G}$, pero en general, las operaciones de tomar la $k$-sección de matrices infinitas y la multiplicación, no conmutan.

Si $A$ es la matriz asociada a una sucesión de polinomios ortogonales mónicos, $\left\{p_{k}\right\}_{k \geq 0}$, se dirá que A es una matriz de polinomios ortogonales (mónicos), lo cual se abrevia con MPO, tal notación será aplicada para describir ciertas matrices y no usaremos la palabra abreviada para referirnos a sucesiones de polinomios.

\subsection{Sucesiones de polinomios ortogonales clásicos}

Sea $A$ una $M P O$ asociada con la sucesión de polinomios ortogonales mónicos $\left\{p_{k}\right\}_{k \geq 0}$. Entonces $A D$ tiene índice 1 y su $k$-ésima fila es el vector $\left(a_{k 1}, 2 a_{k 2}, 3 a_{k 3}, \ldots, k a_{k k}, 0, \ldots\right)$, la cual corresponde a la derivada de $p_{k}(x)$. Además la matriz $\hat{D} A D$ es un elemento mónico de $\mathcal{G}$ y su $k$-ésima fila corresponde al polinomio mónico $p_{k+1}^{\prime}(x) /(k+1)$. La $M P O A$ es llamada clásica si y sólo si la matriz $\hat{D} A D$ es también una $M P O$. Para cualquier matriz $Z \in \mathcal{L}$ se escribirá $\widetilde{Z}=\hat{D} Z D$. 
Teorema 1.12 Sea $A$ una $M P O$. Entonces $A$ es clásica si y sólo si $A \widetilde{A}^{-1}$ es una $(0,2)$-matriz banda y está en $\mathcal{G}$, donde $\widetilde{A}=\hat{D} A D$ es la matriz de derivadas con polinomios mónicos $\frac{p_{k+1}^{\prime}(x)}{k+1}$.

Demostración. Sea $A$ una $M P O$ y sea $L$ un elemento mónico de $\mathcal{T}$ que satisfacen $L A=A X$. Se definen $U=A \widetilde{A}^{-1}$ y $M=U^{-1} L U$. Está claro que $U \in \mathcal{G}$ y $M$ es una matriz mónica de índice -1 . Por lo definido anteriormente se tiene $M \widetilde{A}=U^{-1} L U \widetilde{A}=U^{-1} L A=U^{-1} A X=\widetilde{A} X$. Por el Lema 1.3 se obtiene

$$
A^{-1} D U^{-1} A=A^{-1} D \widetilde{A}=A^{-1} D \hat{D} A D=A^{-1} J A D=D,
$$

y por lo tanto $A D A^{-1}=D U^{-1}$. De la identidad básica $I=X D-D X$ se obtiene

$$
A=A X D-A D X=L A D-A D A^{-1} A X=L A D-A D A^{-1} L A=L A D-D U^{-1} L A .
$$

Multiplicando por la derecha por $A^{-1}$ y usando $A D A^{-1}=D U^{-1}$ se obtiene $I=L D U^{-1}-D U^{-1} L$, y multiplicando por $U$ a la derecha se obtiene

$$
U=L D-D U^{-1} L U=L D-D M .
$$

Como $L$ es un elemento mónico de $\mathcal{T}$ y $D$ es diagonal de índice 1 , la ecuación (1.6) muestra que $U$ es $(0,2)$-matriz banda si y sólo si $M$ es un elemento mónico de $\mathcal{T}$. Se concluye que $A$ es una $M P O$ clásica si y sólo si $U$ es un elemento mónico $(0,2)$-matriz banda de $\mathcal{G}$.

Sea $A$ una $M P O$ clásica y sean $L$ y $M$ elementos mónicos de $\mathcal{T}$ tal que $L A=A X$ y $M \widetilde{A}=\widetilde{A} X$. Por el Teorema 1.12 , la matriz $U=L D-D M$ es equivalente a $A \widetilde{A}^{-1}$ y satisface $L U=U M$. Esta última ecuación es igual a

$$
L^{2} D+D M^{2}=2 L D M,
$$

y esto permite encontrar las entradas de $M$ en términos de las de $L$ y también produce fórmulas explícitas para las entradas de $L$, descrita en (1.3), en términos de $\beta_{0}, \beta_{1}, \beta_{2}$ y $\alpha_{1}$. Tales fórmulas serán presentadas más adelante. Por la Proposición $1.9(d)$ existen matrices diagonales invertibles $\Delta_{\rho}$ y $\Delta_{\sigma}$ tales que $\Delta_{\rho} L \Delta_{\rho}^{-1}=L^{t}$ y $\Delta_{\sigma} M \Delta_{\sigma}^{-1}=M^{t}$. Entonces, de $U=L D-D M$ se tiene

$$
U^{t}=D^{t} L^{t}-M^{t} D^{t}=D^{t} \Delta_{\rho} L \Delta_{\rho}^{-1}-\Delta_{\sigma} M \Delta_{\sigma}^{-1} D^{t},
$$

y además

$$
\Delta_{\sigma}^{-1} U^{t} \Delta_{\rho}=\Delta_{\sigma}^{-1} D^{t} \Delta_{\rho} L-M \Delta_{\sigma}^{-1} D^{t} \Delta_{\rho}
$$

Sea $V=\Delta_{\sigma}^{-1} U^{t} \Delta_{\rho}$ y sea $E=\Delta_{\sigma}^{-1} D^{t} \Delta_{\rho}$. Entonces se obtiene $V=E L-M E$. Nótese que $V$ es una $(0,2)$-matriz banda con entradas distintas de cero en la diagonal de índice 0, y $E$ matriz diagonal de índice -1 . Tomando transpuesta en ambos lados de $L U=U M$ y usando la definición de $V$, se obtiene inmediatamente $V L=M V$. Ahora $L U V=U M V=U V L$ y además $U V$ conmuta con $L$. De la misma forma se obtiene que $V U M=V L U=M V U$ y entonces $V U$ conmuta con $M$.

Nótese que el $i n d(U V) \geq-2$ y entonces, por el Corolario 1.6, se tiene $U V=g(L)$ donde $g$ es un polinomio de grado a lo más 2. Es importante recalcar que $U$ está en $\mathcal{G}$ y por lo tanto es invertible. Entonces $M=U^{-1} L U$ y además

$$
V U=U^{-1}(U V) U=U^{-1} g(L) U=g\left(U^{-1} L U\right)=g(M) .
$$

De las ecuaciones $U=A \widetilde{A}^{-1}, M \widetilde{A}=\widetilde{A} X$ y $V U=g(M)$ se obtiene

$$
V A=V U \widetilde{A}=g(M) \widetilde{A}=\widetilde{A} g(X) .
$$


La igualdad de la $k$-ésima fila en la ecuación (1.7) da como resultado

$$
\frac{p_{k+1}^{\prime}(x)}{k+1} g(x)=V_{k, k} p_{k}(x)+V_{k, k+1} p_{k+1}(x)+V_{k, k+2} p_{k+2}(x) .
$$

La siguiente caracterización de polinomios ortogonales clásicos es esencialmente equivalente a la caracterización obtenida por Al Salam-Chihara [2] y por Maroni [21], usando diferentes enfoques.

Teorema 1.13 Sea $A$ una $M P O$. Entonces $A$ es clásica si y sólo si existe una $W(-2,0)$-matriz banda con entradas distintas de cero en la diagonal de índice cero, y un polinomio $q(X)$ de grado a lo más 2 tal que

$$
W A=\widetilde{A} q(X)
$$

Demostración. Se demostró en (1.7) que si $A$ es clásica entonces (1.8) se cumple con $W=V$ y $q=g$.

Ahora suponga que $A$ es una $M P O, A X A^{-1}=L \in \mathcal{T}$, y (1.8) se cumple. Sea $U=A \widetilde{A}^{-1}$. Es claro que $U \in \mathcal{G}$. Se demostrara que $U$ es $(0,2)$-matriz banda y $q(X)$ tiene grado a lo más 2 , se observa que todas las entradas no cero de $q(L)$ se encuentran entre las diagonales de índices -2 y 2 . Sea $k \geq 3$. Entonces la entrada $(k, 0)$ de la matriz $U W$, la cual es $u_{k, 0} w_{0,0}$, debe ser cero. Entonces $u_{k, 0}=0$, además, por hipótesis $w_{0,0} \neq 0$. Sea ahora $k \geq 4$. La $(k, 1)$ entrada de $U W$, la cual es $u_{k, 0} w_{0,1}+u_{k, 1} w_{1,1}$ debe ser cero. Por lo tanto, $u_{k, 1}$ debe ser cero. Procediendo de esta forma se observa que todas las entradas de $U$, abajo de la diagonal de índice 2 son cero. Además, $U$ es $(0,2)$-matriz banda, y $A$ es clásica, por el Teorema 1.12.

Sea $A$ una $M P O$ clásica. Utilizando la notación introducida anteriormente, recalcando que $L$ y $M$ son elementos mónicos de $\mathcal{T}$ tal que $L A=A X$ y $M \widetilde{A}=\widetilde{A} X$. También se tiene

$$
U=L D-D M=A \widetilde{A}^{-1}, V=E L-M E, L U=U M, V L=M V, \quad \text { y } \quad U V=g(L),
$$

donde $g$ es un polinomio de grado a lo más 2. La ecuación anterior permite escribir de dos formas el producto $U V$,

$$
U V=U E L-U M E=U E L-L U E \quad \text { y } \quad U V=L D V-D M V=L D V-D V L .
$$

Combinando estas ecuaciones se obtiene $(U E+D V) L=L(U E+D V)$ y por el Corolario 1.6 se debe tener $U E+D V=h(L)$, donde $h$ es un polinomio de grado 1, porque el índice de $U E+D V$ es igual a -1 . Un simple cálculo muestra que $U E+V D$ conmuta con $M$ y es un polinomio de grado 1 en $M$.

De $U=L D-D M$ y $V=E L-M E$ se obtiene $U E=L D E-D M E$ y $D V=D E L-D M E$. De lo anterior se tiene

$$
U E-D V=L D E-D E L .
$$

Se define la transformación $T_{A}: \mathcal{L} \rightarrow \mathcal{L}$ por $T_{A}(Z)=A^{-1} Z A$ para $Z \in \mathcal{L}$. Como $A$ es invertible y tiene índice cero, es claro que $T_{A}$ es un isomorfismo de álgebras que preserva índices. Nótese que $T_{A}(L)=X$. Calculamos a continuación $T_{A}(Z)$ para algunos valores particulares de $Z$. Como $U V=g(L)$, donde $g$ es un polinomio de grado a lo más 2, se obtiene $T_{A}(U V)=g\left(T_{A}(L)\right)=g(X)$. En la demostración del Teorema 1.12 se obtuvo $T_{A}\left(D U^{-1}\right)=D$. De $D V=D U^{-1} U V$ se tiene

$$
T_{A}(D V)=T_{A}\left(D U^{-1}\right) T_{A}(U V)=D g(X)
$$


Escribiendo $D E=D U^{-1} U E=D U^{-1}(h(L)-D V)$, se tiene

$$
T_{A}(D E)=T_{A}\left(D U^{-1}\right) T_{A}(h(L)-D V)=D(h(X)-D g(X)) .
$$

Por lo tanto, se ha obtenido la ecuación diferencial de Bochner

$$
\Lambda A=A\left(D h(X)-D^{2} g(X)\right)
$$

donde $\Lambda=D E$ es diagonal de índice cero. El polinomio $g$ en (1.9) es el mismo que aparece en (1.7). La ecuación (1.9) dice que la $k$-ésima fila de $A$ identificada con $p_{k}(x)$, satisface la ecuación diferencial

$$
\lambda_{k} p_{k}(x)=h(x) p_{k}^{\prime}(x)-g(x) p_{k}^{\prime \prime}(x), \quad k \geq 0,
$$

donde $\lambda_{k}$ es la entrada $(k, k)$ de la matriz diagonal $D E$. Nótese que $\lambda_{0}=0$ y $\lambda_{k} \neq 0$ para $k \geq 1$. Obsérvese también que $D h(X)-D^{2} g(X)$ es una (0,2)-matriz banda y su entrada en la posición $(0,0)$ es cero.

Teorema 1.14 Sea $A$ una $M P O$. Entonces $A$ es clásica si y sólo si existen polinomios $\psi$ de grado uno y $\phi$ de grado a lo más 2 , y una matriz diagonal $\Delta=\operatorname{diag}\left(\delta_{0}, \delta_{1}, \delta_{2}, \ldots\right)$ tal que $\delta_{0}=0$ y $\delta_{k} \neq 0$ para $k \geq 1$, que satisface

$$
\Delta A=A\left(D \psi(X)-D^{2} \phi(X)\right)
$$

Demostración. Anteriormente se demostró que si $A$ es clásica entonces (1.10) se satisface con $\Delta=\Lambda$, $\psi=h$ y $\phi=g$. Ahora suponga que (1.10) se cumple. Sea $U=A \widetilde{A}^{-1}$, como de costumbre, e introducimos la notación $B=D \psi(X)-D^{2} \phi(X)$. Por hipótesis $\Delta=D E$ con $E$ diagonal de índice -1. Nótese que $\widehat{D} \Delta=\widehat{D} D E$ entonces $\widehat{D} \Delta=J E=E$, además $E$ tiene inversa derecha. De la ecuación (1.10) $T_{A}(\Delta)=A^{-1} D E A=B$. Entonces

$$
T_{A}\left(D U^{-1} U E\right)=T_{A}\left(D U^{-1}\right) T_{A}(U E)=D T_{A}(U E)=B,
$$

y por lo anterior tenemos $T_{A}(U E)=\widehat{D} B=\psi(X)-D \phi(X)$. Sea $V=\widehat{D}(\psi(L)-U E)$. Entonces se tiene

$$
T_{A}(U E+D V)=\psi(X) \quad \text { y } \quad T_{A}(D V)=D \phi(X) .
$$

Por lo tanto $T_{A}(U E+D V)=\psi(X)$ y $T_{A}(D V)=D \phi(X)$. En consecuencia

$$
T_{A}(U E-D V)=\psi(X)-2 D \phi(X) .
$$

Utilizando la identidad $X D-D X=I$ es fácil ver que $X B-B X=\psi(X)-2 D \phi(X)$. Como $X B-B X=T_{A}(L \Delta-\Delta L)$ y $T_{A}$ es inyectiva, obtenemos $U E-D V=L \Delta-\Delta L$. Se tiene que $U E+D V=\psi(L)$ y además $U E-(\psi(L)-U E)=L \Delta-\Delta L$ entonces

$$
2 U E=\psi(L)+L \Delta-\Delta L
$$

También se tiene que $U E$ es una $(-1,1)$-matriz banda, y como $E$ es diagonal de índice -1 y tiene inversa diagonal de índice 1 , se obtiene que $U$ es $(0,2)$-matriz banda, y por el Teorema 1.12 se concluye que $A$ es clásica. 


\subsection{Construcción explícita de las matrices de polinomios ortogona- les clásicos}

Sea $A$ una $M P O$ clásica y sean $L$ y $M$ elementos mónicos de $\mathcal{T}$ que satisfacen $L A=A X$ y $M \widetilde{A}=\widetilde{A} X$, respectivamente. Escribiendo $L$ como en (1.3) y $M$ en una forma similar, con $\widetilde{\beta}_{j}$ en lugar de $\beta_{j}$ y $\widetilde{\alpha}_{j}$ en lugar de $\alpha_{j}$. Se probó en la sección anterior que $U=L D-D M=A \widetilde{A}^{-1} \mathrm{y}$ también que $L U=U M$. Es claro que las entradas de $U$ se pueden escribir en términos de las de $L$ y $M$, es decir, en términos de los $\widetilde{\beta}_{j}, \beta_{j}, \widetilde{\alpha}_{j}$ y $\alpha_{j}$. Nótese que $U$ es mónico y $(0,2)$-matriz banda, y como $L$ y $M$ son $(1,-1)$-matriz banda se puede ver que $L U$ y $U M$ son $(-1,3)$-matrices banda y mónicas. Además, la ecuación matricial $L U=U M$ es equivalente a cuatro ecuaciones vectoriales que corresponden a la igualdad de sus correspondientes diagonales de índices $0,1,2$ y 3 . Resolviendo estas ecuaciones se encuentran fórmulas explícitas para las sucesiones $\widetilde{\beta}_{j}, \beta_{j}, \widetilde{\alpha}_{j}$ y $\alpha_{j}$.

Para tal fin es conveniente definir las sucesiones $s_{k}$ y $d_{k}$ por

$$
s_{0}=\beta_{0} \quad \mathrm{y} \quad s_{k}=s_{k-1}+\beta_{k} \quad \text { para } \quad k \geq 1,
$$

y

$$
d_{0}=\beta_{0} \quad \mathrm{y} \quad d_{k}=\beta_{k}-\beta_{k-1} \quad \text { para } \quad k \geq 1
$$

Se observa que $s_{k}$ es la suma de las $\beta_{k}$, y $d_{k}$ es la diferencia de las $\beta_{k}$, y además

$$
\beta_{k}=s_{k}-s_{k-1} \quad \mathrm{y} \quad s_{k}=(k+1) d_{0}+k d_{1}+\cdots+2 d_{k-1}+d_{k} \quad \text { para } \quad k \geq 0 .
$$

Se introducen estas sucesiones porque las $s_{k}$ son funciones simples en términos de $\beta_{k}$, y escribiendo las fórmulas para las sucesiones relevantes en términos de $d_{0}, d_{1}, d_{2}$ se obtienen expresiones simples. Reemplazando las $\beta_{k}$ en la ecuación $L U=U M$, por $\beta_{k}=s_{k}-s_{k-1}$, y resolviendo las ecuaciones resultantes se encuentra que $s_{k}$ es una función racional de $k$ con numerador de grado 2 y denominador de grado 1, la cual está dada por

$$
s_{k}=(k+1)\left(d_{0}+\frac{k d_{1}\left(2 d_{1}+d_{2}\right)}{2\left(w(k)-3 d_{1}\right)}\right),
$$

donde $w(k)=\left(d_{1}-d_{2}\right) k+4 d_{1}+2 d_{2}$. También se obtiene

$$
\begin{gathered}
\alpha_{k}=\frac{k w(k-4)}{w(2 k-3) w(2 k-5)}\left(3\left(d_{1}+d_{2}\right) \alpha_{1}+(k-1) w(k-3)\left(\frac{d_{1}\left(2 d_{1}+d_{2}\right)}{w(2 k-4)}\right)^{2}\right), \\
\widetilde{\beta}_{k}=\frac{(k+1) s_{k+1}}{k+2}-\frac{k s_{k}}{k+1}, \quad \mathrm{y} \\
\widetilde{\alpha}_{k}=\frac{k w(k-2)}{(k+1) w(k-3)} \alpha_{k+1} .
\end{gathered}
$$

Nótese que $\alpha_{k}$ es una función racional de $k$, con numerador y denominador de grado 4 . Obsérvese que algunos denominadores en las fórmulas anteriores son cero si $d_{1}=0$ y $d_{2}=0$. Luego un cálculo directo con (1.12) muestra que tomando $d_{1}=0$ y $d_{2} \neq 0$ se obtiene $\alpha_{3}=-27 \alpha_{1}-d_{2}^{2}$ y $\alpha_{6}=0$; lo cual quiere decir que tales elecciones de $d_{1}, d_{2}$ no dan valores positivos para todos los $\alpha_{k}$, además algunos $\alpha_{k}$ son cero, lo cual se debe evitar, y si $d_{1}=0$ hay que tomar $d_{2}=0$. Es posible hacer frente a este caso de dos formas. Una es resolviendo la ecuación que resulta de $L U=U M$ haciendo 
$d_{1}=d_{2}=0$. Otra manera conveniente es eliminar $d_{2}$ de las fórmulas anteriores usando un nuevo parámetro $x$ de tal manera que las nuevas fórmulas funcionen cuando se toma $d_{1}=d_{2}=0$. Esto se hace de la siguiente manera. Sea

$$
d_{2}=\frac{4-5 x}{x-8} d_{1}
$$

esta función racional de $x$ se obtiene al tomar $x$ igual al coeficiente de $\alpha_{1}$ en la expresión para $\alpha_{2}$ dada por la fórmula (1.12). Definiendo

$$
u(k)=(2-x) k+x+4 .
$$

Usando (1.15) se elimina $d_{2}$ en las fórmulas (1.11), (1.12) y (1.14), obteniendo respectivamente

$$
\begin{gathered}
s_{k}=(k+1)\left(d_{0}-\frac{k d_{1}(x+4)}{2(3 x-2 u(k-2))}\right) \\
\alpha_{k}=\frac{k u(k-4)}{u(2 k-3) u(2 k-5)}\left(2(1+x) \alpha_{1}+(k-1) u(k-3)\left(\frac{d_{1}(x+4)}{2 u(2 k-4)}\right)^{2}\right), \quad \mathrm{y} \\
\tilde{\alpha}_{k}=\frac{k u(k-2)}{(k+1) u(k-3)} \alpha_{k+1} .
\end{gathered}
$$

Nótese que (1.13) no cambia introduciendo el parámetro $x$. Es fácil verificar que tomando $x$ en el intervalo $(0,2]$ entonces las sucesiones $s_{k}$ y $\alpha_{k}$ dadas por (1.17) y (1.18), respectivamente, están bien definidas y todas las $\alpha_{k}$ son positivas, siempre que $\alpha_{1}$ sea positiva.

La función racional de $x$ que aparece en (1.15) se obtiene tomando al coeficiente de $\alpha_{1}$ en la ecuación para $\alpha_{2}$ dada por la fórmula (1.12). Si $d_{1}=0$, entonces $\alpha_{2}=x \alpha_{1}$, y se observa que $x$ es un parámetro razonable cuando $d_{1}=d_{2}=0$.

Definimos el vector de parámetros $t=\left(d_{0}, d_{1}, x, \alpha_{1}\right)$ y denotamos el lado derecho de (1.17) por $F(t, k)$ y el lado derecho de (1.18) por $G(t, k)$. Esto es $F(t, k)=s_{k}$ y $G(t, k)=\alpha_{k}$. Definimos el conjunto

$$
\mathbf{T}=\left\{\left(y_{1}, y_{2}, y_{3}, y_{4}\right) \in \mathbb{R}^{4} \mid y_{3} \in(0,2], y_{4}>0\right\} .
$$

Con esta notación se puede resumir el resultado anterior como sigue, que también da una prueba directa del Teorema de Hahn.

Teorema 1.15 Cada MPO clásica corresponde a un par de sucesiones $\beta_{k}, \alpha_{k}$, dadas por

$$
\beta_{k}=F(t, k)-F(t, k-1), \quad k \geq 0 \quad y \quad \alpha_{k}=G(t, k), \quad k \geq 1
$$

para algún $t=\left(d_{0}, d_{1}, x, \alpha_{1}\right) \in \mathrm{T}$.

Definiendo la función $\delta: \mathrm{T} \rightarrow \mathrm{T}$ por

$$
\delta(t)=\left(d_{0}+\frac{d_{1}}{2}, \frac{3 d_{1} x}{8-x},(1+x)\left(\frac{\alpha_{1}}{3}+\frac{d_{1}^{2}}{12}\right)\right) .
$$

Nótese que $\delta$ está bien definida para $t \in \mathrm{T}$ y manda T sobre T. Un cálculo directo usando (1.13) y (1.19) produce

$$
\widetilde{\beta}_{k}=F(\delta(t), k)-F(\delta(t), k-1), \quad k \geq 0, \quad \text { y } \quad \widetilde{\alpha}_{k}=G(\delta(t), k), \quad k \geq 1 .
$$


Por lo tanto, la sucesión de derivadas de una sucesión de polinomios ortogonales clásica es también clásica. Esto es una prueba directa del Teorema de Hahn.

Nótese que una vez que se tienen las $s_{k}$ y las $\alpha_{k}$ se puede calcular fácilmente todas las matrices, vectores y polinomios asociados con $A$ y $\widetilde{A}$, tales como $M, U, V, E, \Lambda$, el vector de momentos $A^{-1} e_{0}$, y los polinomios $h$ y $g$ que aparecen en la ecuación diferencial de Bochner.

Consideramos ahora algunos casos particulares. Si $d_{1}=0$ entonces por (1.15) se debe tener $d_{2}=0$ y entonces $\beta_{k}=\beta_{0}=d_{0}$ para todo $k \geq 0$. Con estos valores la relación de recurrencia (1.4) muestra que en este caso todos los elementos de la sucesión de polinomios asociada son funciones de $z-\beta_{0}$. De (1.18) se observa que $\alpha_{k}$ en este caso es una función racional de $k$ con numerador y denominador de grado a lo más dos, y estos grados se pueden reducir tomando valores apropiados del parámetro $x$.

Una familia de casos relativamente simples se obtiene cuando $x=2$. De (1.16) se observa que tal elección produce casos particulares simples. Si $t=\left(d_{0}, d_{1}, 2, \alpha_{1}\right)$ entonces se obtiene

$$
s_{k}=(k+1) d_{0}+\left(\begin{array}{c}
k+1 \\
2
\end{array}\right) d_{1}
$$

y entonces

$$
\beta_{k}=d_{0}+k d_{1}, \quad k \geq 0
$$

También se tiene

$$
\alpha_{k}=k \alpha_{1}+\left(\begin{array}{c}
k \\
2
\end{array}\right) \frac{d_{1}^{2}}{2}, \quad k \geq 1 .
$$

Haciendo $d_{0}=\alpha_{1}$ y $d_{1}=2$ se obtienen los polinomios de Laguerre mónicos. Ver las tablas en el libro de Chihara [8]. Como $\delta\left(\alpha_{1}, 2,2, \alpha_{1}\right)=\left(1+\alpha_{1}, 2,2,1+\alpha_{1}\right)$ la sucesión de derivadas se obtiene reemplazando $\alpha_{1}$ con $1+\alpha_{1}$.

La intersección de las dos familias descritas anteriormente corresponde a vectores de parámetros de la forma $t=\left(d_{0}, 0,2, \alpha_{1}\right)$. Como $\delta\left(d_{0}, 0,2, \alpha_{1}\right)=\left(d_{0}, 0,2, \alpha_{1}\right)$ cada sucesión polinomial en esta familia es igual a su sucesión de derivadas. Los polinomios de Hermite mónicos pertenecen a esta familia. Ellos se obtienen con $t=(0,0,2,1 / 2)$ y sus coeficientes de recurrencia son $\beta_{k}=0$ para todo $k \geq 0$ y $\alpha_{k}=k / 2$ para $k \geq 1$.

Los polinomios de Jacobi mónicos con parámetros $s$ y $v-s$ forman otra importante familia. Dichos polinomios se obtienen cuando se toman

$$
d_{0}=\frac{v-2 s}{v+2}, \quad d_{1}=\frac{-4(v-2 s)}{(v+2)(v+4)}, \quad x=\frac{2(v+2)}{v+5}, \quad \alpha_{1}=\frac{4(1+s)(1+v-s)}{(v+2)^{2}(v+3)} .
$$

En este caso se tiene

$$
\begin{gathered}
\beta_{k}=\frac{v(v-2 s)}{(v+2 k)(v+2 k+2)}, \quad k \geq 0, \quad \mathrm{y} \\
\alpha_{k}=\frac{4 k(v+k)(s+k)(v-s+k)}{(v+2 k-1)(v+2 k)^{2}(v+2 k+1)}, \quad k \geq 1 .
\end{gathered}
$$

Tomando

$$
d_{0}=\frac{y}{v+2}, \quad d_{1}=\frac{-4 y}{(v+2)(v+4)}, \quad x=\frac{2(v+2)}{v+5}, \quad \alpha_{1}=\frac{-y^{2}}{(v+2)^{2}(v+3)},
$$


se obtiene una familia con parámetros y y $v$ que incluye los polinomios de Bessel.

Consideremos algunos ejemplos particulares. Los polinomios de Legendre mónicos se obtiene con los parámetros $t=(0,0,4 / 5,1 / 3)$. Como $\delta\left(d_{0}, 0,0, \alpha_{1}\right)=\left(d_{0}, 0,4 / 5, \alpha_{1} / 3\right)$, y $x=0$ no corresponden a una sucesión de polinomios con coeficientes de recurrencia positivos $\alpha_{k}$, se observa que los polinomios de Legendre no son la sucesión de derivadas de una sucesión clásica.

Los polinomios de Tchebyshev de primer tipo mónicos corresponden a $t=(0,0,1 / 2,1 / 2)$. Su sucesión de derivadas corresponde a los parámetros $(0,0,1,1 / 4)$ y estos son la sucesión de polinomios de Tchebyshev de segundo tipo mónicos. Estas sucesiones están incluidas en la familia de Jacobi. Por ejemplo, tomando los parámetros de Jacobi $s=-1 / 2$ y $v=-1$ tenemos los polinomios de Tchebyshev de primer tipo mónicos.

Los métodos matriciales que se han usado en este capítulo para estudiar los polinomios ortogonales clásicos también se pueden aplicar para estudiar otras sucesiones de polinomios clásicos, las cuales son básicamente sucesiones de polinomios que poseen la llamada propiedad de Hahn con respecto a un operador similar a la derivada. En otras palabras, estos últimos son esencialmente una colección de sucesiones de polinomios ortogonales que permanece ortogonal después de la acción de un operador lineal que decrece en una unidad el grado de un polinomio y produce 0 cuando actúa sobre una constante. Ejemplos de tales operadores son el operador diferencia y el operador $q$-derivada de Jackson. 


\section{CAPÍTULO 2}

\section{1 \\ LA CLASE DE HAHN EXTENDIDA DE SUCESIONES DE POLINOMIOS ORTOGONALES DISCRETOS}

En este Capítulo se amplían algunos de los resultados obtenidos en el capítulo anterior, donde se obtuvieron varias caracterizaciones y fórmulas explícitas para los coeficientes de recurrencia de todas las sucesiones de polinomios ortogonales clásicos. Aquí se obtienen fórmulas explícitas para los coeficientes de recurrencia de ciertas familias de sucesiones de polinomios ortogonales discretos. La estructura del Capítulo es la siguiente: en la sección 2.1 se construye una extension natural $\mathcal{H}_{w,+}$ de la clase de Hahn $\mathcal{H}_{w}$ de sucesiones ortogonales con respecto al operador en diferencias $\Delta_{w}$ usual en la retícula uniforme con incremento $w$. Véase [8]. Luego se encuentran los coeficientes de recurrencia y las ecuaciones en diferencias de valores propios para la clase extendida usando el método de matrices. En la Sección 2.2 se demuestra que la clase de Hahn $\mathcal{H}_{w,+}$ es un conjunto de matrices mónicas $A$ que satisfacen una ecuación $Y A(I+w D)=A D\left(h\left(X_{w}\right)+D f\left(X_{w}\right)\right)$ con $h$ de grado uno y $f$ de grado a lo más dos. Finalmente, resolviendo una ecuación matricial y comparando ciertas entradas se obtienen fórmulas para los coeficientes de $f$ en términos de $w$ y algunos coeficientes de la relación de recurrencia. Los coeficientes de $h$ se obtienen de la ecuación matricial $L A=A X_{w}$.

\subsection{Construcción explícita de matrices de polinomios ortogonales discretos}

Sea $w$ un número complejo distinto de cero. Se define el operador diferencia $\Delta_{w}$ sobre el espacio de polinomios con coeficientes complejos $\mathbb{C}[x]$ por

$$
\Delta_{w} p(x)=\frac{p(x+w)-p(x)}{w} .
$$

Denotamos por $\mathcal{N}_{w}$ la base de Newton de $\mathbb{C}[x]$ asociada con la sucesión $0, w, 2 w, 3 w, \ldots$, Los elementos de $\mathcal{N}_{w}$ son los polinomios $u_{k}$ definidos por $u_{0}(x)=1 \mathrm{y}$

$$
u_{k}(x)=x(x-w)(x-2 w) \cdots(x-(k-1) w), \quad k \geq 1 .
$$

Obsérvese que $\mathcal{N}_{w}$ es una base triangular para $\mathbb{C}[x]$. Un cálculo simple da

$$
\Delta_{w} u_{k}(x)=k u_{k-1}(x), \quad k \geq 0,
$$


$\mathrm{y}$

$$
x u_{k}(x)=u_{k+1}(x)+k w u_{k}(x), \text { para } k \geq 0 .
$$

Por lo tanto la representación matricial de $\Delta_{w}$ con respecto a la base $\mathcal{N}_{w}$ es la matriz $D$ definida en el capítulo anterior, y la representación del operador multiplicación $f(x) \rightarrow x f(x)$ con respecto a $\mathcal{N}_{w}$ es la matriz

$$
X_{w}=\left(\begin{array}{cccccc}
0 & 1 & 0 & 0 & 0 & \cdots \\
0 & w & 1 & 0 & 0 & \cdots \\
0 & 0 & 2 w & 1 & 0 & \cdots \\
0 & 0 & 0 & 3 w & 1 & \cdots \\
\vdots & \vdots & \vdots & \vdots & \vdots & \ddots
\end{array}\right) .
$$

Si $A \in \mathcal{G}$ es una matriz cuya $k$-ésima fila es considerada como el vector de coeficientes de un polinomio

$$
p_{k}(x)=\sum_{j=0}^{k} a_{k j} u_{j}(x), \quad a_{k k}=1, \quad k \geq 0,
$$

con respecto a la base $\mathcal{N}_{w}$ entonces la $k$-ésima fila de $A D$ es el vector de coeficientes de $\Delta_{w} p_{k}(x)$ y la $k$-ésima fila de $A X_{w}$ es el vector de coeficientes de $x p_{k}(x)$, ambos con respecto a $\mathcal{N}_{w}$. Es fácil ver que $D$ y $X_{w}$ satisfacen la identidad

$$
X_{w} D-D X_{w}=I+w D
$$

Entonces $\hat{D}$ es la inversa izquierda de $D$, es decir, $\hat{D} D=I$, y $D \hat{D}=J$. Si $A$ es un elemento mónico de $\mathcal{G}$ definimos $\widetilde{A}=\hat{D} A D$. Nótese que $\widetilde{A}$ es un elemento mónico de $\mathcal{G}$ y su $k$-ésima fila representa el polinomio normalizado (mónico)

$$
\frac{\Delta_{w} p_{k+1}}{k+1}, \quad \text { donde } \quad p_{k+1} \quad \text { corresponde a la } k+1 \text {-ésima fila de } A .
$$

Se define el conjunto $\mathcal{H}_{w}$, llamado la clase de Hahn como el conjunto de todas las MPO A tales que $\widetilde{A}$ es también una $M P O$. Se caracterizarán los elementos de $\mathcal{H}_{w}$ en términos de sus matrices de coeficientes de recurrencia.

TEOREMA 2.1

$$
L^{2} D-2 L D M+D M^{2}-w(L D-D M)=0
$$

Demostración. Suponiendo que $A \in \mathcal{H}_{w}$ y suponiendo además que $L$ es el único elemento de $\mathcal{T}$ tal que $L A=A X_{w}$. Como $A$ es una $M P O$ hay una única $M \in \mathcal{T}$ tal que $M \widetilde{A}=\widetilde{A} X_{w}$. Entonces se tiene

$$
M=\widetilde{A} A^{-1} L A \widetilde{A}^{-1}
$$

Sea $U=A \widetilde{A}^{-1}$. De la identidad (2.1) se obtiene

$$
\begin{aligned}
U & =A\left(X_{w} D-D X_{w}-w D\right) \widetilde{A}^{-1}=A X_{w} D \widetilde{A}^{-1}-A D X_{w} \widetilde{A}^{-1}-w A D \widetilde{A}^{-1} \\
& =L A D \widetilde{A}^{-1}-A D \widetilde{A}^{-1} M-w A D \widetilde{A}^{-1} \\
& =L D-D M-w D
\end{aligned}
$$

La igualdad anterior se sigue de

$$
A D \widetilde{A}^{-1}=A D \hat{D} \widetilde{A}^{-1} D=A J \widetilde{A}^{-1} D=D .
$$


Obsérvese que $A$ y $\widetilde{A}$ no aparecen en la expresión para $U$ obtenida anteriormente. Como $L U=U M$ y $U=L D-D M-w D$ se obtiene la ecuación (2.2).

Sean

$$
L=\left(\begin{array}{cccccc}
\beta_{0} & 1 & 0 & 0 & 0 & \ldots \\
\alpha_{1} & \beta_{1} & 1 & 0 & 0 & \ldots \\
0 & \alpha_{2} & \beta_{2} & 1 & 0 & \ldots \\
0 & 0 & \alpha_{3} & \beta_{3} & 1 & \ldots \\
\vdots & \vdots & \vdots & \vdots & \vdots & \ddots
\end{array}\right), \quad M=\left(\begin{array}{cccccc}
\widetilde{\beta}_{0} & 1 & 0 & 0 & 0 & \ldots \\
\widetilde{\alpha}_{1} & \widetilde{\beta}_{1} & 1 & 0 & 0 & \ldots \\
0 & \widetilde{\alpha}_{2} & \widetilde{\beta}_{2} & 1 & 0 & \ldots \\
0 & 0 & \widetilde{\alpha}_{3} & \widetilde{\beta}_{3} & 1 & \ldots \\
\vdots & \vdots & \vdots & \vdots & \vdots & \ddots
\end{array}\right)
$$

De la ecuación matricial (2.2) se obtienen todas las entradas de $L$ y $M$ en términos de $\beta_{0}, \beta_{1}, \beta_{2}, \alpha_{1}$ y $w$. Con el fin de obtener fórmulas algebraicas simples se utilizan las sucesiones $\gamma_{k}$ y $\delta_{k}$, es decir, la suma y diferencia de las $\beta_{k}$, respectivamente, definidas como sigue.

$$
\begin{aligned}
& \gamma_{k}=\beta_{0}+\beta_{1}+\ldots+\beta_{k}, \quad \text { para } k \geq 0, \\
& \delta_{k}=\beta_{k}-\beta_{k-1}, \quad \text { para } k \geq 1, \quad \text { con } \delta_{0}=\beta_{0} .
\end{aligned}
$$

Sea $\widetilde{\gamma}_{k}$ la suma de los $\widetilde{\beta}_{k}$. Los $\beta_{k}$ y $\widetilde{\beta}_{k}$ en (2.2) son reemplazados por sus correspondientes expresiones en términos de los $\gamma_{k}$ y los $\widetilde{\gamma}_{k}$. Obteniendo primero

$$
\begin{gathered}
\widetilde{\gamma}_{k}=(k+1)\left(\frac{\gamma_{k+1}}{k+2}-\frac{w}{2}\right), \quad \mathrm{y} \\
\widetilde{\alpha}_{k}=\frac{k g(k+1)}{(k+1) g(k)} \alpha_{k+1},
\end{gathered}
$$

donde $g(k)=c_{0} k+c_{1}, c_{0}=\delta_{1}-\delta_{2}$ y $c_{1}=\delta_{1}+5 \delta_{2}$. Resolviendo las ecuaciones escalares de (2.2) en un orden adecuado se obtiene

$$
\begin{gathered}
\gamma_{k}=(k+1)\left(\delta_{0}+\frac{\delta_{1} g(3) k}{2 g(2 k+1)}\right), \quad \mathrm{y} \\
\alpha_{k}=\frac{k g(k-1)}{g(2 k) g(2 k-2)}\left(g(2) \alpha_{1}-\frac{(k-1) g(k)}{4}\left(w^{2}-\left(\frac{\delta_{1} g(3)}{g(2 k-1)}\right)^{2}\right)\right) .
\end{gathered}
$$

Nótese que $\gamma_{k}$ depende solamente de $\delta_{0}, \delta_{1}, \delta_{2}$ y $k$, mientras que $\alpha_{k}$ depende solamente de $\delta_{1}, \delta_{2}, \alpha_{1}$, $w^{2}$, y $k$. Como $\delta_{k}=\beta_{k}-\beta_{k-1}$ vemos que la matriz $L$ está completamente determinada por cinco parámetros $\beta_{0}, \beta_{1}, \beta_{2}, \alpha_{1}$ y $w^{2}$, y las fórmulas (2.7) y (2.8). Por lo tanto, para un número complejo dado $w$ cada $M P O A \in \mathcal{H}_{w}$ está completamente determinada por los parámetros $\beta_{0}, \beta_{1}, \beta_{2}$ y $\alpha_{1}$. Nótese que $\mathcal{H}_{w}=\mathcal{H}_{-w}$.

De la ecuación (2.7) se observa que si $\delta_{1}=0$ entonces $\gamma_{k}=(k+1) \delta_{0}$ y por lo tanto $\delta_{2}$ debe ser igual a 0 . Como $g(k)$ no puede ser cero, si $\delta_{1} \neq 0$ entonces se debe tener

$$
\frac{\delta_{2}}{\delta_{1}} \neq \frac{k+1}{k-5}, \quad \text { para } \quad k \geq 0
$$

El caso limite $\delta_{2}=\delta_{1}$ es aceptable. Se puede obtener una extensión de la clase $\mathcal{H}_{w}$ usando en lugar de la ecuación (2.2), la ecuación matricial

$$
L^{2} D-2 L D M+D M^{2}-w(L D-D M)=t D,
$$

donde $t$ es un parámetro complejo. 
Definición 2.2 Se dice que una MPO A pertenece a $\mathcal{H}_{w,+}$ llamada la extensión de la clase de Hahn de $\Delta_{w}$, si y sólo si, la matriz $L$ de coeficientes de recurrencia de $A$ satisface la ecuación (2.9) para alguna matriz $M$ en $\mathcal{T}$ y algún número complejo $t$. La clase $\mathcal{H}_{w}$ corresponde a $t=0$.

Usando la Definición 2.2 y resolviendo (2.9) se obtienen nuevamente las ecuaciones (2.5), (2.6) y (2.7), pero en lugar de (2.8) ahora se obtiene

$$
\alpha_{k}=\frac{k g(k-1)}{g(2 k) g(2 k-2)}\left(g(2) \alpha_{1}-\frac{(k-1) g(k)}{4}\left(4 t+w^{2}-\left(\frac{\delta_{1} g(3)}{g(2 k-1)}\right)^{2}\right)\right) .
$$

Obsérvese que la única diferencia entre estas ecuaciones es que ahora tenemos $4 t+w^{2}$ en lugar de $w^{2}$.

Si $z$ y $w$ son números complejos y definimos $t=\left(z^{2}-w^{2}\right) / 4$, entonces se tiene $z^{2}=4 t+w^{2}$ y además si $A \in \mathcal{H}_{z}$ entonces estos coeficientes de recurrencia satisfacen la ecuación (2.7) y la ecuación (1.12) con $z$ en lugar de $w$, el cual coincide con la ecuación (2.10).

Por lo tanto, se tiene $\mathcal{H}_{z} \subset \mathcal{H}_{w,+}$. Por lo tanto, $\mathcal{H}_{w,+}=\mathcal{H}_{w_{1},+}$ para cualquier par de números complejos $w$ y $w_{1}$, por lo tanto, sólo hay una clase de Hahn extendida asociada con todos los operadores en diferencia $\Delta_{w}$, y es la unión de todos los conjuntos $\mathcal{H}_{w}$ para $w \in \mathbb{C}$.

\subsection{Ecuaciones en diferencias}

A continuación se mostrará que la clase de Hahn $\mathcal{H}_{w}$ es el conjunto de las matrices mónicas $A \in \mathcal{G}$ que satisfacen una ecuación matricial de la forma

$$
Y A(I+w D)=A B
$$

donde, $Y$ es diagonal de índice cero, la matriz $B$ es de la forma

$$
B=D\left(h\left(X_{w}\right)+D f\left(X_{w}\right)\right)
$$

con $h$ un polinomio de grado uno y $f$ es un polinomio de grado a lo más dos. Se escriben

$$
h\left(X_{w}\right)=h_{0} I+h_{1} X_{w} \quad \text { y } \quad f\left(X_{w}\right)=f_{0} I+f_{1} X_{w}+f_{2} X_{w}^{2} .
$$

Como $A$ y $I+w D$ son elementos mónicos de $\mathcal{G}$ es fácil ver que $Y$ debe ser igual a la diagonal de índice cero de $B$. Sea $Z=(I+w D)^{-1}$. Esta es una matriz invertible y triangular superior. Entonces (2.11) también se puede escribir como $Y A=A B Z$.

La derivada de Pincherle con respecto a $X_{w}$ de una matriz $P$ en $\mathcal{L}$ definida como

$$
P^{\prime}=X_{w} P-P X_{w}
$$

Nótese que $(P Q)^{\prime}=P^{\prime} Q+P Q^{\prime}$ y $\left(X_{w}^{k}\right)^{\prime}=0$ para $k \geq 0$. La identidad (2.1) es equivalente a $D^{\prime}=I+w D$, y de esta ecuación es fácil obtener $Z^{\prime}=-w Z$.

Usando las propiedades de la derivada de Pincherle un sencillo cálculo produce

$$
B^{\prime \prime}-3 w B^{\prime}+2 w^{2} B=2 f\left(X_{w}\right)-2 w h\left(X_{w}\right),
$$


y

$$
(B Z)^{\prime \prime}-w^{2} B Z=(I+w D)\left(2 f\left(X_{w}\right)-w h\left(X_{w}\right)\right) Z=2 f\left(X_{w}-w I\right)-w h\left(X_{w}-w I\right) .
$$

Las igualdades anteriores se siguen de $Z^{-1} X_{w} Z=X_{w}-w I$. La ecuación (2.13) se puede escribir como

$$
X_{w}^{2} B Z-2 X_{w} B Z X_{w}+B Z X_{w}^{2}-w^{2} B Z=2 f\left(X_{w}-w I\right)-w h\left(X_{w}-w I\right) .
$$

Suponga que $A$ es una matriz mónica en $\mathcal{G}$ que satisface (2.11) y sea $F=A X_{w} A^{-1}$. Obsérvese que $F$ se puede escribir como $F=L+T$ donde $L$ es como en (2.3) y $T$ es una semi-matriz inferior de índice 2. Como $A B Z A^{-1}=Y$, multiplicando ambos lados de (2.14) por $A$ del lado izquierdo y por $A^{-1}$ del lado derecho se deduce

$$
F^{2} Y-2 F Y F+Y F^{2}-w^{2} Y=2 f(F-w I)-w h(F-w I)
$$

Hay que recordar que las entradas distintas de cero de $Y$ son las entradas en la diagonal de índice cero de $B$ y, por lo tanto, pueden expresarse en términos de $w$ y los coeficientes de los polinomios $h$ y $f$. Resolviendo la ecuación obtenida y comparando las entradas $(0,1),(1,2)$ y $(0,0)$ en ambos lados de la ecuación (2.15) se obtienen los coeficientes $f_{1}, f_{2}, f_{0}$ en términos de $w, \gamma_{0}, \gamma_{1}, \gamma_{2}$ y $\alpha_{1}$. En consecuencia, de las correspondientes ecuaciones para las diagonales de índices -1 y 0 en (2.15) se obtienen las sucesiones $\gamma_{k}$ para $k \geq 3$, y $\alpha_{k}$, para $k \geq 2$ las cuales resultan ser las mismas sucesiones dadas por las fórmulas (2.7) y (2.8). Las ecuaciones de las diagonales de índice $1,2,3, \ldots$, producen $T=0$. Por lo tanto, $F=L \in T$ y $L$ es la matriz de recurrencia a tres términos de una $M P O$ en la clase $\mathcal{H}_{w}$. Pero se tiene $L A=A X_{w}$ y además $A$ está en $\mathcal{H}_{w}$.

Luego, el coeficiente $h_{0}$ se obtiene de la entrada $(0,0)$ de la ecuación matricial $L A=A X_{w}$ y $h_{1}$ permanece indeterminado y se puede considerar como un factor de escalamiento distinto de cero. Los coeficientes de $h$ y $f$ obtenidos en el procedimiento descrito anteriormente son

$$
\begin{aligned}
h_{0} & =h_{1}\left(w-\delta_{0}\right) \\
f_{0} & =\frac{h_{1}}{6 g(1)}\left(3 g(3) w^{2}-w g(5) \gamma_{2}+\delta_{0}\left(3 \delta_{1} g(3)+6 \delta_{0} c_{0}\right)-6 g(2) \alpha_{1}\right), \\
f_{1} & =\frac{h_{1}}{2 g(1)}\left(\delta_{1}(6 w-g(3))-4 \delta_{0} c_{0}\right) \\
f_{2} & =\frac{h_{1} c_{0}}{g(1)}
\end{aligned}
$$

y $h_{1}$ es cualquier número distinto de cero tal que $h_{1} \neq-2 f_{2}$, porque si $h_{1}+2 f_{2}=0$ entonces $g(3)=0$, y eso es no aceptable.

Las entradas en la matriz diagonal $Y$, llamadas los eigenvalores, están dadas por

$$
Y_{k, k}=h_{1} k\left(1+(k-1) \frac{c_{0}}{g(1)}\right), \quad k \geq 0 .
$$

Además, se puede observar que los eigenvalores dependen de $k, \delta_{1}, \delta_{2}$ y $h_{1}$, los cuales son esencialmente un factor de escalamiento. 
Si $w$ y los coeficientes $h_{0}, f_{0}, f_{1}, f_{2}$ son números dados entonces se pueden resolver las ecuaciones (2.16) para obtener los valores de los elementos $\delta_{0}, \delta_{1}, \delta_{2}$ y $\alpha_{1}$. Esto es, se obtiene

$$
\begin{aligned}
\delta_{0} & =\frac{h_{1} w-h_{0}}{h_{1}} \\
\delta_{1} & =\frac{4 h_{0} f_{2}+h_{1}^{2} w-2 h_{1} f_{1}}{h_{1}\left(2 f_{2}+h_{1}\right)} \\
\delta_{2} & =\frac{\left(4 h_{0} f_{2}+h_{1}^{2} w-2 h_{1} f_{1}\right)\left(h_{1}-2 f_{2}\right)}{h_{1}\left(2 f_{2}+h_{1}\right)\left(4 f_{2}+h_{1}\right)} \\
\alpha_{1} & =\frac{h_{0} h_{1} f_{1}-h_{0}^{2} f_{2}-f_{0} h_{1}^{2}}{h_{1}^{2}\left(f_{2}+h_{1}\right)}
\end{aligned}
$$

Nótese que el coeficiente $h_{1}$ se puede elegir tal que los denominadores en las ecuaciones (2.18) no se anulen. Entonces las fórmulas (2.7) y (2.8) dan las sucesiones $\gamma_{k}$ y $\alpha_{k}$, y por lo tanto la matriz de coeficientes de recurrencia $L$ está completamente determinada. La correspondiente MPO A está en $\mathcal{H}_{w}$ y satisface la ecuación matricial (2.11).

Se ha demostrado el siguiente teorema.

Teorema 2.3 La clase $\mathcal{H}_{w}$ es el conjunto de todas las MPOs A que satisfacen una ecuación en diferencias de la forma $Y A(I+w D)=A B$, donde $Y$ es matriz diagonal de índice cero, $y$

$$
B=D\left(h\left(X_{w}\right)+D f\left(X_{w}\right)\right),
$$

con $h$ es un polinomio de grado uno y $f$ es un polinomio de grado a lo más dos.

Ejemplos de elementos en la clase de Hahn, $\mathcal{H}_{w}$, son los siguientes.

Como bien se mencionó si $w$ y los coeficientes $h_{0}, f_{0}, f_{1}, f_{2}$ de los polinomios $h\left(X_{w}\right)$ y $f\left(X_{w}\right)$ son números dados se obtienen los valores de los elementos $\delta_{0}, \delta_{1}, \delta_{2}$ y $\alpha_{1}$, correspondientes a las entradas de la matriz $L$ cuyos elementos son los coeficientes de la relación de recurrencia de tres términos para la sucesión de polinomios.

Si el grado de $f\left(X_{w}\right)$ es cero entonces $f_{1}=f_{2}=0$ y podemos elegir $f_{0}=1$. Por otra parte, si $h_{1}<0$ y $h_{0}=h_{1}+1$ y $w=1$ entonces de las ecuaciones (2.18) se tiene

$$
\delta_{0}=\frac{h_{1}-h_{0}}{h_{1}}=\beta_{0}, \quad \delta_{1}=\delta_{2}=w=1, \quad \alpha_{1}=-\frac{1}{h_{1}} .
$$

Por tanto, de la ecuación (2.4) se obtienen

$$
\beta_{1}=\delta_{1}+\beta_{0}=\frac{2 h_{1}-h_{0}}{h_{1}}, \quad \beta_{2}=\delta_{2}+\beta_{1}=\frac{3 h_{1}-h_{0}}{h_{1}} .
$$

Estos coeficientes de la relación de recurrencia de tres términos coinciden con los obtenidos por las fórmulas dadas para los coeficientes de la relación de recurrencia para la familia de los polinomios de Charlier en el libro [14, pág. 97], tomando $w=1$.

Otra familia de polinomios ortogonales discretos se obtiene cuando el grado de $f\left(X_{w}\right)$ es uno, entonces $f_{2}=0$ y eligiendo $f_{1}=1, f_{0}=h_{0}-h_{1}+1, h_{0}>h_{1}$ y $w=1$ las ecuaciones (2.18) se convierten en 


$$
\delta_{0}=\frac{h_{1}-h_{0}}{h_{1}}=\beta_{0}, \quad \delta_{1}=\frac{h_{1}-2}{h_{1}}, \quad \delta_{2}=-\frac{1}{h_{1}}, \quad \alpha_{1}=-\frac{f_{0} h_{1}-h_{0}}{h_{1}^{2}} .
$$

Así, de la ecuación (2.4) se obtienen

$$
\beta_{1}=\delta_{1}+\beta_{0}=\frac{2 h_{1}-h_{0}-2}{h_{1}}, \quad \beta_{2}=\delta_{2}+\beta_{1}=\frac{2 h_{1}-h_{0}-3}{h_{1}} .
$$

Los coeficientes obtenidos coinciden con las fórmulas para la familia de los polinomios de Meixner en el libro [14, págs. 98 y 103], tomando $w=1$.

El estudio de los polinomios ortogonales discretos se encuentra en el Capítulo 5 del libro [14], las fórmulas explícitas obtenidas en éste para la relación de recurrencia y los coeficientes de la relación de recurrencia, los cuales son denotados por $c_{n}$ y $d_{n}$ aparecen en la ecuación (5.1.8) de la página 96.

El siguiente teorema es usualmente llamado el Teorema de Hahn.

Teorema 2.4 La clase $\mathcal{H}_{w}$ es cerrada bajo la transformación que manda $A$ en $\tilde{A}=\hat{D} A D$.

Demostración. Sea $A \in \mathcal{H}_{w}$. Entonces $A$ satisface la ecuación (2.11), que es,

$$
Y A(I+w D)=A D\left(h\left(X_{w}\right)+D f\left(X_{w}\right)\right)
$$

donde $Y, h$ y $f$ son como se definieron anteriormente, (2.16) y (2.17). Multiplicando ambos lados de la ecuación del lado izquierdo por $\hat{D}$ y del lado derecho por $D$, de este modo se obtiene

$$
\hat{D} Y D \hat{D} A D(I+w D)=\hat{D} A D\left(h\left(X_{w}\right)+D f\left(X_{w}\right)\right) D .
$$

Como $Y$ es diagonal y su entrada $(0,0)$ es cero, se tiene $Y=Y J=Y D \hat{D}$. Esto justifica la presencia del término $D \hat{D}$ justo después de la $Y$ en la ecuación anterior. Obsérvese que $D$ conmuta con $I+w D$ y también se observa que $\tilde{Y}=\hat{D} Y D$ es diagonal. Entonces se tiene

$$
\tilde{Y} \tilde{A}(I+w D)=\tilde{A}\left(h\left(X_{w}\right)+D f\left(X_{w}\right)\right) D .
$$

Usando la identidad $X_{w} D-D X_{w}=I+w D$ se puede mover a $D$ del lado izquierdo en la ecuación (2.19) obteniendo

$$
\left(h\left(X_{w}\right)+D f\left(X_{w}\right)\right) D=D h_{*}\left(X_{w}\right)+D^{2} f_{*}\left(X_{w}\right)+h_{1}(I+w D)
$$

donde

$$
h_{*}\left(X_{w}\right)=\left(h_{0}+f_{1}+w f_{2}\right) I+\left(h_{1}+2 f_{2}\right) X_{w},
$$

y

$$
f_{*}\left(X_{w}\right)=\left(f_{0}+w f_{1}+w^{2} f_{2}\right) I+\left(f_{1}+2 w f_{2}\right) X_{w}+f_{2} X_{w}^{2} .
$$

Sustituyendo en (2.19) se obtiene

$$
\tilde{Y} \tilde{A}(I+w D)=\tilde{A}\left(D h_{*}\left(X_{w}\right)+D^{2} f_{*}\left(X_{w}\right)\right)+h_{1} \tilde{A}(I+w D),
$$

y por tanto

$$
\left(\tilde{Y}-h_{1} I\right) \tilde{A}(I+w D)=\tilde{A}\left(D h_{*}\left(X_{w}\right)+D^{2} f_{*}\left(X_{w}\right)\right) .
$$


Esto significa que $\tilde{A}$ satisface una ecuación en diferencias de la forma (2.11) y entonces $\tilde{A}$ está en $\mathcal{H}_{w}$. Obsérvese que la entrada $(0,0)$ de $\tilde{Y}-h_{1} I$ es igual a cero y también que el coeficiente de $X_{w}$ en $h_{*}$ es igual $h_{1}+2 f_{2}$, el cual no es cero, y entonces $h_{*}$ tiene grado igual a uno.

Por último, si $A$ está en la clase extendida $\mathcal{H}_{w,+}$ entonces existe un número complejo $z$ tal que $A$ pertenece a la clase de Hahn $\mathcal{H}_{z}$ del operador en diferencias $\Delta_{z}$. Por lo tanto, con un adecuado cambio de base en $\mathbb{C}[x]$ que involucra las bases de Newton $\mathcal{N}_{z}$ y $\mathcal{N}_{w}$, se observará que la sucesión polinómica representada por $A$ con respecto a la base $\mathcal{N}_{w}$, está representada por una matriz $T$ con respecto a la base $\mathcal{N}_{z}$ y $T$ satisface una ecuación en diferencias de la forma (2.11), donde $z$ aparece en el lugar de $w$. 


\section{CAPÍTULO 3}

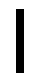 \\ LLA CLASE DE HAHN EXTENDIDA DE SUCESIONES DE POLINOMIOS $Q$-ORTOGONALES}

El objetivo de este Capítulo es encontrar fórmulas explícitas para los coeficientes de recurrencia de todas las sucesiones de polinomios $q$-ortogonales en la clase de Hahn extendida del operador en $q$-diferencias $\mathcal{D}_{q}$. El esquema del Capítulo es como sigue: en la Sección 3.1 se definen el operador $q$ derivada, su matriz de representación y la clase de Hahn, así como la clase de Hahn extendida cuyos elementos son matrices de polinomios ortogonales tal que la matriz tridiagonal $L$ de coeficientes de recurrencia satisface una ecuación matricial cuadrática, y se encuentran fórmulas explícitas para los coeficientes de la relación de recurrencia. Finalmente en la Sección 3.2 se caracteriza la clase de Hahn de acuerdo a una ecuación matricial de la forma $Y A E_{q}=A D_{q}\left(h(X)+D_{q} f(X)\right)$ donde $h$ es un polinomio de grado uno y $f$ un polinomio de grado a lo más dos. Se obtienen fórmulas para los coeficientes de los polinomios $h$ y $f$. Se muestra que es posible obtener los primeros coeficientes de la relación de recurrencia en términos de los coeficientes de esos dos polinomios.

\subsection{Coeficientes de la relación de recurrencia de sucesiones de po- linomios $q$-ortogonales}

Sea $q \in \mathbb{C} \neq 0$ que no es $k$-ésima raíz de la unidad. Se define el operador $q$-derivada sobre el espacio de polinomios $\mathbb{C}[x]$ de la siguiente forma

$$
\mathcal{D}_{q} p(x)=\frac{p(q x)-p(x)}{q x-x}, \quad p(x) \in \mathbb{C}[x],
$$

y los q-enteros como

$$
[k]=\frac{q^{k}-1}{q-1}, \quad k \in \mathbb{Z} .
$$

Si $k \geq 1$ se tiene $[k]=1+q+q^{2}+\ldots+q^{k-1}$. La acción de $\mathcal{D}_{q}$ sobre $p(x)=x^{k}$ es

$$
\mathcal{D}_{q} x^{k}=\frac{q^{k}-1}{q-1} x^{k-1}=[k] x^{k-1}, \quad k \geq 0 .
$$


Si $p(x)$ es diferenciable el operador $\mathcal{D}_{q}$ verifica

$$
\lim _{q \rightarrow 1} \mathcal{D}_{q} p(x)=\frac{d}{d x} p(x),
$$

el cual es el operador de derivación usual.

La matriz de representación para el operado $\mathcal{D}_{q}$ con respecto a la base de monomios $\left\{x^{n}\right\}_{n \geq 0}$, la cual se denotará por $D_{q}$, está dada por $\left(D_{q}\right)_{k+1, k}=[k+1]$ para $k \geq 0$ y todas las demás entradas son cero. Es decir, se tiene

$$
D_{q}=\left(\begin{array}{ccccc}
0 & 0 & 0 & 0 & \cdots \\
1 & 0 & 0 & 0 & \cdots \\
0 & 1+q & 0 & 0 & \cdots \\
0 & 0 & 1+q+q^{2} & 0 & \cdots \\
0 & 0 & 0 & 1+q+q^{2}+q^{3} & \cdots \\
\vdots & \vdots & \vdots & \vdots & \ddots
\end{array}\right)=\left(\begin{array}{ccccc}
0 & 0 & 0 & 0 & \cdots \\
{[1]} & 0 & 0 & 0 & \cdots \\
0 & {[2]} & 0 & 0 & \cdots \\
0 & 0 & {[3]} & 0 & \cdots \\
0 & 0 & 0 & {[4]} & \cdots \\
\vdots & \vdots & \vdots & \vdots & \ddots
\end{array}\right) .
$$

Como siempre, sea $X$ la representación matricial del operador de multiplicación que manda $p(x)$ a $x p(x)$ con respecto a la base $\left\{x^{k}, k \geq 0\right\}$. Las matrices $D_{q}$ y $X$ satisfacen la identidad

$$
X D_{q}-q D_{q} X=I
$$

La matriz $\hat{D}_{q}$ es la inversa izquierda de $D_{q}$, esto es, $\hat{D}_{q} D_{q}=I$ y $D_{q} \hat{D}_{q}=J$, y $\hat{D}_{q}$ tiene la forma

$$
\hat{D}_{q}=\left(\begin{array}{ccccc}
0 & 1 & 0 & 0 & \cdots \\
0 & 0 & \frac{1}{[2]} & 0 & \cdots \\
0 & 0 & 0 & \frac{1}{[3]} & \cdots \\
0 & 0 & 0 & 0 & \cdots \\
\vdots & \vdots & \vdots & \vdots & \ddots
\end{array}\right) .
$$

Definición 3.1 Se define la clase de Hahn $\mathcal{J}_{q}$ de $\mathcal{D}_{q}$ como el conjunto de todas las MPO A tal que

$$
\tilde{A}=\hat{D}_{q} A D_{q}
$$

es también una $M P O$.

Sea $A \in \mathcal{J}_{q}$ y sean $L, M \in \mathcal{T}$ tal que $L A=A X$ y $M \tilde{A}=\tilde{A} X$. Entonces

$$
M=\tilde{A} A^{-1} L A \tilde{A}^{-1}
$$

Sea $U=A \tilde{A}^{-1}$ usando la identidad (3.3) se tiene

$$
\begin{aligned}
U & =A\left(X D_{q}-q D_{q} X\right) \tilde{A}^{-1} \\
& =A X D_{q} \tilde{A}^{-1}-q A D_{q} X \tilde{A}^{-1} \\
& =L A D_{q} \tilde{A}^{-1}-q A D_{q} \tilde{A}^{-1} M \\
& =L D_{q}-q D_{q} M .
\end{aligned}
$$

Combinando las ecuaciones $L U=U M$ y $U=L D_{q}-q D_{q} M$ se obtiene el siguiente resultado. 
Teorema 3.2 Si $A$ está en $\mathcal{J}_{q}$ entonces

$$
L^{2} D_{q}-(q+1) L D_{q} M+q D_{q} M^{2}=0 .
$$

Definición 3.3 Se define la clase de Hahn extendida de $\mathcal{D}_{q}$, denotada por $\mathcal{J}_{q,+}$, como el conjunto de todas las MPOs A tales que su matriz $L \in \mathcal{T}$ de coeficientes de recurrencia satisface la ecuación matricial cuadrática

$$
L^{2} D_{q}-(q+1) L D_{q} M+q D_{q} M^{2}=t D_{q},
$$

para alguna $M \in \mathcal{T}$ y algún número complejo $t$.

Sean

$$
L=\left(\begin{array}{cccccc}
\beta_{0} & 1 & 0 & 0 & 0 & \ldots \\
\alpha_{1} & \beta_{1} & 1 & 0 & 0 & \ldots \\
0 & \alpha_{2} & \beta_{2} & 1 & 0 & \ldots \\
0 & 0 & \alpha_{3} & \beta_{3} & 1 & \ldots \\
\vdots & \vdots & \vdots & \vdots & \vdots & \ddots
\end{array}\right), \quad M=\left(\begin{array}{cccccc}
\widetilde{\beta}_{0} & 1 & 0 & 0 & 0 & \ldots \\
\widetilde{\alpha}_{1} & \widetilde{\beta}_{1} & 1 & 0 & 0 & \ldots \\
0 & \widetilde{\alpha}_{2} & \widetilde{\beta}_{2} & 1 & 0 & \ldots \\
0 & 0 & \widetilde{\alpha}_{3} & \widetilde{\beta}_{3} & 1 & \ldots \\
\vdots & \vdots & \vdots & \vdots & \vdots & \ddots
\end{array}\right)
$$

y sean $\sigma_{k}$ y $\tilde{\sigma}_{k}$ la suma de los $\beta_{k}$ y $\tilde{\beta}_{k}$, respectivamente. Se reemplazan los coeficientes $\beta_{k}$ y $\tilde{\beta}_{k}$ con sus correspondientes expresiones en términos de los $\sigma_{k}$ y $\tilde{\sigma}_{k}$ y entonces resolvemos la ecuación escalar obtenida por comparación de las entradas correspondientes en ambos lados de (3.4). Obteniendo primero

$$
\tilde{\sigma}_{k}=\frac{[k+1]}{[k+2]} \sigma_{k+1}, \quad k \geq 0,
$$

$\mathrm{y}$

$$
\tilde{\alpha}_{k}=\frac{[k] g(k+1)}{q[k+1] g(k)} \alpha_{k+1}, \quad k \geq 1,
$$

donde la función $g$ está definida por

$$
g(k)=[k-2] c_{0}+c_{1}, \quad k \geq 0,
$$

y las constantes $c_{0}$ y $c_{1}$ están definidas por

$$
c_{0}=[3]\left(q \sigma_{0}-\sigma_{1}\right)+\sigma_{2}, \quad c_{1}=[3]\left(\sigma_{0}+\sigma_{1}-\sigma_{2}\right) .
$$

Obsérvese que el parámetro $t$ no aparece en las ecuaciones (3.6) y (3.7). Del resto de las ecuaciones escalares obtenidas a partir de (3.4), se tiene

$$
\sigma_{k}=[k+1] \frac{c_{2}+[k+1] c_{3}}{g(2 k+1)}, \quad k \geq 0,
$$

$\mathrm{y}$, para $k \geq 1$

$$
\alpha_{k}=\frac{q[k] g(k-1)}{g(2 k) g(2 k-2)}\left(\frac{g(2) \alpha_{1}}{q^{2-k}}+[k-1] g(k)\left(\frac{\left(\left(q^{k-1}+1\right) \sigma_{0} c_{0}-c_{3}\right)\left(q^{k-2}\left(\sigma_{0} c_{0}-c_{3}\right)+\sigma_{0} c_{4}\right)}{q^{3-k}(g(2 k-1))^{2}}-t\right)\right),
$$

donde las constantes auxiliares $c_{2}, c_{3}, c_{4}$ están definidas por

$$
c_{2}=\frac{\sigma_{1} g(3)}{[2]}, \quad c_{3}=\frac{\sigma_{2} g(5)}{[3]}-\frac{\sigma_{1} g(3)}{[2]}, \quad c_{4}=g(3)-q g(2) .
$$

Nótese que la sucesión $\sigma_{k}$ es independiente del parámetro $t$. 


\subsection{Caracterización de la clase de Hahn $\mathcal{J}_{q}$}

Se caracterizará la clase de Hahn $\mathcal{J}_{q}$ como el conjunto de $M P O$ que satisfacen cierto tipo de ecuaciones. Sea $Y$ una matriz diagonal de índice cero y sea

$$
B=D_{q}\left(h(X)+D_{q} f(X)\right),
$$

donde $h$ es un polinomio de grado uno y $f$ es un polinomio de grado a lo más dos, esto es, se escriben

$$
h(X)=h_{0} I+h_{1} X \quad \text { y } \quad f(X)=f_{0} I+f_{1} X+f_{2} X^{2} .
$$

Sea $E_{q}$ la matriz de representación con respecto a la base canónica de monomios $\left\{x^{n}\right\}_{n \geq 0}$ de la función $p(x) \rightarrow p(q x)$. Obsérvese que $E_{q}$ es diagonal de índice cero y su entrada $(k, k)$ es igual a $q^{k}$, para $k \geq 0$. Es decir, tiene la forma

$$
E_{q}=\left(\begin{array}{cccccc}
1 & 0 & 0 & 0 & 0 & \ldots \\
0 & q & 0 & 0 & 0 & \ldots \\
0 & 0 & q^{2} & 0 & 0 & \ldots \\
0 & 0 & 0 & q^{3} & 0 & \ldots \\
\vdots & \vdots & \vdots & \vdots & \vdots & \ddots
\end{array}\right) .
$$

Sea $A$ un elemento mónico de $\mathcal{G}$ y considere la ecuación en $q$-diferencias

$$
Y A E_{q}=A B .
$$

Como $E_{q}$ es invertible es posible escribir (3.13) en la forma

$$
Y A=A B E_{q}^{-1} \text {. }
$$

Para simplificar la notación, sea $C=B E_{q}^{-1}$. Como $A$ es un elemento mónico de $\mathcal{G}$ se observa que $Y$ coincide con la diagonal de índice cero de $C$. Nótese que $C$ es una $(0,2)$-matriz banda.

Definición 3.4 Sean $F \in \mathcal{L}$ y $v \in \mathbb{C}$. Se define el $(F, v)$-operador de diferenciación de Pincherle como

$$
P_{F, v}(Z)=F Z-v Z F
$$

para $Z \in \mathcal{L}$.

La identidad (3.3) es equivalente entonces a $P_{X, q}\left(D_{q}\right)=I$.

Nótese que $P_{X, q}(X)=(1-q) X^{2}$. Además que $P_{X, 1}(Z)=0$ si y sólo si $Z$ es una matriz de Toeplitz, por la Proposición 1.5.

Sea $G=P_{X, q}\left(P_{X, \frac{1}{q}}(C)\right)$, realizando un cálculo sencillo se obtiene el resultado $P_{X, 1}(G)=0$. Como $G$ es una $(-2,0)$-matriz banda, ésta debe ser un polinomio en $X$ de la forma

$$
G=d_{0} I+d_{1} X+d_{2} X^{2} .
$$

Como $G$ es de Toeplitz, los coeficientes en (3.14) están dados por

$$
\begin{aligned}
& d_{0}=G_{0,0}=[2] f_{0}, \\
& d_{1}=G_{0,1}=\frac{[2]}{q} f_{1}+(1-q) h_{0}, \\
& d_{2}=G_{0,2}=\frac{[2]}{q^{2}} f_{2}+\frac{(1-q)}{q} h_{1} .
\end{aligned}
$$


Lema 3.5 (i) Sea $M=L+T$, donde $L$ es como en (3.5) y $T$ es una semi-matriz inferior de índice dos. Sea $Y=\operatorname{diag}\left(y_{0}, y_{1}, y_{2}, y_{3}, \ldots,\right)$ una matriz diagonal de índice cero con $y_{0}=0, y_{1} \neq 0$ y tal que $P_{M, q}\left(P_{M, \frac{1}{q}}(Y)\right)$ conmuta con $M$. Entonces $T=0$,

$$
y_{k}=\frac{y_{1}[k] g(k)}{g(1) q^{k-1}}, \quad k \geq 2,
$$

donde $g(k)=[k-2] c_{0}+c_{1}, k \geq 0, P_{L, q}\left(P_{L, \frac{1}{q}}(Y)\right)$ es un polinomio en $L$ de grado a lo más dos, y $L$ es la matriz de coeficientes de recurrencia de una MPO A en la clase de Hahn $\mathcal{J}_{q}$ de $\mathcal{D}_{q}$.

(ii) Sea $A \in \mathcal{J}_{q}$ y sea $L \in \mathcal{L}$ tal que $L A=A X$. Sea $y_{1} \neq 0$ y definimos $Y=\operatorname{diag}\left(0, y_{1}, y_{2}, y_{3}, \ldots,\right)$ donde $y_{k}$ está dado por (3.15) para $k \geq 2$. Entonces $P_{L, q}\left(P_{L, \frac{1}{q}}(Y)\right)$ es un polinomio en $L$ de grado a lo más dos.

\section{Demostración.}

(i) Sea $H=P_{M, q}\left(P_{M, \frac{1}{q}}(Y)\right)$. Por hipótesis se tiene que las matrices $M$ y $H$ conmutan, es decir, $M H=H M$. Recuérdese que $\beta_{k}=\sigma_{k}-\sigma_{k-1}$. Comparando las diagonales de índice -3 en la ecuación matricial $M H=H M$ se encuentra $y_{k}$ para $k \geq 3$, en términos de $q, y_{1}$ y $y_{2}$. Luego, a partir de la ecuación correspondiente a la entrada $(0,2)$ se obtiene $y_{2}$ en términos de $q, \sigma_{0}$, $\sigma_{1}, \sigma_{2}$ y $y_{1}$. La sustitución de la expresión obtenida para $y_{2}$ en la fórmula para las $y_{k}$, para $k \geq 3$, produce la fórmula (3.15).

De la diagonal de índice -2 se encuentra $\sigma_{k}$ para $k \geq 3$, en términos de $q, \sigma_{0}, \sigma_{1}, \sigma_{2}$, y de la diagonal de índice -1 se encuentra $\alpha_{k}$ para $k \geq 2$ en términos de $q, \sigma_{0}, \sigma_{1}, \sigma_{2}$ y $\alpha_{1}$. Luego, resolviendo en forma recursiva las ecuaciones obtenidas de las diagonales de índices $0,1,2, \ldots$, en $M H=H M$ se observa que las diagonales de índices $2,3,4, \ldots$ de $T$ son todas iguales a cero y entonces $T=0$ y $M=L$. Así $P_{L, q}\left(P_{L, \frac{1}{q}}(Y)\right)$ conmuta con $L$.

Las expresiones obtenidas para las sucesiones $\sigma_{k}$ y $\alpha_{k}$ coinciden con las obtenidas en (3.10) y (3.11), con $t=0$, y además $L$ es la matriz de coeficientes de recurrencia de una MPOA que pertenece a $\mathcal{J}_{q}$ y $L A=A X$.

Como $L$ es similar a $X$ y $P_{L, q}\left(P_{L, \frac{1}{q}}(Y)\right)$ conmuta con $L$ es fácil ver que $P_{L, q}\left(P_{L, \frac{1}{q}}(Y)\right)$ debe ser un polinomio en $L$ de grado a lo más dos. Ver Corolario 1.6.

(ii) Observando que las entradas de $L$ satisfacen las ecuaciones (3.10) y (3.11), con $t=0$. Un cálculo sencillo muestra que $P_{L, q}\left(P_{L, \frac{1}{q}}(Y)\right)$ conmuta con $L$ y tiene índice -2 . Como $L$ es similar a $X$ se concluye que $P_{L, q}\left(P_{L, \frac{1}{q}}(Y)\right)$ es un polinomio en $L$ de grado a lo más 2 . 
Teorema 3.6 Sea $A$ un elemento mónico de $\mathcal{G}$. Entonces $A$ está en la clase $\mathcal{J}_{q}$ si y sólo si $A$ satisface la ecuación matricial $Y A E_{q}=A B$ para alguna $B=D_{q}\left(h(X)+D_{q} f(X)\right)$ donde $h$ es un polinomio de grado uno y $f$ un polinomio de grado a lo más dos.

Demostración. Sea $A \in \mathcal{G}$ mónica que satisface $Y A E_{q}=A B$ para algún $B$ como se definió anteriormente, y sea $C=B E_{q}^{-1}$. Entonces, por (3.14) se tiene

$$
G=d_{0} I+d_{1} X+d_{2} X^{2}=P_{X, q}\left(P_{X, \frac{1}{q}}(C)\right) .
$$

Sea $M=A X A^{-1}$. Nótese que $M=L+T$ con $L$ y $T$ como en el Lema 3.5. Como $A C A^{-1}=Y$ y $A X A^{-1}=M$, de (3.16) se obtiene

$$
A G A^{-1}=A P_{X, q}\left(P_{X, \frac{1}{q}}(C)\right) A^{-1}=d_{0} I+d_{1} M+d_{2} M^{2}
$$

Considérese que $G=X^{2} C-\left(\frac{q+1}{q}\right) X C X+C X^{2}$. Entonces es claro que

$$
A P_{X, q}\left(P_{X, \frac{1}{q}}(C)\right) A^{-1}=P_{M, q}\left(P_{M, \frac{1}{q}}(Y)\right) .
$$

De la ecuación (3.17) se observa que $P_{M, q}\left(P_{M, \frac{1}{q}}(Y)\right)$ conmuta con $M$. Como $Y$ coincide con la diagonal de índice cero de $C$, es fácil ver que las hipótesis del Lema $3.5(i)$ se satisfacen y además se debe tener que $A \in \mathcal{J}_{q}$.

Ahora suponga que $A \in \mathcal{J}_{q}$ y sea $L \in \mathcal{T}$ tal que $L A=A X$. Sea $y_{1} \neq 0$ y sea $Y$ la matriz diagonal dada por el Lema 3.5 (ii). Definimos $C=A^{-1} Y A$. Note que $C$ tiene índice cero y su diagonal de índice cero coincide con la de $Y$. Como $P_{L, q}\left(P_{L, \frac{1}{q}}(Y)\right)=d_{0} I+d_{1} L+d_{2} L^{2}$ para algunas constantes $d_{0}, d_{1}, d_{2}$ que son fáciles de calcular, multiplicando por $A^{-1}$ del lado izquierdo y multiplicando por $A$ del lado derecho ambos lados de la ecuación anterior se obtiene

$$
P_{X, q}\left(P_{X, \frac{1}{q}}(Y)\right)=d_{0} I+d_{1} X+d_{2} X^{2}
$$

Este es un caso particular de la derivada de Pincherle

$$
P_{X, q}\left(P_{X, \frac{1}{q}}(R)\right)=e_{0} I+e_{1} X+e_{2} X^{2}
$$

donde las constantes $e_{0}, e_{1}, e_{2}$ están dadas y la matriz desconocida $R$ es de índice cero. Resolviendo la ecuación (3.18) por diagonales, iniciando con la diagonal de índice -2 , se pueden encontrar todas las entradas de $R$ en términos de $q, e_{0}, e_{1}, e_{2}, R_{0,0}, R_{1,0}$ y $R_{1,1}$. La solución general $R$ de (3.18) es una $(0,2)$-matriz banda. Si $R_{0,0}=0$ entonces $R$ se puede expresar como

$$
D_{q}\left(h(X)+D_{q} f(X)\right) E_{q}^{-1}
$$

donde $h$ es un polinomio de grado uno y $f$ es un polinomio de grado a lo más dos. Se puede expresar de otras maneras, por ejemplo, como un operador diferencial en términos de las matrices $D_{q}$ y $D_{\frac{1}{q}}$. 
Puesto que la diagonal de índice cero de $C$ coincide con la de $Y$, se tiene $C_{0,0}=0$ y $C_{1,1}=y_{1}$. Comparando las entradas $(1,0)$ en $Y A=A C$ se obtiene $C_{1,0}=-y_{1} \sigma_{0}$. Luego, usando los valores conocidos de los parámetros $d_{0}, d_{1}, d_{2}, C_{0,0}, C_{1,0}$ y $C_{1,1}$ se obtiene

$$
C=D_{q}\left(h(X)+D_{q} f(X)\right) E_{q}^{-1},
$$

donde $h(X)=h_{0} I+h_{1} X, f(X)=f_{0} I+f_{1} X+f_{2} X^{2}, \mathrm{y}$

$$
\begin{aligned}
h_{0} & =-y_{1} \sigma_{0}, \quad h_{1}=y_{1} q, \\
f_{0} & =\frac{y_{1}}{[2] q g(1)}\left(\sigma_{0} \sigma_{1} g(3)-[2] g(2)\left(\alpha_{1}+\sigma_{0}^{2}\right)\right), \\
f_{1} & =-\frac{y_{1} c_{3}}{q g(1)}, \quad f_{2}=\frac{y_{1} c_{0}}{g(1)} .
\end{aligned}
$$

Las constantes $c_{0}$ y $c_{3}$ fueron definidas por (3.9) y (3.12), respectivamente. Además $A$ satisface $Y A=A C$ lo cual es equivalente a $Y A E_{q}=A B$, y esto completa la demostración del teorema.

Es posible obtener $\sigma_{0}, \sigma_{1}, \sigma_{2}, \alpha_{1}$ en términos de $h_{0}, f_{0}, f_{1}, f_{2}$ resolviendo las ecuaciones (3.20). Obteniendo

$$
\begin{aligned}
\sigma_{0} & =-\frac{h_{0}}{y_{1}} \\
\sigma_{1} & =-\frac{[2] q\left(h_{0}+f_{1}\right)}{[2] f_{2}+q y_{1}} \\
\sigma_{2} & =-\frac{[3] q\left(h_{0}+[2] f_{1}\right)}{[4] f_{2}+q y_{1}} \\
\alpha_{1} & =-\frac{f_{2} h_{0}^{2}+q^{2} f_{0} y_{1}^{2}-q y_{1} h_{0} f_{1}}{y_{1}^{2}\left(q y_{1}+f_{2}\right)} .
\end{aligned}
$$

Hacemos notar que la elección $y_{1}=g(1)$ simplifica los coeficientes en la expresión para la matriz $C$ y también simplifica la fórmula (3.15) correspondiente a los eigenvalores $y_{k}$.

Consideremos ahora las $M P O$ en la clase extendida $\mathcal{J}_{q,+}$. Sea $A_{t}$ una MPO con coeficientes de recurrencia $L_{t}$ cuyas entradas están dadas por (3.10) y (3.11) para un $t$ distinto de cero. Sea $L_{0}$ la matriz de coeficientes de recurrencia obtenida de $L_{t}$ haciendo $t=0$. Obsérvese que $L_{t}$ y $L_{0}$ difieren sólo en la diagonal de índice 1 . Sea $A_{0}$ una $M P O$ que corresponde a $L_{0}$, es decir, el único elemento mónico de $\mathcal{G}$ que satisface $L_{0} A_{0}=A_{0} X$. Entonces $A_{0}$ está en $\mathcal{J}_{q}$ y por el Lema 3.5 existe $C$ dada por (3.19) tal que $Y A_{0}=A_{0} C$, donde $Y$ es la matriz diagonal de índice cero que coincide con la diagonal de índice cero de $C$.

Definiendo

$$
C_{t}=A_{t}^{-1} Y A_{t} \quad \text { y } \quad U=A_{t}^{-1} A_{0} .
$$

Entonces se tiene $Y A_{t}=A_{t} C_{t}$ y $C_{t}=U C U^{-1}$. Nótese que $U$ está en $\mathcal{G}$ y que el mapeo que manda $Z \in \mathcal{L}$ a $Z U$ se puede ver como un cambio de base. Obsérvese que la diagonal de índice 1 de $U$ es igual a cero. Dado que las ecuaciones anteriores arrojan $Y A_{t} U=A_{t} U C$, se ha demostrado el siguiente teorema.

Teorema 3.7 Sea $R$ un elemento de la clase extendida $\mathcal{J}_{q,+}$. Entonces existe una matriz mónica $U$ en $\mathcal{G}$ tal que $R U$ está en $\mathcal{J}_{q}$ y por lo tanto $R U$ satisface una ecuación del tipo (3.13). 


\section{CAPÍTULO 4 LPOLINOMIOS ORTOGONALES Q-CLÁSICOS QUE TIENEN SUCESIONES SIMPLES DE MOMENTOS}

La importancia de las sucesiones de momentos asociadas con polinomios ortogonales es bien conocida, puesto que todas las propiedades relevantes de los polinomios ortogonales pueden obtenerse a partir de los momentos. También es bien sabido que para ser la sucesión de momentos de polinomios ortogonales, una sucesión de números debe satisfacer ciertas condiciones expresadas en términos de determinantes de Hankel. Si consideramos clases restringidas de polinomios ortogonales, por ejemplo los polinomios ortogonales que satisfacen alguna ecuación diferencial o en diferencias, entonces las correspondientes sucesiones de momentos también están restringidas de alguna manera. En el caso de los polinomios ortogonales $q$-clásicos, que satisfacen una ecuación en $q$-diferencias de segundo orden, la sucesión de momentos está completamente determinada por sus cinco términos iniciales. En este Capítulo se estudian las sucesiones de momentos de polinomios ortogonales $q$-clásicos obteniendo una relación de recurrencia de tres términos con coeficientes variables que dependen de los coeficientes de la ecuación en $q$-diferencias satisfecha por los polinomios, presentado en [4]. Considerando los casos en los cuales la relación de recurrencia se simplifica se obtienen algunas familias de polinomios ortogonales $q$-clásicos que tienen fórmulas explícitas simples para los momentos, expresadas en términos de los parámetros en la ecuación en $q$-diferencias. Para cada familia, se encuentran fórmulas explícitas para los coeficientes de la relación de recurrencia de tres términos que satisfacen los polinomios ortogonales. Tales fórmulas permiten identificar las sucesiones de polinomios ortogonales $q$-clásicas que corresponden a nuestros ejemplos usando las relaciones de recurrencia normalizadas listadas en el Capítulo 14 del libro [14]. Resulta que nuestros ejemplos están relacionados con polinomios $q$-Krawtchouk y algunos polinomios $q$-Krawtchouk no estándar para los cuales el parámetro $N$ es un índice negativo, polinomios $q$-Bessel, polinomios $q$-Laguerre/Wall pequeños y polinomios $q$-Jacobi grandes.

La estructura del Capítulo es la siguiente: en la Sección 4.1 se obtiene la relación de recurrencia de tres términos satisfecha por los momentos, la cual se obtiene usando matrices infinitas. Los coeficientes en dicha relación de recurrencia son determinados por los coeficientes polinomiales de la ecuación en $q$-diferencias de segundo orden satisfecha por los polinomios ortogonales. En la Sección 4.2 se obtienen algunas familias de sucesiones de polinomios ortogonales q-clásicos que tienen sucesiones simples de momentos. Ésto se logra escogiendo de manera apropiada los parámetros en la ecuación en $q$-diferencias. Se obtienen dos grandes familias de sucesiones de polinomiales $q$-ortogonales para 
las cuales la recurrencia de los momentos se convierte en una recurrencia a dos términos, lo que produce fórmulas explícitas simples para los momentos. En la Sección 4.3 se presentarán las familias de sucesiones $q$-ortogonales que tienen momentos muy simples y ejemplos particulares.

\subsection{Sucesiones de polinomios ortogonales $q$-clásicos}

Se obtendrá una relación de recurrencia de tres términos para los momentos de sucesiones de polinomios ortogonales $q$-clásicos.

Recuérdese que las matrices $D_{q}$ y $X$ satisfacen la identidad $X D_{q}-q D_{q} X=I$. Sean $h(t)=h_{0}+h_{1} t$ y $g(t)=g_{0}+g_{1} t+g_{2} t^{2}$, y considérese

$$
C=D_{q}\left(h(X)+D_{q} g(X)\right)\left(E_{q}\right)^{-1} .
$$

Recuérdese también que $C$ es una $(0,2)$-matriz banda. Realizando cálculos sencillos es fácil obtener algunas de sus entradas

$$
C=\left(\begin{array}{ccccc}
0 & 0 & 0 & 0 & \cdots \\
h_{0} & \frac{h_{1}}{q} & 0 & 0 & \cdots \\
(q+1) g_{0} & \frac{(q+1)\left(g_{1}+h_{0}\right)}{q} & \frac{(q+1)\left(g_{2}+h_{1}\right)}{q^{2}} & 0 & \ldots \\
0 & \frac{(q+1)\left(q^{2}+q+1\right) g_{0}}{q} & \frac{\left(q^{2}+q+1\right)\left(h_{0}+(q+1) g_{1}\right)}{q^{2}} & \frac{\left(q^{2}+q+1\right)\left(h_{1}+(q+1) g_{2}\right)}{q^{3}} & \ldots \\
0 & 0 & \frac{\left(q^{3}+q^{2}+q+1\right)\left(q^{2}+q+1\right) g_{0}}{q^{2}} & \frac{\left(q^{3}+q^{2}+q+1\right)\left(h_{0}+\left(q^{2}+q+1\right) g_{1}\right)}{q^{3}} & \ldots \\
\vdots & \vdots & \vdots & \vdots & \ddots
\end{array}\right)
$$

Sea $\Lambda$ una matriz diagonal de índice cero cuya entrada $(k, k)$, denotada por $\lambda_{k}$, es igual a la entrada $(k, k)$ de $C$, para $k \geq 0$. Es fácil observar que

$$
\Lambda=D_{q}\left(h_{1} X+g_{2} D_{q} X^{2}\right) E_{q}^{-1} .
$$

La matriz $\Lambda$ tiene la forma siguiente

$$
\Lambda=\left(\begin{array}{cccccc}
0 & 0 & 0 & 0 & 0 & \cdots \\
0 & \frac{h_{1}}{q} & 0 & 0 & 0 & \cdots \\
0 & 0 & \frac{(q+1)\left(g_{2}+h_{1}\right)}{q^{2}} & 0 & 0 & \cdots \\
0 & 0 & 0 & \frac{\left(q^{2}+q+1\right)\left(h_{1}+(q+1) g_{2}\right)}{q^{3}} & 0 & \cdots \\
0 & 0 & 0 & 0 & \frac{\left(q^{3}+q^{2}+q+1\right)\left(h_{1}+\left(q^{2}+q+1\right) g_{2}\right)}{q^{4}} & \cdots \\
\vdots & \vdots & \vdots & \vdots & \vdots & \ddots
\end{array}\right)
$$

Sea $A$ la matriz asociada con respecto a la base monomial de una sucesión de polinomios ortogonales mónicos $\left\{p_{k}\right\}_{k \geq 0}$ con respecto a un funcional regular $\mu$. Se sabe que $\left\{p_{k}\right\}_{k \geq 0}$ es una sucesión de polinomios ortogonales $q$-clásicos si $A$ satisface la ecuación matricial

$$
A C=\Lambda A,
$$

para algún par de polinomios $h$ y $g$. La ecuación matricial (4.1) es equivalente a la ecuación en $q$-diferencias

$$
h(x) \mathcal{D}_{q} p_{k}(x)+g(x) \mathcal{D}_{q}^{2} p_{k}(x)=\lambda_{k} p_{k}(q x), \quad k \geq 0
$$


Esta forma de escribir la ecuación en diferencias (4.2) es la misma que se utiliza en el Capítulo 10 de [14]. Si $A$ es la matriz de coeficientes asociada a una sucesión ortogonal $q$-clásica entonces $L=A X A^{-1}$ es la matriz tridiagonal en $\mathcal{T}$ cuyas entradas son los coeficientes de la relación de recurrencia de tres términos satisfecha por la sucesión polinomial.

Teorema 4.1 Sea $\left\{p_{k}\right\}_{\geq 0}$ una sucesión polinomial ortogonal $q$-clásica con respecto a un funcional regular $\mu$ y sea $A$ su matriz asociada. Entonces la sucesión de momentos $\mu_{k}$ satisface la relación de recurrencia

$$
\left(h_{1}+g_{2}[k]\right) \mu_{k+1}+\left(h_{0}+g_{1}[k]\right) q \mu_{k}+g_{0}[k] q^{2} \mu_{k-1}=0, \quad k \geq 1,
$$

donde $\mu_{0}=1, \mu_{1}=h_{0} q / h_{1}$ y $h_{0}, h_{1}, g_{0}, g_{1}, g_{2}$ son los coeficientes de los polinomios $h$ y $g$ para los cuales se satisface la ecuación $A C=\Lambda A$.

Demostración. Un cálculo directo permite obtener las expresiones siguientes para las entradas de la matriz $C$

$$
\begin{aligned}
C_{k, k}=\lambda_{k} & =\frac{[k]\left(h_{1}+g_{2}[k-1]\right)}{q^{k}}, \quad k \geq 0, \\
C_{k, k-1} & =\frac{[k]\left(h_{0}+g_{1}[k-1]\right)}{q^{k-1}}, \quad k \geq 1, \\
C_{k, k-2} & =\frac{[k][k-1] g_{0}}{q^{k-2}}, \quad k \geq 2 .
\end{aligned}
$$

De la ecuación matricial $A C=\Lambda A$ se obtiene $C A^{-1}=A^{-1} \Lambda$. Recuérdese que la entrada $(k, 0)$ de $A^{-1}$ es igual al momento $\mu_{k}$, para $k \geq 0$. Como $\lambda_{0}=0$ todas las entradas en la 0-ésima columna de $A^{-1} \Lambda$ son iguales a cero. Por lo tanto, para $k \geq 0$ la entrada $(k, 0)$ de $C A^{-1}$ es cero, es decir, $\lambda_{0} \mu_{0}=0, C_{1,0} \mu_{0}+C_{1,1} \mu_{1}=0, \mathrm{y}$

$$
C_{k, k-2} \mu_{k-2}+C_{k, k-1} \mu_{k-1}+C_{k, k} \mu_{k}=0, \quad k \geq 2 .
$$

Usando las fórmulas explícitas para las entradas de $C$ la ecuación $C_{1,0} \mu_{0}+C_{1,1} \mu_{1}=0$ se convierte en $h_{0}+h_{1} q^{-1} \mu_{1}=0$, lo que implica $\mu_{1}=-h_{0} q / h_{1}$, y (4.4) se convierte en

$$
\frac{[k][k-1] g_{0}}{q^{k-2}} \mu_{k-2}+\frac{[k]\left(h_{0}+g_{1}[k-1]\right)}{q^{k-1}} \mu_{k-1}+\frac{[k]\left(h_{1}+g_{2}[k-1]\right)}{q^{k}} \mu_{k}=0, \quad k \geq 2,
$$

la cual es equivalente a la relación de recurrencia (4.3).

El método utilizado en la demostración del Teorema 4.1 puede utilizarse para obtener una relación de recurrencia para los momentos de las sucesiones polinomiales ortogonales clásicas en la clase general de Hahn, que son las sucesiones que satisfacen una ecuación en diferencias de segundo orden con el operador de Hahn $D_{q, \omega}$ en lugar de $D_{q}$. Véase [17], donde dicha relación de recurrencia se obtiene utilizando dualidad y ecuaciones funcionales satisfechas por el funcional de momentos. Nuestra recurrencia no coincide con la obtenida tomando $\omega=0$ en la recurrencia del Lema 3.2 de [17] porque ahí consideran una ecuación en diferencias que no coincide con (4.2) cuando $\omega=0$.

De la misma forma que se obtuvo (4.4) de $C A^{-1}=A^{-1} \Lambda$, ahora de $A C=\Lambda A$ se obtiene

$$
a_{k, j}\left(C_{k, k}-C_{j, j}\right)=a_{k, j+1} C_{j+1, j}+a_{k, j+2} C_{j+2, j}, \quad k>j \geq 0 .
$$


Nótese que se puede escribir (4.3) en la forma

$$
\mu_{k+1}=-\frac{\left(h_{0}+g_{1}[k]\right) q \mu_{k}+g_{0}[k] q^{2} \mu_{k-1}}{\left(h_{1}+g_{2}[k]\right)}, \quad k \geq 1 .
$$

Esta relación de recurrencia, con los valores iniciales $\mu_{0}=1$ y $\mu_{1}=-h_{0} q / h_{1}$, determina de manera única la sucesión de momentos normalizados en términos de los coeficientes de los polinomios $h$ y $g$ de (4.1) y (4.2).

Una condición necesaria para que el par $h, g$ determine, mediante la ecuación (4.1), una matriz $A$ de una sucesión polinomial $q$-ortogonal con respecto a un funcional regular $\mu$ es que $L=A X A^{-1}$ sea un elemento de $\mathcal{T}$, y esto requiere que todos los elementos de $L$ en la diagonal de índice 1 deben ser distintos de cero, esto es, $\alpha_{k} \neq 0$ para $k \geq 1$.

Es conveniente expresar los coeficientes de $h$ y $g$ en términos de los parámetros $t, u, v, y, z$, como sigue

$$
h_{0}=u-v, \quad h_{1}=q(z-y), \quad g_{0}=t(q-1), \quad g_{1}=u(q-1), \quad g_{2}=-y q(q-1) .
$$

En términos de estos nuevos parámetros la relación de recurrencia (4.6) se convierte en

$$
\mu_{k+1}=\frac{u q^{k}-v}{y q^{k}-z} \mu_{k}+\frac{t q\left(q^{k}-1\right)}{y q^{k}-z} \mu_{k-1}, \quad k \geq 1 .
$$

Un cálculo directo permite obtener la matriz de coeficientes de la relación de recurrencia de tres términos, ya que $L=A X A^{-1}$.

Teorema 4.2 Las entradas de la matriz $L=A X A^{-1}$ están dadas por las fórmulas explícitas

$$
\begin{aligned}
& \alpha_{k}=q^{k}\left(q^{k}-1\right)\left(y q^{k-2}-z\right)\left(\frac{q^{k-2}\left(u q^{k-1}-v\right)\left(v y q^{k-1}-u z\right)+t\left(y q^{2 k-2}-z\right)^{2}}{\left(y q^{2 k-3}-z\right)\left(y q^{2 k-2}-z\right)^{2}\left(y q^{2 k-1}-z\right)}\right), \\
& \sigma_{k}=[k+1] \frac{u q^{k}-v}{y q^{2 k}-z}, \quad k \geq 0,
\end{aligned}
$$

donde $\sigma_{k}=\beta_{0}+\beta_{1}+\cdots+\beta_{k}$ para $k \geq 0$, y por tanto $\beta_{k}=\sigma_{k}-\sigma_{k-1}$.

\subsection{Familias de sucesiones $q$-ortogonales con sucesiones simples de momentos}

En esta Sección se construirán varios ejemplos de familias de sucesiones de polinomios ortogonales $q$-clásicos que tienen sucesiones simples de momentos. Para cada ejemplo se encontrarán fórmulas explícitas para los momentos y los coeficientes de la relación de recurrencia de tres términos, en términos de cinco parámetros: $t, u, v, y, z$. Dichos parámetros determinan, mediante (4.7), los coeficientes de los polinomios $h$ y $g$ de la ecuación en $q$-diferencias (4.2).

De la ecuación (4.8) se pueden encontrar condiciones sobre los parámetros $t, u, v, y, z$ que simplifiquen la relación de recurrencia y permita encontrar fórmulas explícitas para los momentos. Se considera la familia de sucesiones $q$-ortogonales que satisfacen (4.2) con un polinomio $g$ tal que $g(0)=0$, es decir, $g_{0}=0$, lo que implica que $t=0$. Cuando $t=0$, la matriz $C$ es bidiagonal y entonces la 
relación (4.5) se reduce a una relación de recurrencia de dos términos para los elementos de $A$. Esta familia fue estudiada por Maroni y Mejri en [22], quienes la llamaron la familia de los polinomios ortogonales clásicos de $I(q, \omega)$.

Si $t=0$ entonces (4.8) se convierte

$$
\mu_{k+1}=\frac{u q^{k}-v}{y q^{k}-z} \mu_{k}, \quad k \geq 0, \quad \mu_{0}=1
$$

Esta relación de recurrencia de dos términos produce inmediatamente

$$
\mu_{m}=\prod_{k=0}^{m-1} \frac{u q^{k}-v}{y q^{k}-z}, \quad m \geq 1 .
$$

La ecuación (4.9) con $t=0$ se convierte en

$$
\alpha_{k}=\frac{q^{2 k-2}\left(q^{k}-1\right)\left(y q^{k-2}-z\right)\left(u q^{k-1}-v\right)\left(v y q^{k-1}-u z\right)}{\left(y q^{2 k-3}-z\right)\left(y q^{2 k-2}-z\right)^{2}\left(y q^{2 k-1}-z\right)}, \quad k \geq 1,
$$

y como las sumas de las $\sigma_{k}$ son independientes de $t$, la ecuación (4.10) se cumple cuando $t=0$.

Con el propósito de tener $\alpha_{k} \neq 0$ para $k \geq 1$, se observa de (4.12) que los parámetros $u, v, y, z$ deben satisfacer las condiciones

$$
u z-v y \neq 0, \quad z \neq y q^{m}, \quad v \neq u q^{m}, \quad u z \neq v y q^{m}, \quad m \geq 0 .
$$

Obsérvese que los momentos no cambian si los parámetros $u, v, y, z$ se multiplican por una constante distinta de cero. Nótese también que reemplazar $q$ por $1 / q$ en (4.11) es equivalente a intercambiar $u$ con $v$ y $y$ con $z$.

La expresión explícita para los coeficientes de recurrencia $\alpha_{k}$ dada por (4.12) es similar a la expresión correspondiente en la relación de recurrencia normalizada (14.15.4) para los polinomios q-Krawtchouk en el libro [14, pág. 497].

Ahora se construirán algunos ejemplos interesantes de subconjuntos de la familia descrita anteriormente, dando algunos valores apropiados a los parámetros $u, v, y, z$.

EJEMPLO 4.1 Tomando $y=u q, z=v, \mathrm{y} t=0$. Se obtiene

$$
\begin{aligned}
\mu_{m} & =\frac{u-v}{u q^{m}-v}, \quad m \geq 0, \\
\alpha_{m} & =\frac{u v q^{2 m-2}\left(q^{m}-1\right)^{2}\left(u q^{m-1}-v\right)^{2}}{\left(u q^{2 m-2}-v\right)\left(u q^{2 m-1}-v\right)^{2}\left(u q^{2 m}-v\right)}, \quad m \geq 1, \\
\sigma_{m} & =\frac{\left(q^{m+1}-1\right)\left(u q^{m}-v\right)}{(q-1)\left(u q^{2 m+1}-v\right)}, \quad m \geq 0 .
\end{aligned}
$$

Estas fórmulas para $\alpha_{m}$ y $\sigma_{m}$ coinciden con las fórmulas correspondientes obtenidas de (14.15.4) de [14, pág. 497], cuando se toman los parámetros $p=-u / v$ y $N=-1$. Obsérvese que para los polinomios $q$-Krawtchouk habituales, el parámetro $N$ es un entero positivo y las sucesiones polinomiales son finitas. 
Haciendo $u=q$ y $v=1$ entonces se obtiene un elemento particular en esta familia y las ecuaciones (4.13), (4.14), (4.15) se convierten respectivamente,

$$
\begin{aligned}
& \mu_{m}=\frac{1}{[m+1]}, \quad m \geq 0, \\
& \alpha_{m}=\frac{q^{2 m-1}\left(q^{m}-1\right)^{4}}{\left(q^{2 m-1}-1\right)\left(q^{2 m}-1\right)^{2}\left(q^{2 m+1}-1\right)}=\frac{q^{2 m-1}[m]^{4}}{[2 m-1][2 m]^{2}[2 m+1]}, \quad m \geq 1, \\
& \sigma_{m}=\frac{\left(q^{m+1}-1\right)^{2}}{(q-1)\left(q^{2 m+2}-1\right)}=\frac{[m+1]^{2}}{[2 m+2]}, \quad m \geq 0 .
\end{aligned}
$$

El Ejemplo 4.1 se generaliza de la siguiente manera.

EJEMPlo 4.2 Sea $z=v, t=0$ y $y=u q^{r}$, donde $r$ es un entero positivo. Entonces se tiene

$$
\mu_{m}=\frac{(u-v)(u q-v)\left(u q^{2}-v\right) \cdots\left(u q^{r-1}-v\right)}{\left(u q^{m}-v\right)\left(u q^{m+1}-v\right) \cdots\left(u q^{m+r-1}-v\right)}, \quad m \geq 1 .
$$

Obsérvese que el numerador no depende de $m$ y el número de factores en el denominador es $r$.

En este caso, los coeficientes de la relación de recurrencia son

$$
\begin{aligned}
& \alpha_{m}=\frac{u v q^{2 m-2}\left(q^{m}-1\right)\left(u q^{m-1}-v\right)\left(q^{m+r-1}-1\right)\left(q^{m+r-2}-v\right)}{\left(u q^{2 m+r-3}-v\right)\left(u q^{2 m+r-2}-v\right)^{2}\left(u q^{2 m+r-1}-v\right)}, \quad m \geq 1, \\
& \sigma_{m}=\frac{\left(q^{m+1}-1\right)\left(u q^{m}-v\right)}{(q-1)\left(u q^{2 m+r}-v\right)}, \quad m \geq 0 .
\end{aligned}
$$

Éstos se obtienen de (14.15.4) de [14, pág. 497], tomando $p=-u q^{r-1} / v$ y $N=-r$. Parece que tales polinomios $q$-Krawtchouk no estándar, no han sido considerados en la literatura de polinomios ortogonales.

Un ejemplo particular en esta familia, con $r=3, u=q$, y $v=1$, tiene momentos y coeficientes de la relación de recurrencia dados por

$$
\begin{aligned}
& \mu_{m}=\frac{(q-1)\left(q^{2}-1\right)\left(q^{3}-1\right)}{\left(q^{m+1}-1\right)\left(q^{m+2}-1\right)\left(q^{m+3}-1\right)}=\frac{[1][2][3]}{[m+1][m+2][m+3]}, \quad m \geq 0, \\
& \alpha_{m}=\frac{q^{2 m-1}\left(q^{m}-1\right)^{2}\left(q^{m+2}-1\right)^{2}}{\left(q^{2 m+1}-1\right)\left(q^{2 m+2}-1\right)^{2}\left(q^{2 m+3}-1\right)}=\frac{q^{2 m-1}[m]^{2}[m+2]^{2}}{[2 m+1][2 m+2]^{2}[2 m+3]}, \quad m \geq 1, \\
& \sigma_{m}=\frac{\left(q^{m+1}-1\right)^{2}}{(q-1)\left(q^{2 m+4}-1\right)}=\frac{[m+1]^{2}}{[2 m+4]}, \quad m \geq 0 .
\end{aligned}
$$

EjEMPLo 4.3 Si $t=0, v=z$ y $u=q^{r} y$ para algún entero no negativo $r$ entonces se obtiene una familia de polinomios $q$-ortogonales tales que $\alpha_{r+1}=0$ y todos los otros coeficientes $\alpha_{k}$ son distintos de cero. En este caso se obtiene una sucesión finita de polinomios $q$-ortogonales con momentos 
simples para los cuales

$$
\begin{aligned}
& \mu_{m}=\frac{\left(y q^{m}-z\right)\left(y q^{m+1}-z\right) \cdots\left(y q^{m+r-1}-z\right)}{(y-z)(y q-z) \cdots\left(y q^{r-1}-z\right)}, \quad m \geq 0, \\
& \alpha_{m}=\frac{y z q^{2 m-2}\left(q^{m}-1\right)\left(y q^{m-2}-z\right)\left(y q^{m-1+r}-z\right)\left(q^{m-1}-q^{r}\right)}{\left(q^{2 m-1} y-z\right)\left(q^{2 m-2} y-z\right)^{2}\left(q^{2 m-3} y-z\right)}, \quad 1 \leq m \leq r, \\
& \sigma_{m}=\frac{\left(q^{m+1}-1\right)\left(y q^{m+r}-z\right)}{(q-1)\left(y q^{2 m}-z\right)}, \quad m \geq 0 .
\end{aligned}
$$

Obsérvese que el factor $\left(q^{m-1}-q^{r}\right)$ en (4.16) desaparece cuando $m=r+1$, lo cual implicaría $\alpha_{r+1}=0$. Cuando $1 \leq m \leq r$, estas sucesiones son las usuales sucesiones polinómicas finitas $q$ Krawtchouk.

Un ejemplo concreto y simple se obtiene tomando $u=q^{2+r}, y=q^{2}, v=1, z=1$ y $t=0$.

EJEMPLO 4.4 Si $u=0$ y $t=0$ se obtienen

$$
\begin{aligned}
& \mu_{m}=\frac{(-v)^{m}}{(y-z)(y q-z)\left(y q^{2}-z\right) \cdots\left(y q^{m-1}-z\right)}, \quad m \geq 0 \\
& \alpha_{m}=-\frac{y v^{2} q^{3 m-3}\left(q^{m}-1\right)\left(y q^{m-2}-z\right)}{\left(y q^{2 m-3}-z\right)\left(y q^{2 m-2}-z\right)^{2}\left(y q^{2 m-1}-z\right)}, \quad m \geq 1, \\
& \sigma_{m}=-\frac{v\left(q^{m+1}-1\right)}{(q-1)\left(y q^{2 m}-z\right)}=-\frac{v[m]}{y q^{2 m}-z}, \quad m \geq 0 .
\end{aligned}
$$

Los coeficientes de recurrencia dados en (4.17) y (4.18) coinciden con los obtenidos de (14.22.4) en [14, pág. 527], cuando el parámetro $a$ es igual a $-y /(z q)$ y tomando $v=z$. Por lo tanto, las sucesiones del Ejemplo 4.3 incluyen los polinomios $q$-Bessel.

Tomando ahora $y=q, z=1, v=1-q, u=0$ y $t=0$ se obtiene un ejemplo con momentos muy simples. Esto es, los momentos y los coeficientes de la recurrencia son

$$
\begin{aligned}
& \mu_{m}=\frac{1}{[m] !}, \quad m \geq 0, \\
& \alpha_{m}=-\frac{q^{3 m-2}(q-1)^{2}\left(q^{m-1}-1\right)\left(q^{m}-1\right)}{\left(q^{2 m-2}-1\right)\left(q^{2 m-1}-1\right)^{2}\left(q^{2 m}-1\right)}=-\frac{q^{3 m-2}[m-1][m]}{[2 m-2][2 m-1]^{2}[2 m]}, \quad m \geq 1, \\
& \sigma_{m}=\frac{q^{m+1}-1}{q^{2 m+1}-1}=\frac{[m+1]}{[2 m+1]}, \quad m \geq 0 .
\end{aligned}
$$

Obsérvese que en (4.19) $\alpha_{1}=-\frac{q}{1+q}$. 
Si en lugar de $y=q$ se toma $y=q^{r}$, donde $r$ es un entero positivo, se obtiene

$$
\begin{aligned}
& \mu_{m}=\frac{1}{[r][r+1] \cdots[r+m-1]}, \quad m \geq 1, \\
& \alpha_{m}=-\frac{q^{3 m+r-3}[m+r-2][m]}{[2 m+r-3][2 m+r-2]^{2}[2 m+r-1]}, \quad m \geq 1, \\
& \sigma_{m}=\frac{[m+1]}{[2 m+r]}, \quad m \geq 0 .
\end{aligned}
$$

EJEMPLO 4.5 Sean $y=0$ y $t=0$. Entonces (4.10), (4.11) y (4.12) permiten obtener

$$
\begin{aligned}
& \mu_{m}=(-z)^{-m}(u-v)(u q-v)\left(u q^{2}-v\right) \cdots\left(u q^{m-1}-v\right), \quad m \geq 1, \\
& \alpha_{m}=u z^{-2} q^{2 m-2}\left(q^{m}-1\right)\left(u q^{m-1}-v\right), \quad m \geq 1, \\
& \sigma_{m}=-[m+1] z^{-1}\left(u q^{m}-v\right), \quad m \geq 0 .
\end{aligned}
$$

Estos coeficientes de recurrencia corresponden a los polinomios $q$-Laguerre/Wall pequeños. Véase (14.20.4) en [14, pág. 519].

Para el caso particular con $y=0, t=0, u=q, v=1$ y $z=1-q$, se tiene

$$
\mu_{m}=[m] !, \quad \alpha_{m}=q^{2 m-1}[m]^{2}, \quad \sigma_{m}=[m+1]^{2} .
$$

Este ejemplo es generalizado de la siguiente manera. Tomando $y=0, t=0, v=1, z=1-q \mathrm{y}$ $u=q^{r}$ para el mismo entero positivo $r$, entonces se obtiene

$$
\begin{aligned}
& \mu_{m}=[r][r+1] \cdots[r+m-1], \quad m \geq 1, \\
& \alpha_{m}=[m][m+r-1] q^{2 m+r-2}, \quad m \geq 1, \\
& \sigma_{m}=[m+1][m+r], \quad m \geq 0 .
\end{aligned}
$$

\subsection{Otras familias de polinomios $q$-ortogonales con momentos sim- ples}

En esta Sección se consideran familias de polinomios $q$-ortogonales que satisfacen la ecuación en $q$-diferencias (4.2) con los polinomios $h$ y $g$ tal que los coeficientes $h_{0}$ y $g_{1}$ son iguales a cero.

Sean $h_{0}=g_{1}=0$, en este caso, la relación de recurrencia (4.6) se convierte en

$$
\mu_{k+1}=-\frac{g_{0}[k] q^{2}}{h_{1}+g_{2}[k]} \mu_{k-1}, \quad k \geq 1 .
$$

En el caso general se tiene $\mu_{1}=-h_{0} q / h_{1}$, y entonces $\mu_{1}=0$ si $h_{0}=0$. Por lo tanto, por la recurrencia (4.20) todos los momentos $\mu_{n}$ con $n$ impar son iguales a cero, y

$$
\mu_{2 m}=(-1)^{m} g_{0}^{m} q^{2 m} \prod_{k=1}^{m} \frac{[2 k-1]}{h_{1}+g_{2}[2 k-1]}, \quad m \geq 1 .
$$


En este caso (4.7) se convierte en

$$
h_{0}=0, \quad h_{1}=q(z-y), \quad g_{0}=t(q-1), \quad g_{1}=0, \quad g_{2}=-y q(q-1) .
$$

Obsérvese que $u=0$ y $v=0$.

EJEMPLO 4.6 En términos de los parámetros $t, y, z$ la ecuación (4.21) se convierte en

$$
\mu_{2 m}=\frac{t^{m} q^{m}(q-1)\left(q^{3}-1\right) \cdots\left(q^{2 m-1}-1\right)}{(y q-z)\left(y q^{3}-z\right) \cdots\left(y q^{2 m-1}-z\right)}, \quad m \geq 1 .
$$

Los coeficientes de recurrencia para este caso son:

$$
\alpha_{m}=\frac{t q^{m}\left(q^{m}-1\right)\left(y q^{m-2}-z\right)}{\left(y q^{2 m-3}-z\right)\left(y q^{2 m-1}-z\right)}, \quad m \geq 1,
$$

y, como $u=0$ y $v=0$, se observa de la ecuación (4.10) que todos los $\sigma_{m}$ son cero.

Los coeficientes de recurrencia (4.22) y $\sigma_{m}=0$ se obtienen cuando se toman $a=b$ y $c=-b$ en la relación de recurrencia normalizada (14.5.4) para los polinomios $q$-Jacobi grandes en [14, pág. 439], y entonces se hace $b=y /\left(z q^{2}\right)$.

Un caso concreto y simple se obtiene cuando $y=q^{2}, z=1$ y $t=q^{-1}$. Con esta elección de los parámetros se obtiene

$$
\mu_{2 m}=\frac{1}{[2 m+1]}, \quad m \geq 0
$$

y

$$
\alpha_{m}=\frac{q^{m-1}[m]^{2}}{[2 m-1][2 m+1]}, \quad m \geq 1 .
$$

A continuación se generaliza el Ejemplo 4.6.

EJEMPLO 4.7 Haciendo $z=1, t=q^{-1}$ y $y=q^{2 r}$, donde $r$ es un entero positivo. Se obtiene

$$
\mu_{2 m}=\frac{[1][3][5] \cdots[2 m-1]}{[2 r+1][2 r+3] \cdots[2 r+2 m-1]}, \quad m \geq 1
$$

y

$$
\alpha_{m}=\frac{q^{m-1}[m][m+2 r-2]}{[2 m+2 r-3][2 m+2 r-1]}, \quad m \geq 1
$$

Si se permite que $r$ sea un entero negativo, entonces sólo se obtiene una sucesión finita de polinomios $q$-ortogonales, ya que en tal caso (4.23) da $\alpha_{-2 r+2}=0$. Obsérvese que el denominador de (4.23) no desaparece cuando $r$ es negativo.

Un ejemplo particular simple se obtiene cuando se toman $y=0, z=1-q$ y $t=q^{-1}$. En este caso (4.21) y (4.22) se convierten respectivamente en

$$
\begin{aligned}
\mu_{2 m} & =[1][3][5] \cdots[2 m-1], \quad m \geq 1, \\
\alpha_{m} & =q^{m-1}[m], \quad m \geq 1 .
\end{aligned}
$$


En [25] y [26] los autores encontraron los momentos de todos los polinomios ortogonales clásicos. Para los polinomios ortogonales q-clásicos los momentos se presentan en los teoremas 38 y 40 de [26], donde se puede observar que en la mayoría de los casos, las fórmulas de los momentos no son simples e implican varios conjuntos diferentes de parámetros. Sería difícil utilizar tales fórmulas para encontrar los casos en que los momentos tienen formas simples. Los polinomios ortogonales $q$ clásicos han sido ampliamente estudiados por muchos autores, utilizando diversos enfoques. Véase, por ejemplo, [3], [13], [17], [23], [24] y [36]. Algunos resultados interesantes sobre los momentos se presentan en [9]. El libro [14] presenta un estudio muy detallado de las sucesiones $q$-polinomiales. Una de las familias grandes que se considera en este Capítulo fue estudiada en [22] utilizando el enfoque de dualidad de Maroni. 


\section{CAPÍTULO 5 | \\ LREPRESENTACIÓN DE MATRICES DOBLEMENTE INFINITAS COMO SERIES DE LAURENT NO-CONMUTATIVAS}

El objetivo de este Capítulo es extender el reciente desarrollo sobre la teoría de matrices infinitas a matrices doblemente infinitas, presentada en [5]. Esto se traduce en que los problemas analíticos que aparecen en la multiplicación de matrices infinitas, y la continuidad de los operadores representados por tales matrices se estudiaron ampliamente, pero el estudio de las propiedades puramente algebraicas de las matrices infinitas no ha recibido la atención que se merece. Los espacios de matrices infinitas triangulares son los que se han estudiado con más detalle y se utilizan a menudo en combinatoria y algunas ramas del álgebra. Por otro lado, la teoría de las matrices doblemente infinitas $\left[a_{n, k}\right]$, donde los índices corren sobre todos los enteros, está subdesarrollada. Sólo se han estudiado algunos tipos particulares de matrices bi-infinitas, tales como las matrices Toeplitz por bloques y las matrices tridiagonales de Jacobi.

Algunos anillos de matrices doblemente infinitas libres de convergencia fueron estudiados por Köthe y Toeplitz en [15]. Jabotinky en [12] representó operadores de composición por medio de matrices doblemente infinitas para estudiar la iteración de funciones analíticas. Barnabei, Brini y Nicoletti estudiaron las propiedades de recurrencia y las conexiones con el cálculo umbral de matrices doblemente infinitas en [6]. Más recientemente, varios autores han extendido los grupos de Riordan, que son generados por operadores de multiplicación y composición, al contexto bi-infinito. Véanse [19] y [20].

Las matrices de Toeplitz bi-infinitas por bloques se han estudiado durante mucho tiempo. Véase [31] y las referencias en el mismo. En [33] se utilizan matrices doblemente infinitas para obtener resultados sobre grupos de matrices de Pascal generalizadas. Una versión multimatriz de matrices doblemente infinitas se utilizó en [32] para estudiar las fórmulas de inversión de Lagrange. Una buena referencia para la teoría de las series de potencias formales y las series de Laurent formales es el libro de Henrici [11].

El esquema del Capítulo es como sigue: en la Sección 5.1 se introduce una nueva forma de tratar con matrices doblemente infinitas y se muestra que es una forma conveniente y efectiva de obtener propiedades algebraicas de dichas matrices. Se presentan las definiciones básicas y la notación que se utilizará en este Capítulo. La idea básica es bastante simple: se considera una matriz doblemente infinita como la suma de sus submatrices diagonales. Tal idea fue motivada por el trabajo con el 
enfoque matricial de polinomios ortogonales introducido en [34], donde se consideran ecuaciones en matrices infinitas que se resolvieron al encontrar las diagonales de las matrices desconocidas de forma recursiva. Los resultados obtenidos para matrices doblemente infinitas arrojan inmediatamente algunos resultados relacionados para matrices infinitas con índices en los enteros no negativos, por medio de descomposición y truncamiento de bloques. Por ejemplo, algunos resultados en [35] se pueden obtener de esta manera.

En la Sección 5.2 se da la representación por diagonales de las matrices doblemente infinitas de Hessenberg, lo que permite considerar el anillo de las matrices doblemente infinitas de Hesseberg como un anillo de series de Laurent en términos de un símbolo indeterminado $S$ (no es una variable, sólo nos ayuda a representar las series de Laurent no-conmutativas) con coeficientes que no conmutan con $S$. También se muestra cómo usar la representación diagonal para calcular multiplicaciones de matrices y para encontrar inversas de algunas matrices. En la Sección 5.3 se utiliza la representación diagonal para obtener algunas propiedades relacionadas con la similaridad y la conmutatividad de las matrices doblemente infinitas.

\subsection{Matrices doblemente infinitas}

En esta Sección se da la definición de anillo libre de convergencia de matrices doblemente infinitas, se presentan definiciones de ciertos conjuntos de matrices diagonales y la notación que será utilizada en este Capítulo. Cabe mencionar que es un desarrollo parecido a las series formales de Laurent, la diferencia está en que se reemplazan los coeficientes de la serie por matrices diagonales.

Sea $R$ un anillo asociativo con unidad, denotada por $e$.

Definición 5.1 Sea $\mathfrak{L}$ el conjunto de todas las matrices bilateralmente infinitas $A=\left[a_{n, k}\right]$, donde los índices $n$ y $k$ corren sobre todos los enteros $y$, o bien $A=0$ o existe un número entero $i(A)$, llamado indice de A, tal que

$$
i(A)=\min \left\{n-k:(n, k) \in \mathbb{Z} \times \mathbb{Z}, a_{n, k} \neq 0\right\} .
$$

Esto significa que el soporte de la función $(n, k) \rightarrow a_{n, k}$, de $\mathbb{Z} \times \mathbb{Z}$ a $R$, está contenido en el subespacio

$$
\{(n, k) \in \mathbb{Z} \times \mathbb{Z}: n-k \geq i(A)\}
$$

Se dice que las entradas $a_{n, k}$ están en la diagonal de indice $j$ si $n-k=j$. Una matriz es diagonal si todas sus entradas distintas de cero, si las hay, se encuentran en una sola diagonal.

Definición 5.2 Sea $\mathcal{D}_{m}$ el conjunto de matrices diagonales de indice $m$, para $m \in \mathbb{Z}$. La matriz cero está en $\mathcal{D}_{m}$ para cada $m$.

Las matrices en $\mathfrak{L}$ están representadas de la manera habitual. El primer subíndice corresponde a filas y aumenta a medida que avanzamos hacia abajo, y el segundo subíndice corresponde a columnas y éste aumenta a medida que avanzamos hacia la derecha. 
EJEMPLo 5.1 La matriz $A$ es una matriz doblemente infinita con $i(A)=-1$

$$
A=\left(\begin{array}{cccc|ccccc}
\ddots & \vdots & \vdots & \vdots & \vdots & \vdots & \vdots & \vdots & \\
\cdots & a_{-4-3} & 0 & 0 & 0 & 0 & 0 & 0 & \cdots \\
\cdots & a_{-3-3} & a_{-3-2} & 0 & 0 & 0 & 0 & 0 & \cdots \\
\cdots & a_{-2-3} & a_{-2-2} & a_{-2-1} & 0 & 0 & 0 & 0 & \cdots \\
\cdots & a_{-1-3} & a_{-1-2} & a_{-1-1} & a_{-10} & 0 & 0 & 0 & \cdots \\
- & - & - & - & - & - & - & - & - \\
\cdots & a_{0-3} & a_{0-2} & a_{0-1} & a_{00} & a_{01} & 0 & 0 & \cdots \\
\cdots & a_{1-3} & a_{1-2} & a_{1-1} & a_{10} & a_{11} & a_{12} & 0 & \cdots \\
& \vdots & \vdots & \vdots & \vdots & \vdots & \vdots & \vdots & \ddots
\end{array}\right)
$$

Además, si $A$ es un elemento distinto de cero de $\mathfrak{L}$, entonces todas las entradas $a_{n, k}$ arriba de la diagonal de índice $i(A)$ son cero y hay al menos una entrada distinta de cero en la diagonal de índice $i(A)$. Decimos que $\mathfrak{L}$ es el conjunto de semi-matrices inferiores, o bien, también se llaman matrices de Hessenberg infinitas. Es fácil verificar que $\mathfrak{L}$ es un anillo con las operaciones usuales de suma y multiplicación.

Si $A=\left(a_{n, k}\right)$ y $B=\left(b_{n, k}\right)$ son elementos de $\mathfrak{L}$ y $A B=C=\left(c_{n, k}\right)$ entonces se tiene

$$
c_{n, k}=\sum_{j=k+i(B)}^{n-i(A)} a_{n, j} b_{j, k}
$$

y esto muestra que las entradas $c_{n, k}$ son distintas de cero solamente cuando $n-k \geq i(A)+i(B)$, esto es, $i(C) \geq i(A)+i(B)$.

Nótese que la multiplicación de dos elementos de $\mathfrak{L}$ implica sólo sumas finitas de elementos de $R$, por lo tanto, no surgen problemas de convergencia. Köthe y Toeplitz en [15] consideraron algunos anillos libres de convergencia que son más grandes que $\mathfrak{L}$. La identidad para la multiplicación es denotada por $I$ y es el elemento de $\mathcal{D}_{0}$ tal que su entrada $(k, k)$ es igual a $e$ para cada $k \in \mathbb{Z}$.

Se muestra a continuación que los elementos de $\mathfrak{L}$ están conectados con series formales de Laurent.

Definición 5.3 Sea $\mathcal{V}$ el conjunto de vectores columna infinitos $v=\left(v_{j}\right)$ tal que $v_{j} \in R$ para cada $j \in \mathbb{Z}$ y, o $v=0$ o existe un entero $i(v)$ tal que $v_{j}=0$ si $j<i(v)$ y $v_{i(v)} \neq 0$.

Nótese que cada elemento $A$ de $\mathfrak{L}$ tiene sus columnas en $\mathcal{V}$ y que el mapeo $v \rightarrow A v$ es un mapeo aditivo bien definido en el conjunto $\mathcal{V}$, donde

$$
(A v)_{n}=\sum_{k=i(v)}^{n-i(A)} a_{n, k} v_{k}
$$

Cada elemento $v=\left(v_{j}\right)$ de $\mathcal{V}$ se puede identificar con la serie formal de Laurent

$$
v(z)=\sum_{j=i(v)}^{\infty} v_{j} z^{j},
$$


donde $z$ representa un símbolo indeterminado y los coeficientes $v_{j}$ están en $R$. Por lo tanto, $A v$ se puede identificar con la serie de Laurent

$$
A v(z)=\sum_{n \geq i(A)+i(v)}(A v)_{n} z^{n}=\sum_{n \geq i(A)+i(v)} \sum_{k=i(v)}^{n-i(A)} a_{n, k} v_{k} z^{n}
$$

y para cada $k \in \mathbb{Z}$ las series

$$
A e z^{k}=\sum_{n \geq i(A)+k} a_{n, k} z^{n}
$$

correspondientes a la $k$-ésima columna de $A$, y se puede considerar a $A$ como una sucesión doblemente infinita de series formales de Laurent con coeficientes en $R$. Está claro que las filas de los elementos de $\mathfrak{L}$ pueden considerarse series de Laurent formales invertidas. Véase [11].

Definición 5.4 Sea $S$ la matriz de desplazamiento, la cual es un elemento de $\mathcal{D}_{1}$ tal que su entrada $(k+1, k)$ es igual a $e$ para cada $k$ en $\mathbb{Z}$.

Es fácil verificar que $S$ es invertible y su inversa $S^{-1}$ es un elemento de $\mathcal{D}_{-1}$ con la entrada $(k, k+1)$ igual a $e$ por cada $k$ en $\mathbb{Z}$.

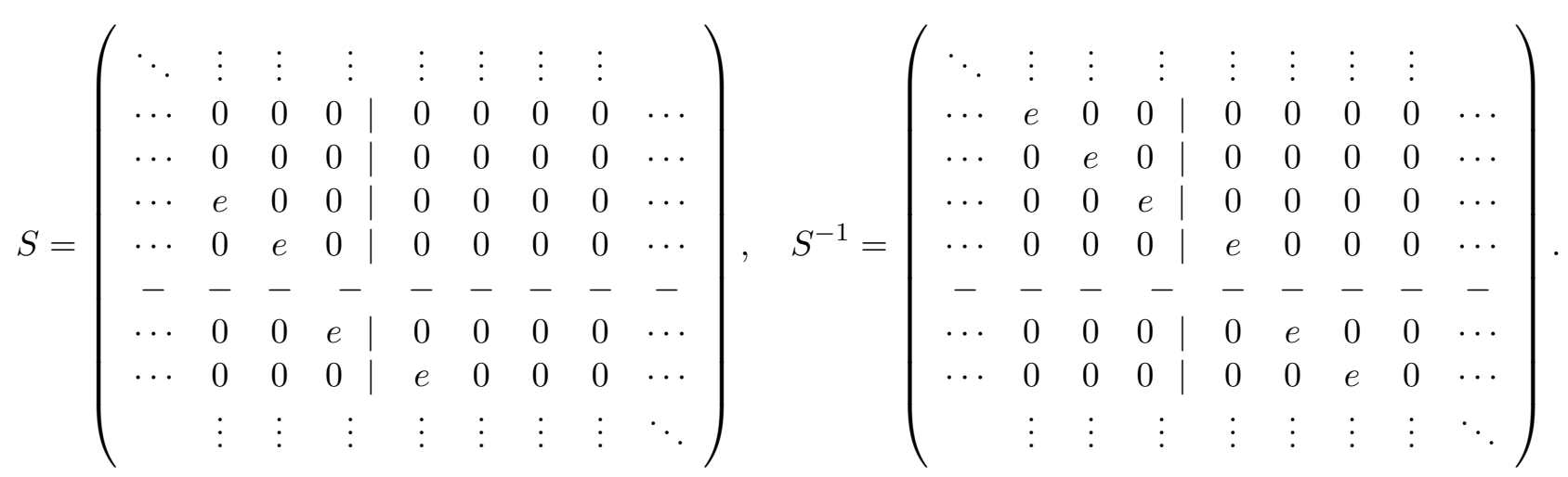

Si $A$ es un elemento de $\mathfrak{L}$ entonces $S A$ se obtiene trasladando $A$ una posición hacia abajo, $A S$ se obtiene trasladando $A$ una posición a la izquierda, $S^{-1} A$ es igual a $A$ trasladada una posición hacia arriba, y $A S^{-1}$ es igual a $A$ trasladada una posición a la derecha. Obsérvese el siguiente ejemplo.

Ejemplo 5.2 Sea $A$ la matriz del Ejemplo 5.1, entonces las matrices $S A, A S, S^{-1} A$ y $A S^{-1}$ son respectivamente de la forma:

$$
\left(\begin{array}{ccc|cccc}
\vdots & \vdots & \vdots & \vdots & \vdots & \vdots & \\
\cdots & 0 & 0 & 0 & 0 & 0 & 0 \cdots \\
\cdots a_{-4-3} & 0 & 0 & 0 & 0 & 0 & 0 \cdots \\
\cdots a_{-3-3} & a_{-3-2} & 0 & 0 & 0 & 0 & 0 \cdots \\
\cdots a_{-2-3} & a_{-2-2} & a_{-2-1} & 0 & 0 & 0 & 0 \cdots \\
- & - & - & - & - & - & - \\
\cdots a_{-1-3} & a_{-1-2} & a_{-1-1} & a_{-10} & 0 & 0 & 0 \cdots \\
\cdots a_{0-3} & a_{0-2} & a_{0-1} & a_{00} & a_{01} & 0 & 0 \cdots \\
\vdots & \vdots & \vdots & \vdots & \vdots & \vdots & \vdots
\end{array}\right),\left(\begin{array}{cccc|cccc}
\vdots & \vdots & \vdots & \vdots & \vdots & \vdots & \vdots \\
\cdots & 0 & 0 & 0 & 0 & 0 & 0 & 0 \cdots \\
\cdots a_{-3-2} & 0 & 0 & 0 & 0 & 0 & 0 \cdots \\
\cdots a_{-2-2} & a_{-2-1} & 0 & 0 & 0 & 0 & 0 \cdots \\
\cdots a_{-1-2} & a_{-1-1} & a_{-10} & 0 & 0 & 0 & 0 \ldots \\
- & - & - & - & - & - & - \\
\cdots a_{0-2} & a_{0-1} & a_{00} & a_{01} & 0 & 0 & 0 \cdots \\
\cdots a_{1-2} & a_{1-1} & a_{10} & a_{11} & a_{12} & 0 & 0 \cdots \\
\vdots & \vdots & \vdots & \vdots & \vdots & \vdots & \vdots
\end{array}\right)
$$




$$
\left(\begin{array}{ccc|cccc}
\vdots & \vdots & \vdots & \vdots & \vdots & \vdots & \\
\cdots a_{-3-3} & a_{-3-2} & 0 & 0 & 0 & 0 & 0 \cdots \\
\cdots a_{-2-3} & a_{-2-2} & a_{-2-1} & 0 & 0 & 0 & 0 \cdots \\
\cdots a_{-1-3} & a_{-1-2} & a_{-1-1} & a_{-10} & 0 & 0 & 0 \cdots \\
\cdots a_{0-3} & a_{0-2} & a_{0-1} & a_{00} & a_{01} & 0 & 0 \cdots \\
- & - & - & - & - & - & - \\
\cdots a_{1-3} & a_{1-2} & a_{1-1} & a_{10} & a_{11} & a_{12} & 0 \cdots \\
\cdots a_{2-3} & a_{2-2} & a_{2-1} & a_{20} & a_{21} & a_{22} & a_{23} \cdots \\
\vdots & \vdots & \vdots & \vdots & \vdots & \vdots & \vdots
\end{array}\right),\left(\begin{array}{ccc|ccc}
\vdots & \vdots & \vdots & \vdots & \vdots & \vdots \\
\cdots a_{-5-3} & a_{-4-3} & 0 & 0 & 0 & 0 \cdots \\
\ldots a_{-4-3} & a_{-3-3} & a_{-3-2} & 0 & 0 & 0 \cdots \\
\cdots a_{-3-3} & a_{-2-3} & a_{-2-2} & a_{-2-1} & 0 & 0 \cdots \\
\cdots a_{-2-3} & a_{-1-3} & a_{-1-2} & a_{-1-1} & a_{-10} & 0 \cdots \\
- & - & - & - & - & - \\
\cdots a_{-1-3} & a_{0-3} & a_{0-2} & a_{0-1} & a_{00} & a_{01} \cdots \\
\cdots a_{-2-3} & a_{1-3} & a_{1-2} & a_{1-1} & a_{10} & a_{11} \cdots \\
\vdots & \vdots & \vdots & \vdots & \vdots & \vdots \\
\vdots & & & & & \vdots
\end{array}\right) .
$$

Definición 5.5 Sea $S^{m} A S^{-m}$ una traslación por $m$ posiciones de $A$ en la dirección diagonal, esto es, la entrada $(n, k)$ del producto $S^{m} A S^{-m}$ es igual a $a_{n-m, k-m}$. Se denota como

$$
A^{[m]}=S^{m} A S^{-m},
$$

y se llama a $A^{[m]}$ el desplazamiento diagonal de $A$ por $m$ pasos.

Nótese que $(A B)^{[m]}=A^{[m]} B^{[m]}$.

Ejemplo 5.3 Sea $A$ la matriz del Ejemplo 5.1 y sea $m=1$, por lo tanto $A^{[1]}=S^{1} A S^{-1}$ el cual es un desplazamiento diagonal de $A$ por 1 paso, esto es,

$$
S A S^{-1}=\left(\begin{array}{cccc|ccccc}
\ddots & \vdots & \vdots & \vdots & \vdots & \vdots & \vdots & \vdots & \\
\cdots & a_{-5-4} & 0 & 0 & 0 & 0 & 0 & 0 & \cdots \\
\cdots & a_{-4-4} & a_{-4-3} & 0 & 0 & 0 & 0 & 0 & \cdots \\
\cdots & a_{-3-4} & a_{-3-3} & a_{-3-2} & 0 & 0 & 0 & 0 & \cdots \\
\cdots & a_{-2-4} & a_{-2-3} & a_{-2-2} & a_{-2-1} & 0 & 0 & 0 & \cdots \\
- & - & - & - & - & - & - & - & - \\
\cdots & a_{-1-4} & a_{-1-3} & a_{-1-2} & a_{-1-1} & a_{-10} & 0 & 0 & \cdots \\
\cdots & a_{0-4} & a_{0-3} & a_{0-2} & a_{0-1} & a_{00} & a_{01} & 0 & \cdots \\
& \vdots & \vdots & \vdots & \vdots & \vdots & \vdots & \vdots & \ddots
\end{array}\right)
$$

En la siguiente proposición, se enumeran algunas propiedades básicas de los elementos del anillo $\mathfrak{L}$. Las pruebas son cálculos simples.

Proposición 5.6 1. Si $A \in \mathfrak{L}$ conmuta con $S$ (o con $S^{-1}$ ) entonces $A$ es constante a lo largo de sus diagonales, esto es, $A$ es una matriz de Toeplitz.

2. Si $A$ conmuta con $S^{m}$ para algún $m \neq 0$ entonces $A$ es una matriz de Toeplitz por bloques de tamaño $|m| \times|m|$.

3. El conjunto $\mathcal{D}_{0}$ es cerrado bajo adición y multiplicación y contiene la identidad $I$.

4. Un elemento $U=\left[u_{n, k}\right]$ de $\mathcal{D}_{0}$ es invertible si y sólo si $u_{k, k}$ es invertible en $R$ para cada $k$ en $\mathbb{Z}$, y en este caso $U^{-1}$ está también en $\mathcal{D}_{0}$.

5. Si $W$ está en $\mathcal{D}_{m}$ entonces $W=S^{m} U$ donde $U=S^{-m} W$ y $U$ está en $\mathcal{D}_{0}$.

6. Un elemento $W$ de $\mathcal{D}_{m}$ es invertible si y sólo si su entrada $(k+m, k)$ es invertible en $R$ para cada $k$ en $\mathbb{Z}$, y en ese caso $W^{-1}$ está en $\mathcal{D}_{-m}$.

7. Si $A$ y $B$ están en $\mathfrak{L}$ y $m$ y $k$ son enteros entonces $S^{m} A S^{k} B=S^{m+k} A^{[-k]} B$. 


\subsection{Representación por diagonales y series de Laurent no-conmu- tativas}

Sea $A$ un elemento de $\mathfrak{L}$ y sea $m$ un entero. Se denota por $\delta_{m}(A)$ al elemento de $\mathcal{D}_{m}$ cuya entrada en la $(k+m, k)$ posición es el elemento $a_{k+m, k}$ de $A$ para cada $k$ en $\mathbb{Z}$, es decir, $\delta_{m}(A)$ es la matriz diagonal de indice $m$ que coincide con A sobre la $m$-ésima diagonal. Entonces se puede expresar una matriz no cero $A$ de la forma

$$
A=\sum_{k \geq i(A)} \delta_{k}(A)
$$

Esta es sólo una manera conveniente de decir que $A$ se construye colocando sus diagonales en los lugares apropiados. Obsérvese el siguiente ejemplo.

Ejemplo 5.4 Sea $A \in \mathfrak{L}$ con $i(A)=-1$, construimos $A$ utilizando a $\delta_{m}(A)$ de la siguiente manera:

$$
\begin{aligned}
A=\left(\begin{array}{ccccccc}
\ddots & \vdots & \vdots & \vdots & \vdots & \\
\cdots & a_{-2,-2} & a_{-2,-1} & 0 & 0 & \cdots \\
\cdots & a_{-1,-2} & a_{-1,-1} & a_{-1,0} & 0 & \cdots \\
\cdots & a_{0,-2} & a_{0,-1} & a_{0,0} & a_{0,1} & \cdots \\
\cdots & a_{1,-2} & a_{1,-1} & a_{1,0} & a_{1,1} & \cdots \\
\vdots & \vdots & \vdots & \vdots & \ddots
\end{array}\right)=\sum_{k \geq-1} \delta_{k}(A)=\delta_{-1}(A)+\delta_{0}(A)+\delta_{1}(A)+\delta_{2}(A)+\cdots+ \\
\\
\vdots \\
\quad
\end{aligned}
$$

Nótese que en (5.6) colocamos el elemento $(n, k)$ que corresponde a ese lugar en la diagonal de índice $n-k$, así en la suma sólo contribuye un elemento distinto de cero ya que en las posiciones restantes los elementos son cero. 
Ahora se define

$$
\gamma_{m}(A)=S^{-m} \delta_{m}(A), \quad A \in \mathfrak{L}, m \in \mathbb{Z}
$$

Obsérvese que $\gamma_{m}(A)$ está en el anillo $\mathcal{D}_{0}$ para cada $m$ y $\delta_{m}(A)=S^{m} \gamma_{m}(A)$. Por lo tanto,

$$
A=\sum_{k \geq i(A)} S^{k} \gamma_{k}(A), \quad A \in \mathfrak{L}
$$

Decimos que (5.8) es la representación por diagonales de la matriz A. Por lo tanto, el anillo de matrices $\mathfrak{L}$ es el conjunto de matrices de la forma

$$
A=\sum_{k \geq i(A)} S^{k} U_{k}, \quad U_{k} \in \mathcal{D}_{0}, U_{i(A)} \neq 0
$$

junto con la matriz cero. Esta representación de los elementos de $\mathfrak{L}$ parece una serie de Laurent donde $S$ desempeña el papel de un símbolo indeterminado (no es una variable, sólo nos ayuda a representar las series) y las matrices $U_{k}$ son los coeficientes, pero en general $S$ no conmuta con las matrices $U_{k}$. Obsérvese el siguiente ejemplo sencillo sobre representación por diagonales de una matriz $A$ doblemente infinita.

EJEMPlo 5.5 Sea $A \in \mathfrak{L}$ con $i(A)=-1$, así su representación por diagonales es como sigue

$$
\begin{gathered}
A=\left(\begin{array}{ccccccc}
\ddots & \vdots & \vdots & \vdots & \vdots & \\
\cdots & a_{-2,-2} & a_{-2,-1} & 0 & 0 & \cdots \\
\cdots & a_{-1,-2} & a_{-1,-1} & a_{-1,0} & 0 & \cdots \\
\cdots & a_{0,-2} & a_{0,-1} & a_{0,0} & a_{0,1} & \cdots \\
\cdots & a_{1,-2} & a_{1,-1} & a_{1,0} & a_{1,1} & \cdots \\
& \vdots & \vdots & \vdots & \vdots & \ddots
\end{array}\right) \\
\\
\end{gathered}
$$


A continuación se encontrará una expresión para la multiplicación en $\mathfrak{L}$ usando la representación por diagonales.

Teorema 5.7 Sean $A$ y $B$ elementos de $\mathfrak{L}$

$$
\begin{array}{ll}
A=\sum_{k \geq i(A)} S^{k} U_{k}, & U_{k} \in \mathcal{D}_{0}, U_{i(A)} \neq 0, \\
B=\sum_{k \geq i(B)} S^{k} V_{k}, & V_{k} \in \mathcal{D}_{0}, V_{i(B)} \neq 0 .
\end{array}
$$

Entonces

$$
A B=\sum_{n \geq i(A)+i(B)} S^{n} \sum_{k=i(B)}^{n-i(A)} U_{n-k}^{[-k]} V_{k} .
$$

Demostración. Por la Proposición 5.6, parte 7, se tiene

$$
S^{n-k} U_{n-k} S^{k} V_{k}=S^{n} U_{n-k}^{[-k]} V_{k}, \quad n, k \in \mathbb{Z},
$$

donde $U_{n-k}^{[-k]}$ es el desplazamiento diagonal de $U_{n-k}$ por $-k$ pasos y satisface

$$
\left(U_{n-k}^{[-k]}\right)_{j, j}=\left(U_{n-k}\right)_{j+k, j+k}
$$

para $j \in \mathbb{Z}$. Los límites de la suma sobre $k$ en (5.10) se obtienen de $k \geq i(B)$ y $n-k \geq i(A)$.

El anillo $\mathcal{D}_{0}$, el cual es un subanillo de $\mathfrak{L}$, es isomorfo al conjunto de funciones de $\mathbb{Z}$ al anillo $R$, equipado con sus operaciones naturales de adición y multiplicación de funciones. Nótese que este anillo contiene muchos divisores de cero y que es conmutativo sólo cuando $R$ es conmutativo.

Del Teorema 5.7 se observa que $\mathfrak{L}$ es una modificación no conmutativa del anillo de series formales de Laurent con coeficientes que son funciones de $\mathbb{Z}$ a $R$. Considerando a $S$ como un tipo de símbolo indeterminado (no es una variable, sino que nos ayuda a representar las series formales de Laurent), en general, $S$ no conmuta con los coeficientes $U_{k}$, los cuales están en $\mathcal{D}_{0}$, es lo que hace que los desplazamientos diagonales aparezcan en la fórmula de multiplicación (5.10).

La representación en serie de Laurent proporciona de inmediato una condición suficiente para la invertibilidad en $\mathfrak{L}$.

Proposición 5.8 Sea $A=\sum_{k \geq i(A)} S^{k} U_{k}$, donde las matrices $U_{k}$ están en $\mathcal{D}_{0}$, y supongamos que $U_{i(A)}$ es invertible. Entonces $A$ es invertible en $\mathfrak{L}$.

Demostración. Sea

$$
B=\sum_{k \geq-i(A)} S^{k} V_{k}, \quad V_{k} \in \mathcal{D}_{0} .
$$

Se mostrará que a partir de la ecuación $A B=I$ es posible obtener todas las matrices $V_{k}$ de forma recursiva. Por el Teorema 5.7 se tiene

$$
A B=\sum_{n \geq 0} S^{n} \sum_{k=-i(A)}^{n-i(A)} U_{n-k}^{[-k]} V_{k} .
$$


Si $A B=I$ entonces el sumando correspondiente a $n=0$ en la suma anterior debe ser igual a la matriz identidad $I$. Es decir, $U_{i(A)}^{[i(A)]} V_{-i(A)}=I$. Además $V_{-i(A)}$ debe ser igual a la inversa de $U_{i(A)}^{[i(A)]}$ la cual existe ya que $U_{i(A)}$ es invertible por hipótesis. Para $n>0$ el coeficiente de $S^{n}$ en la expresión para $A B$ debe ser cero, por lo tanto,

$$
U_{i(A)}^{[i(A)-n]} V_{n-i(A)}=-\sum_{k=-i(A)}^{n-1-i(A)} U_{n-k}^{[-k]} V_{k} .
$$

Como $U_{i}(A)$ es invertible, también lo son sus desplazamientos diagonales, y luego de la ecuación anterior se obtiene $V_{n-i(A)}$ en términos de $V_{k}$, con $-i(A) \leq k \leq n-1-i(A)$, y desplazamientos diagonales de algunas matrices de coeficientes de $A$. Por lo tanto, todas las matrices de coeficientes diagonales $V_{k}$ se determinan de manera única de forma recursiva. Usando la sustitución directa es fácil encontrar una expresión explícita, pero bastante larga, para las matrices $V_{k}$. Por ejemplo,

$$
V_{-i(A)+1}=-V_{-i(A)} U_{i(A)+1}^{[i(A)]} V_{-i(A)},
$$

y

$$
V_{-i(A)+2}=V_{-i(A)} U_{i(A)+1}^{[i(A)]} V_{-i(A)} U_{i(A)+1}^{[i(A)]} V_{-i(A)}-V_{-i(A)} U_{i(A)+2}^{[i(A)]} V_{-i(A)} .
$$

Si en lugar de la ecuación $A B=I$ se considera $B A=I$, usando inducción y algunos cálculos sencillos, es posible mostrar que los coeficientes de la matriz $B$ obtenidos en este caso coinciden con los correspondientes coeficientes de la matriz $B$ obtenida anteriormente. Alternativamente, se puede demostrar que la matriz $B$ obtenida anteriormente satisface $B A=I$, por lo tanto, $B$ es la única inversa bilateral de $A$. Esto completa la demostración.

Para las series de Laurent en forma usual, el resultado análogo a la Proposición 5.8 a menudo se demuestra mediante el uso de series geométricas. Tal método de prueba también funciona en nuestro contexto, como se mostrará a continuación. Es decir, otra forma de demostrar la Proposición 5.8 es la siguiente:

Demostración. Sea $B=S^{-i(A)} A U_{i(A)}^{-1}$. Entonces

$$
B=\sum_{n \geq 0} S^{n} U_{n+i(A)} U_{i(A)}^{-1}
$$

y por lo tanto $B=I+C$ donde $C=\sum_{n \geq 1} S^{n} V_{n}$ y las matrices coeficientes $V_{n}=U_{n+i(A)} U_{i(A)}^{-1}$ están en $\mathcal{D}_{0}$. Las potencias positivas de $C$ tienen índices crecientes, es decir, las potencias de $C$ están bajando de diagonal. Por lo tanto, los elementos $f_{i j}$ de la serie geométrica

$$
F=\sum_{n \geq 0}(-C)^{n}
$$

son $f_{i j}=(C)_{i j}+\left(C^{2}\right)_{i j}+\left(C^{3}\right)_{i j}+\cdots+\left(C^{m}\right)_{i j}$. En cada diagonal sólo contribuyen a la suma las potencias anteriores desde la diagonal 1 hasta la diagonal $m$, entonces para cada $m$ positiva sólo los primeros $m$-términos de esta suma contribuyen a esa diagonal, ya que para los que siguen (las potencias mayores a $m$ ), las entradas en esa diagonal son cero. Por lo tanto, $F$ está bien definida y es claramente una inversa bilateral de $B$ y además es la única inversa bilateral de $B$. Ahora bien, dado que $A=S^{i(A)} B U_{i(A)}$, es fácil ver que $U_{i(A)}^{-1} F S^{-i(A)}$ es el único inverso bilateral de $A$. 
La condición para la invertibilidad de la Proposición 5.8 no es necesaria. Considérese el siguiente ejemplo sencillo.

EJEMPLO 5.6 Sea $R$ un anillo de matrices $2 \times 2$ sobre $\mathbb{R}$ y sean

$$
a=\left(\begin{array}{cc}
0 & 0 \\
1 & 0
\end{array}\right), \quad b=\left(\begin{array}{ll}
0 & 1 \\
0 & 0
\end{array}\right) .
$$

Los elementos $a$ y $b$ son divisores de cero, ya que $a^{2}=b^{2}=0$, y $a b+b a=e$, la identidad en $R$. Sea $A=I a+S b$. Obsérvese que $A$ es una matriz de Toeplitz. Un simple cálculo muestra que $I b+S^{-1} a$ es el inverso de $A$. La descomposición en bloques de las matrices infinitas explica el porqué funciona este ejemplo y también el porqué no es fácil encontrar condiciones necesarias para la invertibilidad en $\mathfrak{L}$, es decir, el problema de caracterizar los elementos invertibles en las matrices doblemente infinitas, es un problema interesante y no trivial.

\subsection{Algunas propiedades del anillo $\mathfrak{L}$}

En esta sección se usa la representación diagonal de los elementos de $\mathfrak{L}$ con el propósito de obtener algunas propiedades relacionadas con similaridad y conmutatividad.

TeOrema 5.9 Sea

$$
A=\sum_{k \geq i(A)} S^{k} U_{k}, \quad U_{k} \in \mathcal{D}_{0}
$$

y suponemos que $i(A) \neq 0$ y cada entrada de $U_{i(A)}$ es un elemento invertible del centro de $R$. Entonces existe una matriz invertible $P$ de índice cero tal que

$$
P^{-1} A P=S^{i(A)} U_{i(A)} .
$$

Demostración. Obsérvese que $U_{i(A)}$ es un elemento invertible del centro del anillo $\mathcal{D}_{0}$. Sea

$$
P=\sum_{k \geq 0} S^{k} V_{k}
$$

donde las matrices de coeficientes $V_{k}$ están en $\mathcal{D}_{0}$. Considérese la ecuación $A P=P S^{i(A)} U_{i(A)}$. Se encontrarán matrices $V_{k}$ que satisfacen esta ecuación y tal que $P$ es invertible. Por la fórmula de multiplicación (5.10) la ecuación $A P=P S^{i(A)} U_{i(A)}$ se convierte en

$$
\sum_{n \geq i(A)} S^{n} \sum_{k=0}^{n-i(A)} U_{n-k}^{[-k]} V_{k}=\sum_{r \geq 0} S^{r+i(A)} V_{k}^{[-i(A)]} U_{i(A)} .
$$

Al igualar los coeficientes correspondientes de $S^{r+i(A)}$ en ambos lados de la ecuación anterior se obtiene

$$
\sum_{k=0}^{r} U_{r-k+i(A)}^{[-k]} V_{k}=V_{r}^{[-i(A)]} U_{i(A)} .
$$

Cuando $r=0$ se tiene $U_{i(A)} V_{0}=V_{0}^{[-i(A)]} U_{i(A)}$, que se reduce a $V_{0}=V_{0}^{[-i(A)]}$, ya que $U_{i(A)}$ es un elemento invertible del centro de $\mathcal{D}_{0}$. Por tanto, cada elección arbitraria de $|i(A)|$ elementos 
invertibles de $R$ produce una solución para $V_{0}$. Una solución obvia es $V_{0}=I$. Si $r \geq 1$ entonces el lado izquierdo de la ecuación (5.11) se puede escribir como

$$
U_{i(A)}^{[-r]} V_{r}+\sum_{k=0}^{r-1} U_{r-k+i(A)}^{[-k]} V_{k}=V_{r}^{[-i(A)]} U_{i(A)} .
$$

Si $V_{0}, V_{1}, \ldots, V_{r-1}$ han sido determinados entonces la ecuación anterior puede resolverse para $V_{r} \mathrm{y}$ se obtiene una expresión que depende del desplazamiento diagonal $V_{r}^{[-i(A)]}$, los $V_{k}$ ya conocidos y algunas de las matrices de coeficientes $U_{k}$ de $A$. Obsérvese que $P$ no es única, ya que se pueden elegir $|i(A)|$ entradas arbitrarias de $V_{k}$ en cada paso.

Si la matriz $U_{i(A)}$ del Teorema 5.9 es igual a la matriz identidad $I$, entonces

$$
A=P S^{i(A)} P^{-1},
$$

y por lo tanto la matriz $B=P S P^{-1}$ satisface $B^{i(A)}=A$. Es decir, $B$ es una raíz de orden $i(A)$ de $A$. Nótese que $B$ depende de $P$ y $P$ no es única.

Para matrices de índice cero se obtiene el siguiente resultado.

Teorema 5.10 Sea

$$
A=\sum_{k \geq 0} S^{k} U_{k}, \quad U_{k} \in \mathcal{D}_{0}
$$

y suponemos que $U_{0}$ es un elemento del centro de $\mathcal{D}_{0}$ y $U_{0}-U_{0}^{[m]}$ es invertible para cada entero $m$ distinto de cero. Entonces existe una matriz invertible $P$ de índice cero tal que

$$
P^{-1} A P=U_{0} .
$$

Demostración. Sea $P=\sum_{k \geq 0} S^{k} V_{k}$ con $V_{0}=I$. La ecuación $A P=P U_{0}$ es equivalente a

$$
\sum_{n \geq 0} S^{n} \sum_{k=0}^{n} U_{n-k}^{[-k]} V_{k}=\sum_{n \geq 0} S^{n} V_{n} U_{0} .
$$

Igualando los coeficientes de $S^{n}$ en ambos lados de la ecuación anterior se obtiene

$$
U_{0}^{[-n]} V_{n}+\sum_{k=0}^{n-1} U_{n-k}^{[-k]} V_{k}=V_{n} U_{0}=U_{0} V_{n} .
$$

Esta ecuación es trivial si $n=0$ y por la hipótesis, para $n \geq 1$ se puede escribir como

$$
V_{n}=\left(U_{0}-U_{0}^{[-n]}\right)^{-1} \sum_{k=0}^{n-1} U_{n-k}^{[-k]} V_{k},
$$

y por lo tanto las matrices $V_{k}$ se pueden calcular de forma recursiva.

Nótese que la matriz diagonal $U_{0}$ del Teorema 5.10 no es necesariamente invertible. Puede tener una entrada igual a cero en la diagonal de índice cero. Por ejemplo, $\left(U_{0}\right)_{k, k}=k$ para $k$ en $\mathbb{Z}$, con $R=\mathbb{R}$.

Teorema 5.11 Sea $U$ un elemento del centro del anillo $\mathcal{D}_{0}$ tal que para cada entero $m$ distinto de cero la matriz $U-U^{[m]}$ no tiene divisores de cero en su diagonal de índice cero. Entonces, si $B$ es un elemento de $\mathfrak{L}$ que conmuta con $U$ se debe tener que $B \in \mathcal{D}_{0}$. 
Demostración. Sea $B=\sum_{k \geq i(B)} S^{k} V_{k}$, donde las $V_{k}$ están en $\mathcal{D}_{0}$. Si $U B=B U$ entonces

$$
\sum_{k \geq i(B)} S^{k} U^{[-k]} V_{k}=\sum_{k \geq i(B)} S^{k} V_{k} U
$$

Por ende, igualando los coeficientes de ambos lados de la igualdad anterior se tiene,

$$
U^{[-k]} V_{k}=V_{k} U=U V_{k} \quad \text { para } \quad k \geq i(B) .
$$

Si $k \neq 0$ se obtiene $\left(U-U^{[-k]}\right) V_{k}=0$ y entonces $V_{k}=0$, ya que por hipótesis $U-U^{[-k]}$ no tiene divisores de cero en la diagonal de índice cero. Por lo tanto, $B=V_{0}$ y está en $\mathcal{D}_{0}$.

El teorema siguiente es una generalización de la Proposición 5.6, parte 2.

Teorema 5.12 Sean $m$ un entero distinto de cero y $U$ un elemento invertible de $\mathcal{D}_{0}$. Sea $B=$ $\sum_{k>i(B)} S^{k} V_{k}$, donde los $V_{k} \in \mathcal{D}_{0}$. Si $B$ conmuta con $S^{m} U$ entonces

$$
V_{k}^{[-m]}=U^{[-k]} V_{k} U^{-1} \quad \text { para } \quad k \geq i(B) .
$$

Demostración. Si $B S^{m} U=S^{m} U B$ entonces

$$
\sum_{k \geq i(B)} S^{k} V_{k} S^{m} U=\sum_{k \geq i(B)} S^{m} U S^{k} V_{k}
$$

o bien

$$
\sum_{k \geq i(B)} S^{k+m} V_{k}^{[-m]} U=\sum_{k \geq i(B)} S^{m+k} U^{[-k]} V_{k} .
$$

Comparando los coeficientes de la igualdad anterior se tiene,

$$
S^{k+m} V_{k}^{[-m]} U=S^{m+k} U^{[-k]} V_{k}, \quad k \geq i(B),
$$

y esto completa la demostración.

La ecuación $V_{k}^{[-m]}=U^{[-k]} V_{k} U^{-1}$ significa que

$$
\left(V_{k}\right)_{j+m, j+m}=(U)_{j+k, j+k}\left(V_{k}\right)_{j, j}\left(U^{-1}\right)_{j, j}, \quad j \in \mathbb{Z} .
$$

Cuando $U$ es la matriz identidad, esta ecuación se reduce a $\left(V_{k}\right)_{j+m, j+m}=\left(V_{k}\right)_{j, j}$ entonces las diagonales de $B$ son periódicas, y $B$ es una matriz de Toeplitz por bloques. Por lo tanto, en el caso general, cada diagonal de $B$ está determinada por cualesquiera $m$ entradas consecutivas de la ecuación anterior y se puede considerar a $B$ como algún tipo de matriz Toeplitz por bloques generalizada.

A continuación se demostrará que los elementos de $\mathfrak{L}$ también se pueden expresar como series de Laurent con un desplazamiento ponderado en lugar del desplazamiento $S$.

Sea $W$ un elemento invertible de $\mathcal{D}_{0}$. Definimos el desplazamiento ponderado $T$ con peso $W$ por

$$
T=S W
$$

Entonces $T^{2}=S W S W=S^{2} W^{[-1]} W$ y $T^{3}=S^{3} W^{[-2]} W^{[-1]} W$. Es posible encontrar una expresión para $T^{k}$ de la siguiente forma: 
Se define $W_{(0)}=I$ y para $k \geq 1$ se define

$$
W_{(k)}=W^{[-k+1]} W^{[-k+2]} \cdots W^{[-2]} W^{[-1]} W .
$$

Ésta es llamada potencia factorial ascendente de $W$. Así se tiene $T^{k}=S^{k} W_{(k)}$ para $k \geq 0$. Por lo tanto, para $k \geq 1$ se tiene

$$
T^{-k}=W_{(k)}^{-1} S^{-k}=S^{-k} S^{k} W_{(k)}^{-1} S^{-k}=S^{-k}\left(W_{(k)}^{-1}\right)^{[k]}
$$

Se define $W_{(-k)}=\left(W_{(k)}^{-1}\right)^{[k]}$ para $k \geq 1$. Con esta notación se obtiene

$$
T^{k}=S^{k} W_{(k)} \quad \text { para } \quad k \in \mathbb{Z} .
$$

Teorema 5.13 Sea $A=\sum_{k \geq i(A)} S^{k} U_{k}$, con $U_{k} \in \mathcal{D}_{0}$. Sea $W$ un elemento invertible de $\mathcal{D}_{0}$ y $T=$ $S W$. Entonces $A=\sum_{k \geq i(A)} T^{k} V_{k}$, donde $V_{k}=W_{(k)}^{-1} U_{k}$ para $k \geq i(A)$.

Demostración. $\quad T^{k} V_{k}=T^{k} W_{(k)}^{-1} U_{k}=S^{k} U_{k}$ para $k \geq i(A)$.

A continuación se consideran algunos mapeos útiles en el anillo $\mathfrak{L}$ relacionados con conmutadores.

Para cada $A$ en $\mathfrak{L}$, se define la derivada de Pincherle de $A$ por $A^{\prime}=A S-S A$. Es fácil mostrar que la derivada de Pincherle es una derivación en $\mathfrak{L}$, es decir,

$$
(A B)^{\prime}=A B^{\prime}+A^{\prime} B, \quad A, B, \in \mathfrak{L} .
$$

Obsérvese que la derivada de Pincherle de cualquier matriz de Toeplitz es igual a cero.

Si $A=\sum_{k \geq i(A)} S^{k} U_{k}$ entonces un cálculo simple da

$$
A^{\prime}=\sum_{k \geq i(A)} S^{k+1}\left(U_{k}^{[-1]}-U_{k}\right) .
$$

Nótese que la entrada $(j, j)$ de $U_{k}^{[-1]}-U_{k}$ es igual a $\left(U_{k}\right)_{j+1, j+1}-\left(U_{k}\right)_{j, j}$ para $j$ en $\mathbb{Z}$.

Ahora, se denotará la matriz diferenciación $D$ por $D=S^{-1} N$, donde $N \in \mathcal{D}_{0}$ y $(N)_{k, k}=k$. La derivada de Pincherle de $D$ es

$$
D^{\prime}=D S-S D=S^{-1} N S-N=N^{[-1]}-N=I .
$$

Usando inducción se puede ver que $\left(D^{m}\right)^{\prime}=m D^{m-1}$ para $m \geq 1$.

La derivada de Pincherle de segundo tipo se define por

$$
A^{\circ}=D A-A D, \quad A \in \mathfrak{L} .
$$

Ésta es una derivación sobre $\mathfrak{L}$ y es claro que $S^{\circ}=I$ y $\left(S^{k}\right)^{\circ}=k S^{k-1}$ para $k$ en $\mathbb{Z}$. Un simple cálculo da

$$
\left(S^{k} U\right)^{\circ}=S^{k-1}\left(N U-U^{[1]} N+k U\right), \quad U \in \mathcal{D}_{0}
$$


y entonces, para $A=\sum_{k \geq i(A)} S^{k} U_{k}$, se tiene

$$
A^{\circ}=\sum_{k \geq i(A)} S^{k-1}\left(N\left(U_{k}-U_{k}^{[1]}\right)+k U_{k}\right) .
$$

Obsérvese que ambos tipos de derivadas de $A \in \mathcal{L}$ involucran diferencias $U_{k}-U_{k}^{[1]}$ de las matrices de coeficientes de $A$.

Se ha demostrado que la representación diagonal es una herramienta útil para tratar los elementos de $\mathfrak{L}$, para cálculos y también para derivar propiedades generales. 
El estudio y desarrollo de familias de sucesiones de polinomios ortogonales utilizando matrices infinitas tal y como se mencionó en la Introducción de la presente Tesis, L. Verde mostró en [34] que una parte considerable de la teoría general se puede obtener usando herramientas puramente algebraicas del álgebra lineal en el contexto de una álgebra de matrices infinitas.

Siguiendo con el trabajo iniciado, estudiamos las sucesiones de momentos de polinomios ortogonales $q$-clásicos obteniendo una relación de recurrencia de tres términos con coeficientes variables que dependen de los coeficientes de la ecuación en $q$-diferencias satisfecha por los polinomios. Consideramos los casos en los cuales la relación de recurrencia se simplifica y obtuvimos algunas familias de polinomios ortogonales $q$-clásicos que tienen fórmulas explícitas simples para los momentos, expresadas en términos de los parámetros en la ecuación en $q$-diferencias.

Se construyeron varios ejemplos de familias de polinomios ortogonales $q$-clásicos que tienen sucesiones simples de momentos. Con ayuda de cinco parámetros se encontraron fórmulas explícitas para los momentos y los coeficientes de la relación de recurrencia. Observamos que dando un valor particular a ciertos parámetros, la matriz $C$ se convierte en bidiagonal y entonces esto permite obtener una relación de recurrencia a dos términos. Así, dando algunos valores apropiados a los parámetros se obtuvieron familias de polinomios $q$-ortogonales tales como: los polinomios $q$-Krawtchouk no estándar, y que no han sido considerados en la literatura de polinomios ortogonales, los polinomios $q$-Bessel, los polinomios $q$-Laguerre/Wall pequeños y los polinomios $q$-Jacobi grandes.

Por otro lado, se ha demostrado que la representación diagonal es una herramienta útil para tratar los elementos del conjunto de matrices doblemente infinitas, $\mathfrak{L}$, para cálculos y también para derivar propiedades generales. También es importante mencionar que el problema de caracterizar los elementos invertibles en las matrices doblemente infinitas, es un problema interesante y no trivial, incluso cuando se este haciendo sobre un campo. Se presentaron algunos ejemplos de lo que se puede hacer con la representación diagonal, pero está claro que muchas otras propiedades algebraicas de las matrices infinitas se pueden obtener con nuestros métodos. Nótese que el anillo $R$ puede ser, por ejemplo, el álgebra de $n \times n$ matrices sobre algún campo.

El anillo de matrices doblemente infinitas $\mathfrak{L}$, ha sido poco estudiado, por ello se quiere primero entender los casos más simple, por ejemplo, cuando pasamos sobre un campo o un anillo de matrices tales como matrices $2 \times 2$, pues esto ya es suficiente para tener problemas interesantes, ya que la descomposición por bloques es una herramienta poderosa en el estudio de matrices doblemente infinitas. Es por esta razón que pensamos en $R$ como un anillo (más o menos) arbitrario, porque un caso interesante es que éste sea un anillo de matrices, para poder trabajar en matrices por bloques. Además, cada una de las filas de las matrices doblemente infinitas está representando series de Laurent invertidas ortogonales (puesto que tenemos un número finito de términos con potencias 
positivas), y cuando tenemos matrices doblemente infinitas en banda se tienen polinomios ortogonales de Laurent (ya que se tiene un número finito de términos distintos de cero en cada fila). Para conocer más sobre polinomios ortogonales de Laurent, véase [10] y sus referencias.

En el anillo $\mathfrak{L}$, es posible estudiar polinomios ortogonales de Laurent, grupos de Riordan generalizados, fórmulas de inversión de Lagrange y cálculo umbral. Hasta aquí, no se ha considerado la dualidad inducida por el reflejo de las matrices con respecto a la anti-diagonal $n+k=-1$. La importancia de esa dualidad se ha demostrado en [6], [32] y [33].

Finalmente, la extensión al caso de multi-matrices (índices en $\mathbb{Z}^{d}$ ) es un tema para futuras investigaciones. Véase [32], donde se estudió la inversión de Lagrange en varias variables en un anillo de multi-matrices. 
[1] R. Álvarez-Nodarse, On characterizations of classical polynomials, J. Comput. Appl. Math. 196 (2006) 320-337.

[2] W.A. Al Salam, T.S. Chihara, Another characterization of the classical orthogonal polynomials, SIAM J.Math. Anal. 3 (1972) 65-70.

[3] R. Álvarez-Nodarse, R. Sevinik Adigüzel, H. Taseli, On the orthogonality of q-classical polynomials of the Hahn class, SIGMA Symmetry Integrability Geom. Methods Appl. 8 (2012), Paper 042, 30 pp.

[4] M.I. Arenas-Herrera, L. Verde-Star, Classical q-orthogonal polynomials that have simple sequences of moments. (Este artículo se encuentra en preparación).

[5] M.I. Arenas-Herrera, L. Verde-Star, Representation of doubly infinite matrices as noncommutative Laurent series, Special Matrices, Volume 5 (2017) 250-257.

[6] M. Barnabei, A. Brini, G. Nicoletti, Recursive matrices and Umbral Calculus, J. of Algebra, 75 (1982) 546-573.

[7] S. Bochner. Über Sturm-Liouvillesche polynomsysteme, Math. Zeit. 29 (1929), 730-736.

[8] T. S. Chihara, An introduction to orthogonal polynomials, Gordon and Breach, N. Y. 1978.

[9] A.J. Durán, Functions with given moments and weight functions for orthogonal polynomials, Rocky Mountain J. Math. 23 (1993) 87-103.

[10] E. Hendriksen, H. van Rossum, Orthogonal Laurent polynomials, Nederl. Akad. Wetensch. Proc. Ser. A, 89 (1986) 17-36.

[11] P. Henrici, Applied and computational Complex Analysis, Vol. I, J. Wiley, N. Y. 1974.

[12] E. Jabotinsky, Analytic iteration, Trns. Amer. Math. Soc.108(1963) 457-477.

[13] L. Khériji, P. Maroni, The Hq-Classical orthogonal polynomials, Acta Aplicandae Math. 71 (2002) 49-115. 
[14] R. Koekoek, P.A. Lesky, R.F. Swarttouw, Hypergeometric orthogonal polynomials and their q-analogues, Springer-Verlag, Berlin, Heidelberg, 2010.

[15] G. Köthe, O. Toeplitz, Theorie der halbfiniten unendlichen Matrizen, J. Reine Angew. Math. 165 (1931) 116-127.

[16] T. H. Koornwinder, Orthogonal polynomials in connection with quantum groups. In: Orthogonal Polynomials: Theory and Practice (ed. P. Nevai), Kluwer, Dordrecht, 1990, 257-292.

[17] K.H. Kwon, D.W. Lee, S.B. Park, B.H. Yoo, Hahn class orthogonal polynomials, Kyungpook Math. J. 38 (1998) 259-281.

[18] A. F. Loureiro, New results on the Bochner condition about classical orthogonal polynomials, J. Math. Anal. Appl. 364 (2010) 307-323.

[19] A. Luzón, D. Merlini, M.A. Morón, R. Sprugnoli, Identities induced by Riordan arrays, Linear Algebra Appl. 436 (2012) 631-647.

[20] A. Luzón, M.A. Morón, J.L. Ramírez, Double parameter recurrences for polynomials in biinfinite Riordan matrices and some derived identities, Linear Algebra Appl. 511 (2016) 237-258.

[21] P. Maroni, Variations around classical orthogonal polynomials. Connected problems, J. Comput. Appl. Math. 48 (1993) 133-155.

[22] P. Maroni, M. Mejri, The I(q,w) classical orthogonal polynomials, Appl. Numer. Math. 43 (2002) 423-458.

[23] J.C. Medem, F. Marcellán, q-Classical polynomials: a very classical approach, Electron. Trans. Numer. Anal. 9 (1999) 112-127.

[24] J.C. Medem, R. Álvarez-Nodarse, F. Marcellán, On the q-polynomials: a distributional study, J. Comp. Appl. Math. 135 (2001) 157.

[25] P. Njionou Sadjang, Moments of classical orthogonal polynomials, PhD thesis, Universität Kassel, 2013.

[26] P. Njionou Sadjang, W. Koepf, M. Foupouagnigni, On moments of classical orthogonal polynomials, J. Math. Anal. Appl. 424 (2015) 122-151.

[27] E. J. Routh, On some properties of certain solutions of a differential equation of the second order, Proc London Math Soc., 16 (1885), 245-261.

[28] L. J. Slater, Generalized Hypergeometric Functions, Cambridge University Press, 1966.

[29] T. J. Stieltjes. Recherches sur les fractions continues, Annales de la faculté des sciences de Toulouse Sér. 6 4(2) (1995), 36-75.

[30] G. Szegö, Orthogonal polynomials, Amer. Math. Soc., N. Y., 1939.

[31] C.V.M. van der Mee, S. Seatzu, G. Rodriguez, Spectral factorization of bi-infinite multi-index block Toeplitz matrices, Linear Algebra Appl. 343/344 (2002) 355-380.

[32] L. Verde-Star, Dual operators and Lagrange inversion in several variables, Adv. in Math. 58 (1985) 89-108. 
[33] L. Verde-Star, Groups of generalized Pascal matrices, Linear Algebra Appl. 382 (2004) 179-194.

[34] L. Verde-Star, Characterization and construction of classical orthogonal polynomials using a matrix approach, Linear Algebra Appl. 438 (2013) 3635-3648.

[35] L. Verde-Star, Polynomial sequences generated by infinite Hessenberg matrices, Special Matrices $2017 ; 5: 64-72$.

[36] L. Verde-Star, Recurrence coefficients and difference equations of classical discrete orthogonal and q-orthogonal polynomial sequences, Linear Algebra Appl. 440 (2014) 293-306. 


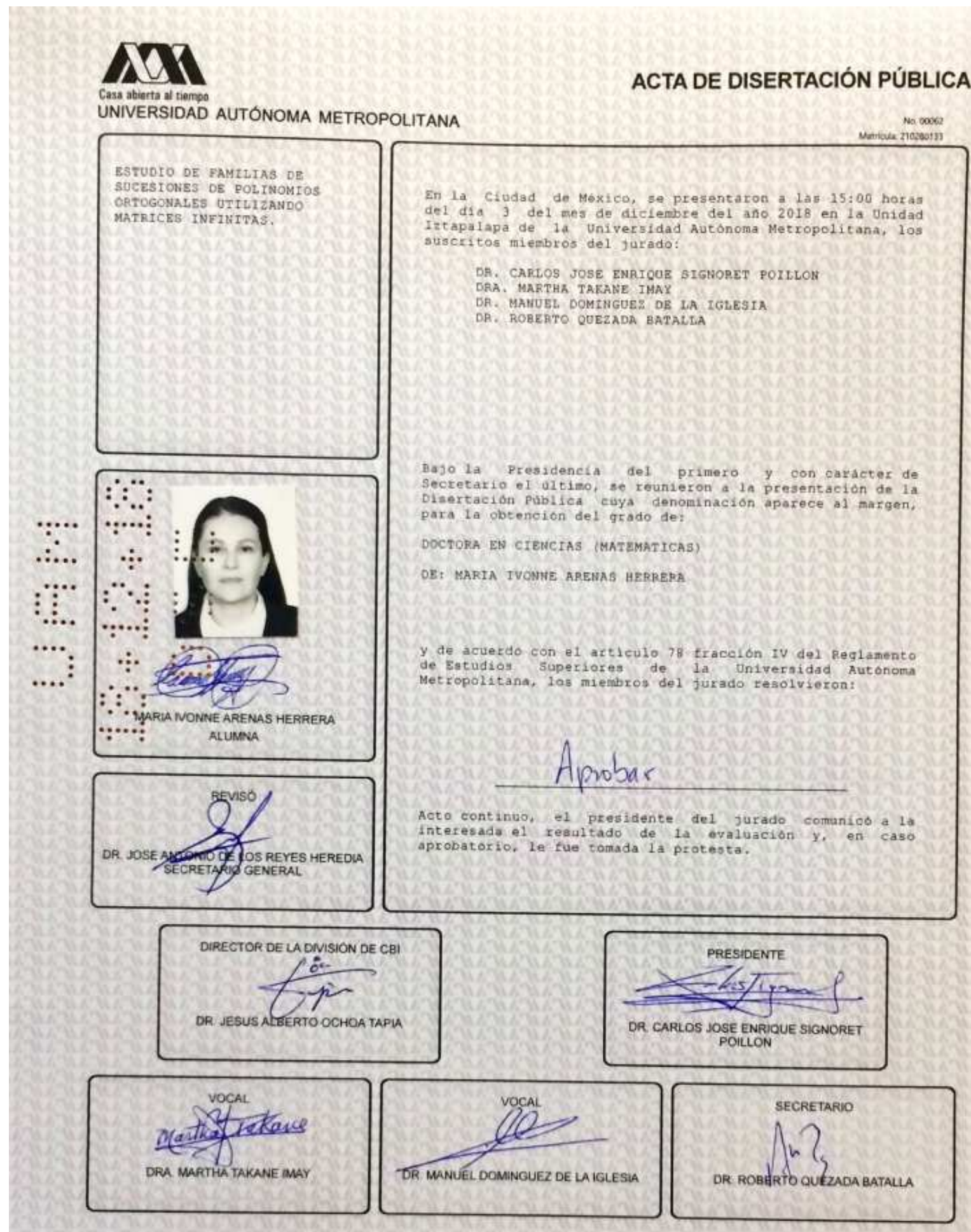

